\title{
La scultura in funzione architettonica a Costantinopoli tra V e VI secolo: aspetti tecnici, tipologici e stilistici ${ }^{*}$
}

\author{
In ricordo di Fernanda de' Maffei \\ nel centesimo anniversario della nascita
}

\begin{abstract}
Data la complessità e l'ampiezza dell'argomento, s'intende proporre solo alcune riflessioni su quegli 'episodi' e quegli esempi, molti ben conosciuti e più volte analizzati, altri invece meno noti, i quali potranno meglio illustrare i momenti più significativi dello sviluppo della scultura architettonica costantinopolitana lungo un percorso che conduce alle straordinarie novità che si manifestano nei primi decenni del VI secolo. È un campo d'indagine assai vasto, che ha alimentato e continua ad alimentare una letteratura critica altrettanto ampia, ricca di saggi e contributi, con approcci e chiavi di lettura variamente declinati, ma che alla somma dei fatti, si rivelano proficuamente complementari.
\end{abstract}

Nell'ambito di un contesto documentario disomogeneo e frammentario, con sporadiche attestazioni tra loro del tutto scollegate e di problematico inquadramento cronologico, la prima concreta testimonianza relativa alla scultura architettonica costantinopolitana è rappresentata dagli elementi dell'imponente arco che si ergeva lungo il percorso della Mese, sul limite occidentale del Foro di Teodosio I, il Forum Tauri, inaugurato, ricordo, nel 393, senz'altro il complesso monumentale più prestigioso e rappresentativo del programma di sviluppo urbanistico della neocapitale promosso dall'imperatore Teodosio I (379-395). ${ }^{1}$

Malgrado l'indubbia importanza, questa notevole collezione di materiali, tutti di marmo proconnesio, venuti alla luce in momenti diversi all'interno del settecentesco complesso del

\footnotetext{
* Claudia Barsanti è scomparsa nel settembre del 2017. La correzione delle b ozze della parte dell'articolo da lei firmata è stata eseguita, con minimi aggiornamenti bibliografici, da Andrea Paribeni.

${ }^{1}$ Per la topografia e l'ampiezza del complesso forense che si estendeva sulla piattaforma della III collina del promontorio costantinopolitano, per i suoi monumenti e il suo arredo scultoreo, cfr. Müller-Wiener 1977, 258-265; Faedo 1982; Barsanti 1995; Bauer 1996, 187-203; Berger 1996; Faedo 1997; Faedo 1998; Bassett 2004, 82-83, 93, 208-212; Barsanti in c.d.s.
} 
Simkeş $\operatorname{Han}^{2}$ a partire dal $1920,{ }^{3}$ quindi nel $1928^{4}$ e poi nel $1957,{ }^{5}$ in occasione di scavi per lo più effettuati in concomitanza dei grandi progetti urbanistici finalizzati all'ammodernamento dell'area di Beyazit, ${ }^{6}$ e d'allora sistemati nelle aiuole circostanti i basamenti del fornice centrale dell'arco, ${ }^{7}$ sul marciapiede meridionale della trafficatissima Ordu Caddesi (FIG. 1), non è mai stata interamente catalogata. ${ }^{8} \mathrm{Di}$ conseguenza sono rimasti senza risposta molti quesiti e alcuni dubbi relativi alla travagliata storia del monumento ed anche nei riguardi della ricostruzione proposta da Rudolf Naumann. ${ }^{9}$

Il disegno, come pure le membrature architettoniche dell'ordine corinzio dell'arco, palesano stretti legami con i modelli classici di tradizione microasiatica, che però, al momento, non sembrano offrire alcun riscontro oggettivo per le singolarissime colonne modellate a guisa di nodose clave impugnate da una gigantesca mano, di cui è stato più volte sottolineato lo straordinario carico di valenza propagandistica, volta ad esaltare con grande impatto visivo le virtù eroiche dell'imperium di Teodosio I. $^{10}$

Allo stato attuale la ricognizione dei materiali è piuttosto disagevole, complicata dal parziale interro e dal 'movimento' dei marmi che vengono di continuo spostati e disordinatamente ammassati, ${ }^{11}$ tant'è vero che è sempre più difficile ispezionarli, specie $\mathrm{i}$ grandi capitelli corinzi, senz'altro gli elementi più noti del monumento. Gli esemplari superstiti sono quattro, ${ }^{12}$ tutti in condizioni più o meno frammentarie, tra i quali, soltanto il capitello che

\footnotetext{
${ }^{2}$ Müller-Wiener 1977, 354, figg. 408-409.

${ }^{3}$ Al 1920 risale il ritrovamento del tutto casuale, durante lavori di canalizzazione, del fusto frammentario di una coloss ale colonna, alta oltre $4 \mathrm{~m}$, modellata in guisa di tronco arboreo nodoso, oggi sistemata nella corte del Museo Archeologico d'Istanbul (inv. 4568): Verzone 1956, Barsanti 1995, 14, fig. 6.

${ }^{4}$ Le indagini vennero condotte dalla Direzione dei Musei delle Antichità, congiuntamente alla missione archeologica inglese (Casson, Rice 1929, 36-40), all'interno del Simkeş Han dove fu possibile liberare solo una porzione dei due basamenti dell'arco sui quali gravavano le strutture dell'edificio centrale del complesso. I contemporanei lavori di ampliamento e di abbassamento del livello stradale di Ordu Caddesi rimisero in luce i frammenti della colonna coclide di Teodosio I reimpiegati nelle sostruzioni del cinquecentesco hamam del sultano Beyazit II, che si erge sull'opposto lato di Ordu Caddesi: cfr. Sande 1981; Firatli 1990, 27-29, cat. 55-61, tav. 22; Kiilerich 1993, 51-55, figg. 25-31; Yoncac1 Arslan 2015, 117-125, figg. 3.15, 3.17-18. L'edificio, recentissimamente restaurato, ospita il museo dedicato alla tradizione del 'bagno turco'. Nelle sale sono esposti anche vari frammenti marmorei di epoca bizantina, tra i quali anche il ben noto frammento della colonna di Teodosio rimosso dall'angolo sud-est dell'edificio, cfr. Bayezid II 2015.

${ }^{5}$ Nell'estate-autunno del 1957, l'ulteriore ampliamento di Ordu Caddesi decretò la demolizione di gran parte dell'ormai fatiscente Simkeş Han, consentendo di liberare interamente i basamenti dell'arco e di recuperare numerosi elementi architettonici, cfr. Duyuran 1958, 71-73; Lafontaine 1959-1960, 370-371, tav. II, fig. 3. Altre indagini vennero condotte nel 1967, in occasione della costruzione del nuovo edificio della biblioteca universitaria, sull'opposto lato nord di Ordu Caddesi, cfr. Naumann 1976, 117-132.

${ }^{6}$ In proposito cfr. Yaylalı Yildız 2008; Gül 2009, 152, 153, fig. 43.

${ }^{7}$ I grandi basamenti sono tra loro distanti $\mathrm{m} 7,26$.

${ }^{8}$ Un'ampia selezione di pezzi riportati alla luce nel 1928 è stata presa in esame da Verzone 1956.

${ }^{9}$ Perplessità al riguardo, in particolare sulla cronologia degli archi laterali, forse aggiunti nel corso del V sec., poi atterrati dal sisma del 558, sono state sinteticamente espresse da Müller-Wiener (1977, nella didascalia della fig. 298) e, più di recente, da Bardill (2004, 130).

${ }^{10}$ Barsanti 1995; Faedo 1998; Lampinen 2016; Barsanti in c.d.s..

${ }^{11}$ Il disagio deriva principalmente dal fatto che molti elementi architettonici sono assai spesso coinvolti, in modo anche pittoresco, nelle circostanti attività commerciali.

${ }^{12}$ In occasione di frequenti sopralluoghi ho avuto modo di individuare vari frammenti, almeno quattro, di altri capitelli, di cui solo uno è stato incluso nel catalogo di Zollt 1994, 113, cat. 291.
} 
chiamerò $\mathrm{A}^{13}$ (FIG. 2), può essere ragionevolmente attribuito alla fase originaria dell'arco, mentre gli altri tre, vale a dire i capitelli B, C e D, che già ad una prima osservazione risultano formalmente diversi, ${ }^{14}$ vennero quasi certamente messi in opera a seguito dei danni arrecati al monumento dal sisma del 447 o dal successivo incendio del $465 .^{15}$

Sotto tale prospettiva il capitello A costituisce una sorta di incunabolo dei capitelli corinzi costantinopolitani e, nel contempo, un importante referente cronologico ${ }^{16}$ per le produzioni seriali degli opifici del Prokonnesos, nel caso specifico, per quella dei capitelli di tipo II, secondo l'ormai storico ordinamento tipologico elaborato negli anni Trenta del secolo scorso da Rudolf Kautzsch. ${ }^{17}$

Stringenti affinità si rilevano - non a caso - in un esemplare trovato nel corso delle indagini condotte nelle antiche aree estrattive dell'isola di Prokonnesos (od. Marmara Adas1 $)^{18}$ (FIG. 3), le cui coordinate formali, come pure i dati dimensionali sono in tutto e per tutto sovrapponibili a quelli del capitello A. Elementi caratterizzanti la struttura e l'apparato ornamentale di entrambi i capitelli sono le due corone di otto foglie di acanto, a cinque lobi incavati e, soprattutto, le quattro foglie 'avvolgenti' che emergono, molto aderenti al kalathos, dalla corona superiore, da una specie di peduncolo triangolare, nel quale possiamo riconoscere il caulicolo atrofizzato. Le foglie 'avvolgenti' non sono altro che gl'involucri vegetali dei calici dispiegati a ventaglio sotto gli angoli dell'abaco, i cui lobi interni, alquanto sviluppati, s'incurvano quasi ad uncino verso il centro del kalathos, delimitando un rigonfiamento più o meno accentuato, a superficie liscia, sul quale, nel caso specifico del nostro capitello $\mathrm{A}$, è incisa una sigla di lavorazione composta da tre lettere greche ПА $\Lambda .{ }^{19}$

\footnotetext{
${ }^{13}$ Il capitello, notevolmente frammentario, venne trovato in occasione dello scavo del 1928: Verzone 1956, 131-132, fig. 4; Zollt 1994, 112-113, cat. 288, tav. 36; Kramer 1997, 42-43, fig. 21; Barsanti 2002, 1453, fig. 5; Russo 2007a, 8-9, fig. 26 (dove viene omesso il precedente contributo che trae spunto da una comunicazione presentata al convegno Ecclesiae urbis, sulla quale lo stesso Russo è tra l'altro intervenuto al termine della sessione: cfr. Guidobaldi, Guiglia Guidobaldi 2002, 1629-1631); Russo 2009a, 158, fig. 9.

${ }^{14}$ Differenze evidenziate anche da Zollt 1994, 113-114, cat. 289-292.

${ }^{15}$ Cfr. Müller-Wiener 1977, 262.

${ }^{16}$ La documentazione al riguardo è particolarmente esigua e molto problematica. Dubbia è in effetti la cronologia dei pochi isolati capitelli attributi al IV secolo, cfr. Betsch 1979, 186-187, 206, figg. 63, 130-131, 149; Zollt 1994, 111-112, 291-292, cat. 284-287, 583-585, tavv. 36 e 43; Russo 2007a, 10, fig. 43 (capitello pseudo composito, inv. 5072, nel giardino del Museo Archeologico, proveniente dall'area dell'Adalet Saray, prossima all'Ippodromo).

${ }^{17}$ Kautzsch 1936, 51-53; Betsch 1979, 55-56, 198-200; Kramer 1997, 19-28. Rudolf Kautzsch non poteva certo immaginare che, ancora oggi, a distanza di settant'anni, la sua classificazione avrebbe continuato ad essere un ineludibile punto di riferimento per lo studio dei capitelli corinzi costantinopolitani, pur se quel quadro cronologico sia stato sovente mod ificato da nuove scoperte. La prematura scomparsa di Annie Pralong lascia purtroppo incompiuta l'applicazione pratica del nuovo ordinamento tipologico dal lei elaborato per gli oltre 800 capitelli corinzi catalogati nella sua tesi di dottorato (Recherches sur les chapiteaux corinthiens tardifs en marbre de Proconnèse, Université de Paris I-Panthéon-Sorbonne 1997), che ha distribuito in quattro classi primarie, a loro volta suddivise in sottogruppi; cfr. in proposito Sodini 2000 , 427-428; Pralong 2000, 81-89; Barsanti 2002, 1450.

${ }_{18}$ Asgari 1995, 269 (nota 5), fig. 5; Barsanti 2002, 1452, fig. 4. Il capitello (Survey Oc 320, alto $134 \mathrm{~cm}$ ), dall'area di Silinte, si trova tra i materiali riuniti nella collezione della cava Ilik, dove ho potuto esaminarlo nel 2001.

${ }^{19}$ Diversi elementi dell'arco recano incise sigle di lavorazione, alcune già individuate da Casson e Rice (1929, 38, fig. 46), altre segnalate più recentemente da Bardill (2008, 209 e nota 50) e dalla Marsili (2014b, 184, fig. 6), la quale ha proposto d'interpretare la sigla TP, incisa, assieme alla sigla CI, sui blocchi marmorei di grandi dimensioni del basamento nord, come abbreviazione di TAUROU, alla stregua di marchio di destinazione riferito al toponimo utilizzato in antico per il foro teodosiano. Ipotesi interessante, ma poco convincente che deve tener anche conto delle altre attestazioni della stessa sigla, tra cui, quella incisa su un capitello corinzio di tipo VII proveniente dall'area della c.d. Casa di Giustiniano,
} 
Sui lobi esterni delle foglie 'avvolgenti', che si estendono a guisa di mensola, s'adagiano invece $\mathrm{i}$ brevi nastri delle volute schiacciate orizzontalmente sotto l'abaco, le quali si originano inorganicamente dalla foglia centrale della corona superiore. Per quanto riguarda l'articolazione delle foglie d'acanto, va altresì notato come i lobi inferiori delle fogliette mediane, piuttosto lunghe e lanceolate, si ripieghino, creando zone d'ombra a goccia allungata, mentre le altre, toccandosi, danno forma a figure geometriche sovrapposte, quadrangolari e pentagonali, che sembrano quasi prevaricarne i morfemi, prefigurando, in versione ancora embrionale, la cosiddetta 'maschera d'acanto', che, se pure già presente sugli esemplari microasiatici di II-III secolo, divenne una vera e propria cifra formale peculiare, connotante l'acanto dei capitelli corinzi costantinopolitani.

Il capitello del Prokonnesos offre anche lo spunto per alcune osservazioni sulle dinamiche organizzative e sulla prassi operativa del cantiere del foro teodosiano e, in particolare, sulle modalità di approvvigionamento dei materiali provenienti dalle cave dell'isola, i quali, assieme ai marmi di più lontana provenienza, venivano certo riuniti in un'area di stoccaggio, che dobbiamo immaginare situata in prossimità di uno scalo portuale, una statio marmorum come la Marmorata romana, ma della cui esistenza se pure ipotizzabile, non sono state ancora rintracciate evidenze archeologiche. ${ }^{20}$

Con tutta probabilità il cantiere veniva rifornito dagli opifici attivi nell'orbita delle cave del Prokonnesos sia di elementi prefabbricati, completamente rifiniti e pronti per l'impiego (quasi certamente i capitelli, come farebbe appunto pensare il citato esemplare del Prokonnesos), sia di materiali sommariamente sgrossati, come suggerirebbero varie cornici in uno stato di manifattura più o meno sommaria, ma opportunamente sagomate da completare $\mathrm{e}$ rifinire in situ, come appunto nel caso di alcuni segmenti della cornice di coronamento ${ }^{21}$ (FIG. 4). Significativa al riguardo può essere anche la testimonianza di un frammento di fusto di colonna, trovato anch' esso presso una cava del Prokonnesos, sul quale si distinguono appena abbozzati quei nodi che caratterizzano le colonne dell'arco, modellate a guisa di tronco arboreo. $^{22}$

Il corpus dei capitelli appartenenti alla categoria di tipo II, da ritenere senz'altro una tra le prime produzioni seriali delle botteghe del Prokonnesos in larga parte destinata alle erigende fabbriche della neocapitale, della quale ho già avuto occasione di proporre una prima analisi d'insieme, conta circa 70 esemplari, molti di grandi dimensioni, con altezze che oscillano tra $i$ $70 \mathrm{~cm}$ e il metro e oltre, come nel caso specifico dei capitelli dell'arco, i quali stando al nostro capitello A, raggiungevano eccezionalmente, come si è detto, i $135 \mathrm{~cm}$ di altezza.

oggi nel lapidario della Santa Sofia (a destra del vialetto d'accesso agli uffici del Museo): Barsanti 1989, 116, fig. 23; Karagiorgou 2015, 185, fig. 16.

${ }^{20}$ In proposito, cfr. Betsch 1979, 159-162. Nel corso del recente scavo dell'antico porto teodosiano nell'area di Yeni Kapı non sembra siano emerse evidenze di questo genere. È comunque lecito ipotizzare un diverso luogo di approdo sulle coste del Mar di Marmara, forse Heraclea Perinthos, sulla rotta verso la capitale.

${ }^{21}$ Oltre al frammento angolare pubblicato da Verzone (1956, 144, fig. 15), si segnala un altro frammento sulla cui sima è incisa la lettera A, identificabile come sigla di lavorazione, accanto al frammento angolare della fig. 11. Interessante è pure la testimonianza offerta dai due segmenti di architravi recuperati nel 1958 nell'area del Foro di Teodosio, sistemati all'esterno del Museo Archeologico, completamente rifiniti sul lato frontale e nel soffitto, mentre il lato posteriore presenta semplici sagomature predisposte, come la larga fascia convessa, per il fregio con i girali d'acanto: Barsanti 1982, 421-422, fig. 4; Milella 1996, 65, fig. 4; Russo 2007a, 4-5, figg. 14-16.

${ }^{22}$ Barsanti 1989, 101, fig. 8; Asgari 1989, 51, fig. 2 tav. 6,1; Barsanti, Guiglia, Paribeni 2010, 119, fig. 4. 
Circa una metà si concentra in area costantinopolitana, ${ }^{23}$ dove ricordo in particolare il gruppo di esemplari erratici nell'area di Saraçhane, ${ }^{24}$ per lo più considerati, e forse non a torto, spoglie delle vie porticate ricordate dalla Notitia urbis constantinopolitanae, ${ }^{25}$ ed inoltre, l'esemplare (FIG. 5) trovato nello scavo condotto nel 1935 da Alfons Maria Schneider nell'area dell'atrio giustinianeo della Santa Sofia, attribuito al portico del prospetto teodosiano della grande chiesa costantinopolitana, ${ }^{26}$ iniziato dopo il 404 e inaugurato, ricordo, il 10 ottobre del $415 .^{27}$ Degni di nota sono pure tre capitelli, anch' essi di notevoli dimensioni, nel Museo Archeologico di Kocaeli (Izmit-Nicomedia). ${ }^{28}$

Per quanto riguarda invece la diffusione della categoria, le attestazioni, non molto numerose, circa venticinque esemplari, si concentrano in area romana e nelle regioni del Mediterraneo sud orientale; mi sembra opportuno ricordare che molti capitelli corinzi di tipo II sono collegati a importanti progetti architettonici riconducibili direttamente o indirettamente ad interventi di munificenza imperiale, mi riferisco in particolare agli esemplari attribuiti alla fabbrica teodosiana della basilica romana di San Paolo f.1.m., ${ }^{29}$ e a quelli del grande santuario di San Mena nella Mareotide, nelle immediate vicinanze di Alessandria d'Egitto, la cui costruzione venne appunto realizzata con gli interventi finanziari di Arcadio e di Teodosio II. Parte di essi, in particolare i capitelli romani, parrebbero giunti sulle rive del Tevere in uno stato completo di lavorazione, laddove, in altri casi, è verosimile pensare che

\footnotetext{
${ }^{23}$ Cfr. Betsch 1979, 198; Zollt 1994, 112-118, cat. 288-302, fig. 8 e tavv. 36-37; Kramer 1997, 133-136; Barsanti 2002, 1455; Russo 2007a, 9, figg. 26-30, 33, 40. Assieme al piccolo gruppo in opera nella Yerebatan Saray1 (Barsanti 2002, 1455, fig. 7; Onlü 2010, 53-54, 110-112, figg. 4 e 14), meritano di essere ricordati, tra i meno noti: il capitello, di grandi dimensioni, fuori opera nell'area della Porta d'Oro (Betsch 1979, 198, fig. 144; Zollt 1994, 114, cat. 294, con datazione alla seconda metà del IV secolo), e il frammentato esemplare dallo scavo del peristilio del Grande Palazzo imperiale, oggi nel Museo dei Mosaici (Martiny 1947, 10-11, tavv. 9,1 e 13,1; Zollt 1994, 114, cat. 294; Kramer 1997, 135-136; Barsanti 2002, 1455).

${ }^{24}$ Cfr. Zollt 1994, 115-118, cat. 297-302; Barsanti 2002, 1454, fig. 6; Russo 2007a, 9, figg. 28-30. Complessivamente si contano circa cinquanta pezzi, tra capitelli corinzi di vario tipo, ionici ad imposta e semplici imposte, otto sono gli esemplari del tipo II.

${ }^{25}$ Betsch 1979, 172-182. Per le vie porticate si veda Berger 1997; Berger 2000; Yoncaci-Arslan 2015, $179-194$ (con bibliografia); Yoncaci-Arslan 2016.

${ }^{26}$ Schneider 1941, 8, n. 2, tav. 12; Deichmann 1956, 66; Betsch 1979, 194-195, fig. 138; Strube 1984, 84; Zollt 1994, cat. 293, 114, tav. 37; Kramer 1997, 41-42, 132 (cat. 1), fig. 20; Pralong 2000, 85, fig. 3; Barsanti 2002, 1452, fig. 5; Russo 2007a, 7, 9-10, fig. 22; Russo 2009a, 158, fig. 8; Russo 2010a, 25, fig. 11; Russo 2015, 304, fig. 6. Il capitello (inv. 134), alto $\mathrm{cm} \mathrm{82,} \mathrm{reca} \mathrm{incisa} \mathrm{sul} \mathrm{kalathos} \mathrm{la} \mathrm{lettera} \mathrm{A} \mathrm{capovolta,} \mathrm{evidentemente} \mathrm{una} \mathrm{sigla} \mathrm{di} \mathrm{lavorazione} \mathrm{apposta} \mathrm{nel}$ corso della sua manifattura, ed esattamente, stando allo schema delle 'workshop stages' elaborato da Asgari (1990, 115116, fig. 1), durante la fase III o V, quando il capitello era in posizione capovolta.

${ }^{27}$ Circa i tempi della ricostruzione della grande chiesa costantinopolitana dopo l'incendio del 20 giugno 404, cfr. le pertinenti riflessioni di Bardill 2004, 53-55. Per la storia e le evidenze monumentali dell'edificio prima della ricostruzione giustinianea vedi ora Taddei 2017.

${ }^{28}$ Oltre alla coppia di capitelli (inv. 2015-2016, alti rispettivamente 94 e $97 \mathrm{~cm}$ ), pubblicati dalla Pralong $1993,142 \mathrm{fig}$. 19, si veda il capitello da Sümer Ilk Okulu (alto cm 93) preso in esame da Kramer 1997, 138, n. 36, fig. 22.

${ }^{29}$ Kramer 1997, 3-28; Russo 2007a, 8 e nota 41, ritiene che i capitelli della basilica ostiense (Kramer 1997, 3-4, 68-72, 87-95, figg. 1-3) sarebbero stati realizzati a Roma da maestranze microasiatiche appositamente ingaggiate per l'edificio di fondazione imperiale. Non si esprime invece sulla manifattura degli altri esemplari romani che appaiono sostanzialmente identici. In proposito si tengano presenti le considerazioni di Brandenburg (2009, 185-189) che si esprime in favore dell'ipotesi di un'importazione di manufatti rifiniti, ipotesi che a suo avviso verrebbe peraltro confermata dai due capitelli trovati in prossimità del bacino esagonale di Porto e dal diciannovesimo esemplare recuperato nel 1995, sul fondo del Tevere, all'altezza dell'isola Tiberina, in prossimità della Marmorata, cfr. Pensabene 1995, 336-337, fig. 357; Brandenburg 2009, fig. 46.
} 
siano stati piuttosto commercializzati in uno stato di rifinitura parziale per essere completati e rifiniti con poche modifiche nei luoghi di destinazione da maestranze locali. Paradigmatica al riguardo è la testimonianza degli esemplari egiziani ${ }^{30}$ e gerosolimitani, ${ }^{31}$ di fattura assai omogenea, quasi certamente riconducibile ad una unica bottega attiva con ogni probabilità presso un centro di raccolta, di lavorazione e smistamento dei marmi importati, da localizzare in uno dei tanti scali portuali sulla costa mediterranea del Levante, tra i quali: Tiro, Cesarea, Gaza e soprattutto Alessandria, dove, fin dall'età imperiale, specie a partire dall'epoca severiana, confluivano i materiali marmorei provenienti dall'area egea, dal Prokonnesos o dall'Asia Minore. E infatti assai probabile che questi opifici, ai quali le maestranze giunte talora assieme ai carichi di marmo avevano trasmesso tecniche di lavorazione e repertori stilistici, fossero in larga misura riforniti di materiali semilavorati, senz'altro più economici e maggiormente remunerativi. ${ }^{32}$

Stando ai dati a nostra disposizione, la linea temporale della produzione dei capitelli di tipo II, avviata con ogni probabilità già nel corso del terzo quarto del IV secolo, non sembrerebbe protrarsi molto dopo il primo quarto del $\mathrm{V}$ secolo, ${ }^{33}$ quando avrebbe lasciato il posto ad una versione essenzializzata, ridotta nelle dimensioni e nell'apparato decorativo, configurata dagli esemplari del tipo cosiddetto 'a medaglione', la cui produzione perdurò invece a lungo, quasi certamente fino alla prima metà del VI secolo, documentata da un gran numero di attestazioni disseminate in quasi tutte le regioni del Mediterraneo e del Mar Nero. La larga diffusione dei capitelli corinzi 'a medaglione', per i quali ho tra l'altro manifestato in più occasioni un particolare interesse, può essere del resto giustificata anche, e soprattutto, da una struttura decorativa viepiù semplificata, che ne poteva certo abbreviare i tempi di lavorazione nel quadro di una produzione massificata, organizzata su scala industriale, come lo era appunto quella degli opifici del Prokonnesos. ${ }^{34}$

Tornando all'arco del foro di Teodosio, vorrei, se pur rapidamente, riesaminare gli altri tre capitelli, B, C e D, quasi certamente messi in opera, come si è detto, in occasione di un seriore restauro del monumento, realizzato nel corso della seconda metà del V secolo.

La precedenza spetta all'esemplare B, immortalato nella 'pittoresca' immagine pubblicata nel breve rendiconto delle indagini del $1928^{35}$ e in seguito nella bella foto del novembre 1945

\footnotetext{
${ }^{30}$ Cfr. Kautzsch 1936, nn. 81, 82, 83, 88, 96, tav. 6. Si veda anche un capitello nel giardino delle catacombe di Kom el Chougafa (inv. 144) ad Alessandria: Pralong 2000, 89, fig. 12.

${ }^{31}$ Mi riferisco in particolare a due capitelli che si trovano sulla spianata dell'Haram al-Sharif, in prossimità del Museo Islamico: Wilkinson 1987, 106-109, cat. 60-63; Kramer 1997, 136-137, n. 38.

${ }^{32}$ Sodini 2000, 427. Sull'importazione dei materiali marmorei nelle regioni del Mediterraneo orientale cfr. Fis cher 1998; Kahwagi-Janho 2014. Più in generale sul fenomeno della diffusione dei manufatti marmorei, che vede confrontarsi ipotesi diverse sulla mobilità dei materiali o delle maestranze, cfr. Russo 2010b. L'ipotesi, avanzata a suo tempo da Pensabene $(1986,348)$ circa il presunto stato di parziale lavorazione dei 28 capitelli corinzi trasportati dalla nave naufragata a Marzamemi, è stata accolta da Russo (2006a, 245; 2010b, 37-38; vedi anche Leidwanger 2018, 344). A suo avviso quei capitelli potevano essere messi in opera 'non finiti', anche se è piuttosto verosimile pensare ad un completamento nei luoghi di destinazione, come verrebbe provato da due capitelli della chiesa ovest di Apollonia in Cirenaica che mostrano la parte superiore del kalathos maldestramente rifinita da artefici locali. Ma l'esempio suggerito è ambiguo e inadeguato, poiché i capitelli indicati sono del tipo 'a lira', tra l'altro abbinati nello stesso edificio a capitelli analoghi a quelli di Marzamemi, i quali non mostrano segni di rifinitura locale, come, del resto, quasi tutti gli esemplari appartenenti a questa categoria, quella di tipo VII, per la quale cfr. Barsanti 1989, 111-123.

${ }^{33}$ Betsch 1979, 56.

${ }^{34}$ In proposito Barsanti 2014 e Barsanti in c.d.s..

${ }^{35}$ Casson, Rice 1929, 38, fig. 45; Barsanti 1995, 14, fig. 9.
} 
di Nicholas Artamonoff ${ }^{36}$ (FIG. 6). Del monumentale capitello restava grosso modo una metà, ${ }^{37}$ che allo stato attuale si è ancor più ridotta: ne sopravvive infatti solo una porzione riconoscibile nel sostegno sinistro dei due segmenti di cornici sistemati lungo Ordu Caddesi (FIGG. 7-8a-b). L'altro sostegno è invece rappresentato dal capitello C, anch'esso in condizioni frammentarie, il quale, come mostra una ripresa fotografica del 1970 dell'archivio Betsch, ${ }^{38}$ ha preservato solo la corona superiore di foglie di acanto ${ }^{39}$ (FIG. 9a-b). L'esemplare D (FIG. 10), collocato sul marciapiede di Ordu Caddesi, introduce all'area archeologica dell'arco chi da Beyazıt Meidanı si dirige verso Aksaray. Anch'esso in condizioni frammentarie, presenta una superficie scolpita notevolmente degradata. ${ }^{40}$

Le differenze più marcate rilevabili nelle coordinate formali di questi tre esemplari rispetto al capitello A sono le volute a spigolo vivo, ben evidenti nel capitello B, esito della convergenza di due piani spioventi, per lo più caratterizzanti la struttura degli esemplari corinzi di manifattura seriore. È da notare inoltre l'accentuata schematicità della maschera d'acanto, che attenua in modo sensibile quel naturalismo ancora apprezzabile nell'acanto nel capitello A, la cui specifica morfologia, formalmente ben delineata, può essere a mio avviso avvicinata a quella che si distingue, malgrado i guasti e le cerchiature di metallo, sul kalathos del grande capitello della colonna dedicata dal praefectus urbi Taziano (450-c.452) all'imperatore Marciano (450-457), ${ }^{41}$ siamo dunque nel sesto decennio del V secolo. La stessa formula diagrammatica caratterizza pure l'acanto del colossale capitello, alto 2,38 m (FIG. 11), attribuito convincentemente da Urs Peschlow alla colonna eretta in onore del successore di Marciano, l'imperatore Leone I (457-474), ${ }^{42}$ nel suo foro ai Pittakia. ${ }^{43}$

Tra i materiali dell'arco disordinatamente ammassati nelle aiuole di Ordu Caddesi e nel nuovo 'spazio' archeologico, allestito di recente (2016), poco oltre il Simkeş Han, vi sono diversi frammenti dei soffitti dell'intradosso dell'arco, una categoria che allo stato attuale non sembrerebbe altrimenti documentata in area costantinopolitana, caratterizzati da cassettoni rettangolari a semplici gradini, sia del tutto lisci, ${ }^{44}$ sia con inserti vegetali e animali ${ }^{45}$ (FIG. $12 a-b)$.

Senza soffermarmi su altri elementi architettonici dell'arco, vorrei piuttosto concentrare l'attenzione sulla cornice di coronamento, del classico tipo a mensole, convenzionalmente

\footnotetext{
${ }^{36}$ Cfr. Dumbarton Oaks N. Collection: CFA.NA 0341.

${ }^{37}$ Zollt 1994, 113, cat. 289, tav. 37.

${ }^{38}$ Cfr. William Earl Betsch Photographs of Architectural Capitals in Istanbul, 1970, PH,.BZ.002, Image Collection and Fieldwork Archives, Dumbarton Oaks, Trustees for Harvard University, Washington, D.C.

${ }^{39}$ Zollt 1994, 113, cat. 290 (alt. cm 130). Sui capiteli pertinenti all'arco di Teodosio vedi pure Taddei 2017, 177-178, figg. 74-76.

${ }^{40}$ Zollt 1994, 114, cat. 292 (alt. 175 ?), tav. 37.

${ }^{41}$ Il capitello è alto cm 149: Kautzsch 1936, 47-49, figg. 3-4, tav. 11; Kollwitz 1941, 69-70, 74-75 (Exkurs di P. Schazmann), tav. 10/11; Peschlow 1986, 26-28, tav. 5; Zollt 1994, 203, cat. 586, tav. 43.

${ }^{42}$ Peschlow 1986; Zollt 1994, 203, cat. 587, tav. 44; Russo 2008, 69-70, figg. 46-48. Sulla colonna, caratterizzata da un fusto composito, era con tutta probabilità collocata la colossale statua bronzea di Barletta nella quale Peschlow ha infatti proposto di riconoscere l'imperatore Leone I. Circa le molte identificazioni proposte per l'enigmatico personaggio ritratto nella statua, cfr. le riflessioni di Kiilerich 2015.

${ }^{43}$ Per l'ubicazione dei Pittakia a Sud-Est della Santa Sofia cfr. Dark, Harris 2008.

${ }^{44}$ Verzone 1956, 154-155, figg. 26-27.

${ }^{45}$ Duyuran 1958, fig. 3 (in secondo piano).
} 
definita anche cornice 'a modiglioni', che s'impostava su due cornici, ${ }^{46}$ 1'una decorata con un fregio di elementi baccellati e una paratattica sequenza di foglie d'acanto del così detto tipo molle, caratterizzate da una fattura piuttosto frettolosa, quasi sciatta, ${ }^{47}$ mentre l'altra mostra semplici fasce lisce sovrapposte in leggero risalto prospettico ${ }^{48}$ (cfr. FIG. 7). Di entrambe sono stati ritrovati vari frammenti, anche, come si è detto, non finiti. ${ }^{49}$

Il pezzo più completo della cornice di coronamento è un frammento angolare (FIG. 13), il quale presenta una sintassi decorativa canonica, ma nettamente semplificata, ridotta all'essenziale: restano solo le foglie d'acanto scolpite sulle mensole di forma squadrata, poste su un liscio risalto sagomato, predisposto evidentemente per il kyma ionico, e ornati di carattere vegetale nei piccoli lacunari. ${ }^{50}$ Il repertorio degli inserti doveva essere piuttosto vario, comprendente fiori, pomi e melograni, nonché figure zoomorfe, come mostra appunto un frammento decorato con una testa di capro (FIG. 14).

A differenza del modello tradizionale caratterizzante l'ordine corinzio, documentato da più di un esempio di epoca imperiale, ${ }^{51}$ con mensole, quasi sempre contraddistinte da una sagoma ad esse, tutte ortogonali, anche in corrispondenza degli angoli, il frammento dell'arco esibisce una mensola di forma pentagonale sulla diagonale dell'angolo.

È stato il Detweiler ad attirare per primo l'attenzione su questa variante, rilevata nelle cornici di coronamento del monumentale arco di Adriano a Gerasa, ${ }^{52} \mathrm{e}$ in seguito presa in esame dal Ginouvés in relazione alle cornici del ninfeo di Laodicea sul Lykos, datato al tempo di Caracalla (212-223). ${ }^{53}$ Entrambi ne hanno tracciato per somme linee il quadro di diffusione, inquadrandone l'origine nelle regioni dell'Oriente mediterraneo, dove molteplici sono in effetti le attestazioni, a partire dall'età adrianea. ${ }^{54} \mathrm{Ne}$ è stata parallelamente sottolineata l'ampia presenza in Asia Minore, testimoniata da un gran numero di esempi concentrati prevalentemente nelle regioni del Sud-Est, soprattutto la Cilicia e la Panfilia, ma anche altrove, in Pisidia, in Frigia e in Galazia. ${ }^{55}$

È del resto assai probabile che il successo delle cornici di coronamento con la mensola diagonale, che ben si coniugano con l'articolato vocabolario architettonico degli edifici tardo antonini e severiani, sia in qualche modo collegato agli spostamenti di équipes di eterogenee maestranze itineranti, attratte dai cantieri di prestigiosi edifici pubblici in corso d'opera, tra $i$ quali, per citare le imprese più importanti e rappresentative, gli scenografici ninfei di Perge e

\footnotetext{
${ }^{46}$ Come mostra appunto il disegno del Verzone 1956, 146, figg. 18 e 19.

${ }^{47}$ Verzone 1956, 142-144, figg. 12 e 13; Barsanti 1995, 20, fig. 23; Russo 2007a, 4, fig. 11.

${ }^{48}$ Verzone 1956, 147-151, figg. 20-24.

${ }^{49} \mathrm{Si}$ veda anche un altro frammento angolare pubblicato da Verzone 1956, 144, fig. 15.

${ }^{50}$ Verzone 1956, 144-146, figg. 14-17, 19; Barsanti 1995, 19, fig. 22; Russo 2007a, fig. 12.

${ }^{51}$ Tra i molti esempi basterà citare l'arco augusteo di Rimini e il tempio romano di Vespasiano.

${ }^{52}$ Detweiler 1938, 78, tav. XIa.

${ }^{53}$ Ginouvès 1969, 113, 120ss, nn. 1251, 1743b, 1790bis, tavv. 59,4, 60,1; Milella 1996, 72, fig. 13; Şimşek 2007, 342 , figg. 138, 140 .

${ }^{54}$ Tra le molte testimonianze relative alla diffusione della variante in questione in area mediorientale, ricordo le cornici del tempio di Venere a Baalbek, del teatro di Daphne nelle vicinanze di Antiochia, della Kalibé di Bosra, del tetrapilo e del tempio di Balshamin a Palmira, nonché le cornici di Tartous e di Cesarea di Palestina. Parrebbe invece assente in Egitto dove si può tuttavia segnalare l'isolato esempio di una cornice a modiglioni di II secolo trovata nel 'piccolo teatro' di Kom el-Dikka ad Alessandria, che reca sulla diagonale dell'angolo il busto di una figura femminile: McKenzie 2010, 210, fig. 362.

${ }^{55}$ Per un'ampia documentazione al riguardo, cfr. Türkmen 2007.
} 
di Sagalassos, il teatro di Hierapolis e il tempio di Zeus ad Aizanoi, senza però raggiungere le grandi città della Asia Minore occidentale, dove, al momento, tale variante non parrebbe attestata. Ho comunque potuto accertarne la presenza in Bitinia, ed esattamente, a Nicomedia, testimoniata da un frammento angolare di cornice di età adrianea nel Museo Archeologico di Kocaeli (Izmit/Nicomedia) (FIG. 15). Ciò non sorprende poiché, raccogliendo l'ipotesi a suo tempo formulata dal Ward Perkins, nella città doveva operare un grande centro, organizzato su scala industriale, di raccolta, lavorazione e distribuzione dei manufatti marmorei, un vero e proprio crogiolo di esperienze e provenienze diverse. ${ }^{56} \mathrm{Ed}$ è verosimile pensare che dalla Bitinia la mensola diagonale sia sbarcata a Bisanzio prima e a Costantinopoli poi, dove tale modello sintattico venne adottato sul volgere del IV secolo nell'arco di Teodosio, e, a distanza di pochi anni, nella elegante cornice di coronamento del fastigio del prospetto teodosiano della Santa Sofia (FIG. 16a-b), realizzato, come si è detto, tra il 404 e il 415.

Eccettuata la breve menzione che ne fa Ginouvés, rimarcando la presenza di questa variante nel decoro architettonico di Costantinopoli, esemplificata appunto dalle cornici della Santa Sofia, l'argomento non sembra aver destato altrimenti interesse. Eppure, la variante in questione non può non attirare l'attenzione, specie nel caso del frammento dell'arco, in bella vista tra i materiali di Ordu Caddesi e, soprattutto, nei due grandi elementi angolari della cornice di coronamento del fastigio della Santa Sofia teodosiana, dai cui vertici si protende una mensola diagonale. ${ }^{57}$

A differenza della sobria ed essenziale sintassi decorativa che caratterizza il frammento dell'arco, le cornici della Santa Sofia teodosiana, riportate alla luce dallo Schneider, ripropongono con una certa fedeltà e con grande ridondanza tutto l'apparato decorativo 'canonico' delle cornici dell'ordine corinzio di tradizione classica: dall'elegante fregio con foglie lanceolate alternate a dardi sulla sima ai dentelli, dagli astragali al kyma ionico ${ }^{58}$ che ne incornicia lacunari e mensole, molte con sagomatura 'a esse' appena accennata e rocchetto terminale provvisto di balteo, mentre in altre, di forma più squadrata, la sagomatura viene semplicemente simulata da una 'esse' riprodotta a basso rilievo su entrambi i lati della mensola. I piccoli lacunari accolgono una vasta gamma di ornati, prevalentemente elementi vegetali, ma anche suppellettili, come anfore, brocche e vasi, nonché una croce e almeno in un caso, un'aquila. ${ }^{59} \mathrm{Si}$ avverte tuttavia una perdita di coerenza organica nella metrica compositiva e nei significati strutturali dei rapporti proporzionali dei piani prospettici, variazioni per le quali si rinvia alle pertinenti osservazioni e ai confronti a suo tempo suggeriti da Deichmann, sia per il nobile disegno architettonico del prospetto, sia per le singole membrature e i relativi decori, in larga misura debitori delle grandi tradizioni

\footnotetext{
${ }^{56}$ Ward Perkins 1980. È del resto assai probabile che provenissero proprio da Nicomedia molte maestranze impegnate, sul volgere del III secolo, nel cantiere del palazzo di Diocleziano a Spalato, dove ho di recente potuto constatare l'estesa presenza di cornici con mensole diagonali nelle trabeazioni del colonnato del peristilio, negli architravi dei portali del mausoleo, dell'atrio monumentale del palazzo e del tempio di Zeus.

${ }^{57}$ Schneider 1941, 13, n. 11a-b, fig. 5, tav. 21; Deichmann 1956, 66-67, figg. 12 e 14; Butler 1990, 84-87, figg. 28-32; Russo 2007a, 12, fig. 54; Russo 2010a, 27, figg. 17-18; Russo 2015, figg. 11-12; Taddei 2017, 186, figg. 99-100.

${ }^{58}$ Schneider 1941,15 , n. 11c-e, tav. 22. In corrispondenza degli angoli interni, il fregio è talora arricchito da inserti vegetali.

${ }^{59}$ Un'aquila decora il soffitto di un frammento di cornice curvilinea (inv. 23): Schneider 1941, 15, n. 11e, tav. $22,3$.
} 
dell'Asia Minore, il quale non ha mancato di evidenziarne nel contempo le novità. ${ }^{60}$ Ed in effetti i legami con l'Asia Minore sono molteplici e densi d'implicazioni, tali da meritare un'opportuna revisione e, soprattutto, un meditato aggiornamento alla luce di molte nuove scoperte intervenute d'allora. ${ }^{61}$

Nel riprendere in esame i materiali del prospetto teodosiano, emersi dallo scavo dello Schneider, è opportuno ricordarne anche il carattere composito e in qualche modo eterogeneo, un aspetto questo che potrebbe anche lasciare spazio all'ipotesi di riconoscere in alcuni di essi le spoglie della precedente fabbrica inaugurata nel 360, al tempo di Costanzo II $(337-361),{ }^{62}$ alla quale Patrizio Pensabene ha appunto a suo tempo attribuito ${ }^{63}$ il già ricordato capitello corinzio di tipo II. È un'ipotesi seducente, suggerita soprattutto dalle coordinate formali del capitello che in effetti non trovano riscontro negli stilemi che caratterizzano i capitelli del fastigio teodosiano, e che dunque sotto tale prospettiva potrebbero anche implicare una distanza cronologica. Pur tuttavia va tenuto conto che anche nella prassi organizzativa del cantiere teodosiano della Santa Sofia era quasi certamente previsto un approvvigionamento di materiali 'prefabbricati', lavorati in serie, come appunto il capitello in questione ed anche gli altri tre capitelli corinzi, ${ }^{64}$ di tipo diverso, ma ugualmente di manifattura seriale, ${ }^{65}$ i quali possono essere considerati come resti di quantitativi più consistenti, gli uni destinati alle colonne del portico, gli altri, forse, delle navate dell'edificio ecclesiale. ${ }^{66} \mathrm{Nel}$ contempo, erano certo preventivati rifornimenti di materiali sommariamente lavorati, ma opportunamente sagomati, che sarebbero stati completati in situ, come lascia ad esempio supporre la cornice del fastigio (FIG. 17), non finita sul retro, con lisci risalti gradinati, e sul lato breve che presenta lisce sagomature predisposte per i fregi; da notare, su

\footnotetext{
${ }^{60}$ Deichmann 1956, 63-69, figg. 9-16, ed inoltre Grabar 1963, 44-58, tav. 18; Butler 1990, 84-87, figg. 29-32; Milella 1996, 61-65, figg. 1-3; Russo 2007a, 12-13, figg. 54-58; Russo 2015, 305-306, figg. 11-12. Vedi anche Niewöhner 2017, 257, fig. 34.

${ }^{61}$ Schneider 1941, 13, fig. 5, tav. 21, 1-2.

${ }^{62}$ Circa le testimonianze testuali relative alla fase 'costantiniana' della Santa Sofia. cfr. Westall 2011.

${ }^{63}$ Pensabene 1993, 407.

${ }^{64}$ Schneider 1941, nn. 4-5, 18, tavv. 23,4 e 25, 1-3; Pralong 1993, 137, fig. 4; Zollt 1994, 122, cat. 313; Kramer 1997, 137-138, figg. 26 a-b: Barsanti 2002, 1462, fig. 11; Russo 2007a, 7-8, figg. 23, 24-25; Russo 2009b, 25-26, figg. 26-27.

${ }^{65}$ I tre capitelli rientrano nel tipo I, grosso modo contemporaneo al tipo II (Kautzsch 1936, 51-52, 65-67; Betsch 1979, 200-202; Kramer 1997, 28-30). Presentano una struttura essenzializzata, di fattura alquanto 'frettolosa', con due corone di foglie di acanto 'molle', dalle quali fuoriescono i nastri delle volute. La loro produzione si prolunga fino, o poco oltre, la metà del V secolo, come indicherebbe la serie di esemplari già in opera nella distrutta cisterna presso la basilica costantinopolitana di San Giovanni di Studio, edificata intorno alla metà del secolo (Betsch 1979, 196-197; Kramer 1997, 139-140, figg. 27-34, 36). Allo stato attuale si contano almeno settanta capitelli di tipo I, di cui una metà in area costantinopolitana, molti riutilizzati nelle cisterne sotterranee (Barsanti 2013a, 1490-1491, fig. 6); numerosi sono anche gli esemplari erratici nell'area di Saraçhane (Zollt 1994, cat. 303-312, 313-324, 118-124, fig. 9, tav. 38; Kramer 1997, 118-124, fig. 9, tav. 38; Barsanti 2002, 1463, fig. 12; Russo 2007a, 9, figg. 29, 31-33, 39), tra i quali anche due capitelli doppi per colonne abbinate che si trovano lungo il lato ovest di Atatürk Bulvarı: l'uno è sistemato sopra un grande elemento di trabeazione marmorea ormai del tutto interrata (cfr, infra), come pure l'altro capitello di cui affiora dal terreno solo la base (Zollt 1994, 48-49, cat. 104-105; Russo 2007a, 9, fig. 35). Un valido raffronto è fornito da un esemplare, ugualmente di tipo doppio, trovato nell'isola di Prokonnesos (Survey Oc.250), ora nella collezione della cava Oğuz (Asgari 1995, 269 e nota 9, fig. 6).

${ }^{66}$ Betsch (1979, 10 e 199) ritiene che i capitelli, come del resto tutti gli elementi architettonici, vennero ordinati agli opifici del Prokonnesos non appena prese avvio il progetto di ricostruzione dell'edificio «to not delay construction, therefore the dating of ca. 405».
} 
questo stesso lato, l'incasso funzionale al suo montaggio nel lato sinistro del fastigio ${ }^{67}$ (FIG. $18)$.

Dobbiamo pertanto immaginare di vedere all'opera una o più équipes di maestranze, tra cui si distingue il gruppo di artefici responsabili della progettazione e della realizzazione del monumentale prospetto architettonico porticato, i quali, connotati da una competenza tecnica di alto livello, seppero rinnovare con equilibrio compositivo e grande eleganza formale la sintassi decorativa di tradizione classica. ${ }^{68}$ Stilemi che caratterizzano appunto, come si è detto, la struttura e l'apparato decorativo delle cornici con mensole, ma anche e soprattutto il fregio con girali di acanto sulle cornici del fastigio ${ }^{69}$ (cfr. FIG. 17), dove, a differenza delle composizioni di età classica, i girali non si svolgono più liberamente, con apparente facile naturalezza e con sfumati effetti chiaroscurali; essi si compongono e si avvolgono ritmicamente, in modo ordinato, ritagliati su una superficie convessa con una resa a risentito sottosquadro che simula e prelude una più elaborata lavorazione a giorno. ${ }^{70}$

Non dissimili appaiono i caratteri formali del superbo capitello corinzio (FIG. 19), l'unico superstite dei quattro del fastigio, ${ }^{71}$ attualmente collocato su un rocchio di colonna di marmo cipollino $^{72}$ al centro del lapidario dell'Ayasofya Müzesi dove sono riuniti gli elementi architettonici del prospetto teodosiano provenienti dallo scavo Schneider, tra i quali risaltano i frammenti degli stipiti e dell'architrave del portale centrale, per il quale venne utilizzata la puddinga di Hereke, caratterizzata da una policromia giallo-rosata, ${ }^{73}$ i monumentali lacunari con cassettoni di forma ottagonale ${ }^{74}$ e le cornici del portico, l'una decorata con una fitta sequenza di elementi baccellati, ${ }^{75}$ mentre sul piano di fondo leggermente concavo della sima dell'altra cornice è ritagliata con esiguo risalto una elegante serie di palmette, il classico anthemion, alternatamente aperte e chiuse, ognuna inscritta entro una incorniciatura in forma di scudo. ${ }^{76}$ A proposito di questi materiali, mi sembra opportuno osservare che, se si eccettua la già menzionata sigla di lavorazione incisa sul capitello corinzio attribuito al portico, nessun alto elemento del prospetto teodosiano presenta sigle di assemblaggio o di lavorazione.

Le proporzioni perfettamente calibrate delle due corone con otto foglie d'acanto, che avvolgono il kalathos del grande capitello del fastigio, si articolano con grande naturalezza,

\footnotetext{
${ }^{67}$ Schneider 1941, 11, fig. 4. È stata anche opportunamente contenuta in lunghezza la lavorazione della sagomatura nel soffitto (Ibidem, tav. 18,2). Quasi tutti gli elementi del fastigio sono provvisti d'incassi funzionali al loro assemblaggio e messa in opera. Sul pezzo vedi pure Niewöhner 2017, 257, fig. 35.

${ }^{68}$ Russo (2009b, 25, figg. 23-24), ravvisa un significativo precedente per la produzione di questa officina in un 'singolare capitello corinzio', più propriamente definibile 'pseudo composito', di IV secolo nel Museo Archeologico di Istanbul (inv. 5072: Zollt 1994, 202, cat. 585, tav. 43; Kramer 1997, 44, tav. 10,1).

${ }^{69}$ Schneider 1941, 11, n-7, tavv. 17,2, 18,2, 19 (gli elementi curvilinei).

${ }^{70}$ Russo 2010a, 27, figg. 14-15; Russo 2015, 305 fig. 9.

${ }^{71}$ Il capitello è alto 105 cm: Schneider 1941, 10, n. 5, tav. 14,2; Zollt 1994, 138, cat. 372, tav. 39; Russo 2007 a, 8, fig. 23-25; Russo 2009b, 25, fig. 25; Russo 2010a, 24, fig. 9; Russo 2015, 303, fig. 4.

${ }^{72}$ Erano appunto di marmo cipollino le colonne in opera nel portico e nel fastigio. Di esse resta anche un altro frammento, di maggiori dimensioni, dal quale è stata tuttavia resecata un'ampia porzione. Sulla policromia dei materiali delle membrature architettoniche del prospetto teodosiano, si sofferma brevemente Russo 2015, 107-108.

${ }^{73}$ Sulla storia dell'impiego a Costantinopoli della puddinga proveniente da Hereke, località nelle vicinanze di Izmit/Nicomedia, cfr. Ducrot 1998.

${ }^{74}$ Schneider 1941, 13, tav. 20; Russo 2010a, 30, fig. 19; Russo 2015, 306, fig. 13.

${ }^{75}$ Schneider 1941, 8-9, tav. 13,1-3; Deichmann 1956, 57-58, fig. 15; Russo 2010a, 26, fig. 12; Russo 2015, 304-305, fig. 7.

${ }^{76}$ Schneider 1941, 9, tav. 13, 4; Deichmann 1956, 58, fig. 15; Barsanti 1982, 421; Russo 2010a, 27, fig. 13; Russo 2015, 305 , fig. 8 .
} 
espandendo i lobi sommitali leggermente ondulati, che proiettano la loro ombra sulle sottostanti 'maschere d'acanto', dove il naturalismo e l'organicità dei morfemi vegetali si dissolvono in una formula diagrammatica, di valenza prevalentemente decorativa. Ogni foglia è percorsa da profonde solcature che s'incurvano e si congiungono, delineando con ritmica esattezza le sagome della maschera d'acanto, all'interno della quale i lobi delle fogliette, ripiegandosi, generano occhielli circolari, mentre, appena toccandosi, formano figure geometriche, rettangolari e romboidali. Dai calici, provvisti di cauli atrofizzati, nascono le volute e le elici, i cui sottili steli, uniti da una fascetta, s'incurvano sotto il fiore d'abaco, e si avvolgono conclusi da una foglia triloba, un arricchimento di sapore squisitamente classico. I nastri delle volute, desinenti in spirali, erano completamente distaccati dal kalathos, di essi, data la fragilità, ne resta almeno in parte solo uno. ${ }^{77}$

L'esame ravvicinato del capitello consente di apprezzare alcuni dettagli esecutivi, come, ad esempio, la lieve solcatura semicircolare intorno al bordo profilato degli occhielli, ${ }^{78}$ presente anche sugli altri elementi architettonici del prospetto teodosiano, che a mio parere può essere considerata alla stregua di una vera e propria 'cifra' di bottega.

Dei quattro capitelli di pilastro del prospetto ne restano solo tre, di cui due pressoché integri: l'uno (inv. 19) sistemato in prossimità dei due segmenti della cornice curvilinea del fastigio (FIG. 20), l'altro (inv. 83) si trova invece nella fossa del cantiere dello scavo 'musealizzato' dello Schneider (FIG. 21) di fronte alla facciata occidentale della chiesa giustinianea, dove, presso l'ingresso centrale, è sistemato il terzo capitello (FIG. 22) del quale resta tuttavia solo una porzione mutila della parte superiore. ${ }^{79}$

I capitelli hanno due corone di otto foglie d'acanto, quattro sulla fronte e due su ciascun lato, paratatticamente allineate su due registri, i cui morfemi vegetali parrebbero resi in modo più schematico rispetto a quelli del capitello, accentuando il valore ornamentale della composizione, affidata appunto alla configurazione simmetrica delle foglie intimamente fuse insieme nelle 'maschere d'acanto'. Va comunque tenuto conto che tale effetto viene in qualche modo accentuato dalla scomparsa dei lobi sommitali delle foglie di acanto quasi tutti fratturati e, soprattutto, dalla aderenza e appiattimento delle foglie disposte su uno stesso piano lineare, laddove nel capitello l'effetto veniva in qualche modo attenuato dalla superficie curva del kalathos.

Una serie di cinque elementi baccellati nel capitello inv. 83, ovaliformi, concavi e profilati nel capitello inv. 19, in entrambi i casi intercalati da sottili dardi rivolti verso l'alto, occupano, sopra la corona superiore, lo spazio centrale della fronte, lasciato libero dai calici spostati lateralmente. ${ }^{80}$

\footnotetext{
${ }^{77}$ Almeno fino al 2008 il fragile nastro della voluta superstite era intatto, come mostra appunto la tav. 39 di Zollt 1994.

${ }^{78} \mathrm{Nel}$ quale potremmo forse riconoscere il cosiddetto 'ringed void', un dettaglio che caratterizza sovente l'acanto dei capitelli classici ma anche quello di numerosissimi esemplari di V-VI secolo, soprattutto di ambito ellenico; per i quali, cfr. Déroche 1987.

${ }^{79}$ Schneider 1941, 10-11, n. 6, tavv. 15-16, 23,5: Deichmann 1956, 63-64, figg. 7-8; Russo 2007a, 5, fig. 18; Russo 2009a; Russo 2010a, 24, fig. 10; Russo 2015, 303-304, fig. 5.

${ }^{80}$ Ad avviso di Russo $(2010 \mathrm{a}, 26)$ «i capitelli di pilastro di S. Sofia diventerebbero 'compositi' grazie all'inserzione d'una sorta di kymation con ovoli bordati in negativo tra lancette dalla punta in alto (inv. 19) o da un susseguirsi di baccellature pure intervallate da lancette con la punta in alto (inv. 83)». Va tuttavia os servato che gli inserti rendono, per così dire, 'composito' solo l'apparato decorativo del capitello. Lo stesso fraintendimento ricorre anche nel catalogo delle sculture della prima basilica di San Giovanni a Efeso, dove i capitelli corinzi vengono sorprendentemente definiti compositi da Russo in ragione degli ornati che trovano posto tra le volute (Russo, Büyükkolanci 2010).
} 
Facendo scorrere lo sguardo sulla superficie scolpita del capitello inv. 19 risaltano alcuni dettagli della lavorazione che nel caso specifico non è stata rifinita. Quasi tutta la metà superiore del capitello mostra infatti una superficie scabra (FIG. 23), con estesi segni di scalpello a pettine; vi sono pure tracce di 'non finito', sia sul lato frontale, in particolare sotto l'abaco, dove non è stato del tutto eliminato il materiale superfluo, sia sul lato sinistro, dove vi sono i calici ma non i nastri delle elici e delle volute, entrambi presenti invece sul lato opposto; le elici terminano, come quelle del grande capitello corinzio, con una foglia triloba; le spirali delle volute non sono lavorate a giorno. Diversamente la superficie dell'altro capitello e anche quella del frammento parrebbero perfettamente rifinite.

Anche negli occhielli dell'acanto dei capitelli in questione si nota una lieve solcatura semicircolare intorno al bordo profilato, particolare che caratterizza pure l'acanto dei quattro capitelli di lesena in opera nella Porta d'Oro i cui stilemi sono stati a ragione avvicinati a quelli dei capitelli di pilastro della Santa Sofia. ${ }^{81}$ Resta invece praticamente isolato nel panorama costantinopolitano il grande capitello corinzio, che non sembra in effetti trovare adeguati termini di raffronto, eccettuato forse un esemplare di grandi dimensioni (FIG. 24), recentissimamente scoperto assieme ad altri architettonici nel cantiere di un supermercato a Gaza, nel quale possiamo senza esitazioni riconoscere un manufatto proveniente dalle botteghe del Prokonnesos. ${ }^{82}$ Lo scavo ha riportato alla luce anche i resti di un edificio, forse la celebrata cattedrale della città, costruita proprio nei primi anni del V secolo con munifiche sovvenzioni imperiali. E l'identificazione verrebbe in qualche modo corroborata dal ritrovamento di vari fusti di colonne di marmo verosimilmente caristio (cipollino), nei quali sarebbe in verità seducente riconoscere proprio quel gruppo di trentadue colonne che, stando alla testimonianza delle fonti venne appunto inviato in dono dall'imperatrice Eudoxia nel 402-407 per l'erigenda cattedrale di Gaza. ${ }^{83}$

Quasi sempre i capitelli hanno svolto un ruolo del tutto speciale e prioritario negli studi dedicati alla scultura architettonica costantinopolitana, mentre altri elementi non hanno suscitato pari attenzione, sebbene possano contribuire in modo altrettanto significativo a valutare i mutamenti intervenuti nella struttura e nel lessico decorativo, registrando il manifestarsi di nuove tendenze di gusto. Novità che tuttavia nel corso del V secolo comin-

\footnotetext{
${ }^{81}$ In proposito si veda Russo 2009b, 22-28, figg. 18-21 (con bibliografia precedente). Le divergenze formali rispetto ai capitelli di pilastro della Santa Sofia, evidenziate da Russo, senza tuttavia tener conto dell'incidenza delle notevoli differenze dimensionali, segnalerebbero a suo avviso una posteriorità dei capitelli della Porta d'Oro, datati al 425, poiché, scrive, la «mobilità delle superfici delle foglie a port'Aurea rispetto alla maggiore rigidità di quelle di S. Sofia va interpretata come segno di posteriorità, come una riproposizione se non un riflusso manierista rispetto alla novità sconvolgente che si manifesta nelle sculture della cattedrale con sguardo rivolto al futuro, pur nel percorso del medesimo alveo e nell'adozione degli stilemi presenti a S. Sofia» (ibidem, 27). Lo stesso dettaglio si nota anche sui capitelli dei pilastri con semicolonne dalla Rotonda del Myrelaion, datati al primo decennio del V secolo, cinque provenienti dallo scavo del 1931, oggi nel lapidario della Santa Sofia (inv. 135-139), il sesto scoperto invece nel corso delle indagini del 1961, è collocato all'esterno del Museo Archeologico, sul lato destro di Osmanbey Yokusu che sale verso il primo cortile del Topkap1 Saray1, cfr. da ultimo Russo 2007a, 11, figg. 49-53; 2009b, 28-29, fig. 34, con bibliografia precedente. I capitelli sono caratterizzati da un disomogeneo stato di lavorazione, alcuni sono semplicemente sgrossati, altri mostrano un decoro appena rifinito, mentre altri ancora esibiscono le foglie di acanto ritagliate a giorno con esperta manualità. Provengono dallo stesso scavo anche due mensole decorate con foglie di acanto i cui orbicoli sono caratterizzati ugualmente dalla stessa solcatura.

${ }^{82}$ Del ritrovamento è stata data notizia dal giornale on-line Haaretz il 5.4.2015 (Remnants from Ancient Church unscavated in Gaza by Constructions Workers).

${ }^{83}$ Marco diacono, Vita di Porfirio, cap. 84, cfr. Mango 1972, 31-32.
} 
ciano ad apparire, ma assai timidamente, nell'ambito di un contesto documentario ancora dominato da una sintassi decorativa di tradizione classica, lasciando affiorare i segni di una progressiva metamorfosi e il concretizzarsi di uno stile autonomamente costantinopolitano.

Paradigmatico al riguardo appare il percorso evolutivo delle cornici con mensole (o modiglioni), che in un certo qual senso costituiscono una sorta di fil rouge che collega gli episodi presi in esame, le cui modifiche più sensibili si rilevano nell'aggetto viepiù contratto delle mensole che ne attenua la simulata funzione portante, quasi da trasformarle in semplici componenti decorative. Numerosi sono gli esempi costantinopolitani di V secolo, mai sistematicamente censiti, e tutti quelli che hanno preservato l'angolo sono provvisti di una mensola diagonale. Quasi certamente aveva una mensola diagonale anche un meno noto frammento recuperato nelle rovine della ormai scomparsa chiesa di San Giovanni Battista, edificata nel sobborgo dell'Hebdomon, probabilmente al tempo di Teodosio I (FIG. 25), che appare assai vicina al modello sintattico classicheggiante delle cornici della Santa Sofia teodosiana. $^{84}$

Proviene ugualmente dall'Hebdomon, forse dal medesimo edificio, anche un frammento di trabeazione (FIG. 26), attualmente collocato nel giardino del Museo Archeologico di Istanbul, ${ }^{85}$ del quale è da notare l'inconsueto decoro sulla sima che riproduce in modo schematico, quasi grafico, un fregio di foglie, verosimilmente d'acqua, la cui lavorazione parrebbe solo in parte completata. ${ }^{86}$ Accuratamente rifinito è invece il fregio di foglie d'acqua sulla sima di un frammento angolare di trabeazione, proveniente da Costantinopoli, ed esattamente dalla Yedikule, oggi nei Musei di Berlino, datata, ricordo, al V secolo ${ }^{87}$ (FIG. 27).

Alla medesima epoca può essere attribuito un colossale elemento di trabeazione scoperto nel 1960 nell'area di Saraçhane, presso la Şehzade Camii, ${ }^{88}$ purtroppo non più ispezionabile perché completamente interrato. Esso presentava una sequenza di grandi palmette tra loro raccordate da una sottile fascia orizzontale desinente in spirali, aderenti al piano di fondo della sima (FIG. 28). I medesimi stilemi caratterizzano anche le palmette scolpite a bassissimo rilievo su una serie d'imposte recuperate nella stessa area urbana e quasi certamente provenienti dal medesimo edificio. ${ }^{89}$

\footnotetext{
${ }^{84}$ DAI Istanbul, Neg. Nr. R317-3189. Cfr. Kleiss 1974, 211-213, fig. 45; Niewöhner 2009, 144; Niewöhner 2017, 303 304, figg. 148-149. Malgrado sia fratturato proprio in corrispondenza dell'angolo è ipotizzabile la presenza di una mensola diagonale.

${ }^{85}$ Inv. 3206: Barsanti 1989, fig. 136; Niewöhner 2009. 144-147, fig. 1-3. Nei soffitti del frammento, riutilizzato come frantoio, trovano posto elementi vegetali.

${ }^{86}$ Anche il decoro grosso modo analogo che caratterizza la sima di un altro frammento di trabeazione all'esterno dello stesso Museo non sembra rifinito.

${ }^{87}$ Wullf, Volbach 1923, 8, n. J6716. Il fregio, di tipo poco usuale, è caratterizzato da foglie d'acqua disposte su due registri, di cui, quelle in primo piano, percorse da profonde solcature, sono tra loro unite alla base a guisa di festone; tra di esse affiora la costolatura delle foglie in secondo piano; una foglia d'acanto ne decora gli angoli. Il miglior raffronto è rappresentato dalle imposte in opera nel battistero degli Ortodossi a Ravenna, datate al terzo quarto del V secolo, cfr. Sodini 1977, 101, fig. 55.

${ }^{88}$ Lafontaine 1959-60, 359, tav. I, 1. La foto di P. Steyer, qui pubblicata è dell'archivio DAI di Istanbul, Neg. Nr. K62747.

${ }^{89}$ Le imposte provengono dal distrutto complesso dell’Ibrahim Paşa Hamami, cfr. Barsanti 1982, figg. 1-3; Zollt 1994, 56-57, cat. 121-130, tav. 28. Imposte analoghe sono state trovate nell'area della Santa Eufemia. mentre altre si coordinano ai capitelli dei già ricordati pilastri doppi del Myrelaion.
} 
Quasi del tutto invisibile è pure la trabeazione in opera nel nartece dell'unico edificio costantinopolitano superstite di V secolo, la basilica di San Giovanni di Studio, ormai da tempo in condizioni di drammatico degrado e quasi del tutto celata da piante rampicanti ${ }^{90}$ (FIG. 29), tant'è vero che solo a malapena s'intravedono le mensole e gl'inserti decorativi nei lacunari, nei quali si possono comunque distinguere elementi vegetali ed anche figure animali di specie diverse. ${ }^{91}$

La trabeazione poggia su splendidi capitelli compositi con foglie di acanto finemente dentellato, un tipo di capitello di tradizione microasiatica rielaborato dagli artefici del Prokonnesos, che d'allora s'impose come grande protagonista della scultura architettonica costantinopolitana, almeno fino alla prima metà del VI secolo, la cui eccezionale diffusione è documentata da centinaia di esemplari distribuiti in una vastissima area geografica, i quali ne riproducono tuttavia quasi sempre in forma più o meno semplificata l'elaborato apparato decorativo. ${ }^{92}$

Una provenienza dall'area costantinopolitana è stata suggerita, forse non a torto, anche per un imponente architrave di porta di marmo proconnesio, caratterizzato anch'esso da mensole diagonali, trovato nella basilica 'cupolata' di Meriamlik in Cilicia, oggi nel Museo Archeologico di Adana (FIG. 30), il quale venne con ogni probabilità importato assieme ad altri elementi marmorei per l'arredo architettonico e liturgico dell'edificio datato agli ultimi anni del V secolo. ${ }^{93}$

Tornando a Costantinopoli, un breve cenno infine alle cornici riutilizzate in due dei quattro piloni di rinforzo, addossati alla fronte occidentale della Santa Sofia, ${ }^{94}$ la cui sima decorata da un fregio di palmette è stata giustamente avvicinata a quella delle cornici provenienti dallo scavo della Santa Eufemia all'Ippodromo, datate tra la fine del V e l'inizio del VI secolo. ${ }^{95}$

Proprio nei primi anni del VI secolo la secolare parabola della classicità i cui stilemi, talora quasi sfibrati, caratterizzavano ancora quelle cornici, si conclude in modo repentino, quasi senza avvisaglie, nel momento in cui prese forma il progetto decorativo ideato per il San Polieucto, l'ambiziosa fondazione della nobile Anicia Giuliana. ${ }^{96}$ Gli ideatori del

\footnotetext{
${ }^{90}$ Deichmann 1956, 69-72, figg.17-18; Mathews 1976, figg. 15-2,3, 15-20, 22,24; Butler 1990, 87-91, fig. 28; Milella 1996, 66, fig. 5; Niewöhner 2017, 269-270, figg. 67-69.

${ }^{91}$ A stento si possono riconoscere, assieme a elementi vegetali di vario tipo un'aquila di prospetto, un orso, un piccolo drago e alcuni volatili.

${ }^{92}$ Per la diffusione della tipologia, si veda, da ultima, Guiglia 2011(2015), 132-133, con ampia bibliografia.

${ }^{93}$ Herzfeld, Guyer 1930, 49-52, figg. 50-51; Deichmann 1956, 88-89; Butler 1990, 92-93; Niewöhner 2017, 279-280, figg. 92-94. Sono invece di manifattura locale le cornici di entrambe le chiese di Alahan Monastir, ugualmente datate al maturo V secolo, tra le quali merita di essere ricordato l'architrave del 'Portale degli Evangelisti' della chiesa ovest, caratterizzato da un esuberante decoro vegetale e zoomorfo e da mensole diagonali: Gough 1985, tavv. 10, 16-18; Butler 1989, 93-95, fig. 33.

${ }^{94}$ Schneider 1941, 31-33; Barsanti, Guiglia 2018.

${ }^{95}$ Naumann, Belting 1966, 81-82, n. 4c-d, tavv.14, 48f; Barsanti, Guiglia Guidobaldi 1996, 84, figg. 6-8; Flaminio 2004, 647-648; Niewöhner 2017, pp. 264-266, figg. 55-56. Più recentemente, sono state prese in esame da Alessandra Guiglia Guidobaldi e da chi scrive nell'ambito di un'ampia indagine sul fenomeno del reimpiego nella Santa Sofia (Barsanti, Guiglia 2018).

${ }^{96}$ Sugli scavi e i ritrovamenti del San Polieucto si veda anche la mostra fotografica From the Bosporus to Oxford: Unseen Photographs from Prof. Harrison's Byzantine Excavations in Istanbul. An Exhibition at the Ioannou Centre for Classical and Byzantine Studies, University of Oxford, January-March 2011, a cura di G. Parpulov e L. Schachner. Per la
} 
progetto attinsero infatti a un bagaglio di conoscenze di matrice prevalentemente sasanide che contribuì alla formulazione di un linguaggio estraneo prima d'allora alla cultura artistica costantinopolitana, determinando una svolta decisiva, un vero e proprio netto spartiacque, che è stato non a torto più volte definito un episodio di portata 'rivoluzionaria', con il quale si dischiuse una nuova era artistica. ${ }^{97}$

Le splendide sculture emerse dallo scavo del San Polieucto hanno infatti rivelato l'incredibile vitalità creativa dei loro artefici i quali seppero coniugare con grande versatilità e straordinaria maestria esecutiva stilemi naturalistici di tradizione classica e temi decorativi ispirati al linguaggio decorativo della Persia sasanide, e, paradigmatico al riguardo, può essere anche l'esempio offerto dalle cornici con mensole. ${ }^{98}$

Le cornici, con funzione di architravi di porta, sono di due tipi, entrambe con fragili monogrammi ritagliati a giorno nei piccoli soffitti, sulle quali la sintassi decorativa 'canonica' viene totalmente sconvolta. L'ornamentazione ha il sopravvento, mutando l'articolazione e i tradizionali rapporti dei piani prospettici, palesando un gusto per formule decorative caratterizzate da fantasiose forme vegetali ibridate (FIG. 31). Uno stesso gusto sintattico caratterizza l'ornamentazione dell'altra cornice (FIG. 32) che presenta anche un fregio con coppie di cornucopie allacciate alla base, dalle quali si generano tralci vegetali, un tema di sapore classico, tra l'altro onnipresente nel lessico decorativo, quasi un elemento topico, variamente declinato nel sistema ornamentale della contemporanea scultura architettonica costantinopolitana. ${ }^{99}$ Ed è proprio nel fertile alveo di questa innovativa concezione dell'ornato che si concretizzò anche la creazione dei capitelli imposta, il cui tessuto decorativo avvolgente annullò la plastica articolazione in zone dei capitelli classici. ${ }^{100}$

Echi di quella straordinaria euforia creativa si ritrovano, ma in forma più pacata, a distanza di pochi anni nei Santi Sergio e Bacco. ${ }^{101}$ Nel progetto decorativo dell'edificio, messe da parte le più estrose creazioni del San Polieucto venne recuperata, da un lato, con una certa fedeltà, una sintassi compositiva di tradizione classica per la superba trabeazione che scandisce in due piani lo spazio interno dell'edificio, sulla quale si dispiega la

\footnotetext{
datazione dell'edificio, circoscritta entro il primo quarto del VI e conclusasi con tutta probabilità nel 522, cfr. Russo 2012, 101, 103 e nota 9.

${ }^{97}$ Sulle innovative esperienze creative degli scultori-decoratori del San Polieucto è stato posto ampiamente l'accento da: Mango 1977; Strube 1984; Harrison 1986; Harrison 1989; Barsanti 1998, 316-317; Guiglia Guidobaldi 1998, 337-338; Pasquini Vecchi 1999; Russo 2004; Russo 2006b; Russo 2007b; Russo 2008; 73-77, 78, figg. 50-54; Barsanti, Pilutti Namer 2009, 139-140.

${ }^{98}$ Strube 1984, 70-77, figg. 70-71; Harrison 1986, cat. 2a e 2b, 121, fig. C e figg. 111-113; Harrison 1989, 90-91, figg. 100, 103-105; Butler 1990, 96-97, fig. 34; Guiglia Guidobaldi 1998, 337, fig. 28; Pasquini Vecchi 1999, 121 -122, figg. 6-7; Russo 2004, 753, figg. 23-24; Niewöhner 2017, 283-285, figg. 105-107.

${ }^{99}$ Questo tema esaugurante è stato variamente e ampiamente declinato nel repertorio costantinopolitano, come mostra, ad esempio, l'arredo plastico della Santa Sofia, secondo moduli e schemi replicati nella basilica B di Filippi, cfr. Guiglia Guidobaldi 1998, 339-340, fig. 29-30.

${ }^{100}$ Una creazione che configura, osserva Jean-Pierre Sodini $(2000,435)$ : «un'evolution aussi forte que l'introduction du chapiteau corinthien dans l'architecture grecque». Ne offrono splendida testimonianza le multiformi trame decorative dei fragili involucri lavorati a giorno sul kalathos di una vasta gamma di capitelli, anche del così detto tipo 'a pannelli': Barsanti 1998, 316, 321; Barsanti 2006.

${ }^{101}$ Van Millingen 1912, 62-83; Ebersolt, Thiers 1913, 21-51; Deichmann 1956, 72-76; Müller-Wiener 1977, 177-185; Guiglia Guidobaldi 1998; Croke 2006; Russo 2008, 82, 87, 90, figg. 51, $63-64$.
} 
magniloquente iscrizione dedicatoria di Giustiniano e Teodora ${ }^{102}$ (FIGG. 33-34), mentre dall'altro non si esita a proporre nuove creazioni, rappresentate soprattutto dai capitelli polilobati i quali, su una struttura assolutamente innovativa di plastica movimentazione, esibiscono i monogrammi imperiali ${ }^{103}$ inseriti in una delicata trina vegetale magistralmente ritagliata a giorno ${ }^{104}$ (FIG. 35). Altrettanto originale è la elaborata trama ornamentale lavorata a giorno sulla coppia di capitelli a imposta in opera nella trifora sud (FIG. 36). Entrambi recano su entrambi i lati un monogramma, a sinistra, del nome di Giustiniano, mentre l'altro, a destra, del titolo BACI $\Lambda \mathrm{E} \Omega \mathrm{C}$. $^{105}$ Ad essi corrisponde la coppia di capitelli, pressoché identici, in opera nella opposta trifora nord, riapparsi nel corso del recente restauro dell'edificio, ma dei quali è visibile solo la porzione che affiora dalla parete (FIG. 37).

La fitta decorazione di questi capitelli comprende, combinati con grande fantasia, cornucopie, tralci vegetali, foglie di varia natura e motivi geometrici, un dettaglio quest'ultimo che sembra quasi prefigurare timidamente le più complesse griglie geometriche, anche asimmetriche, coordinate ad elementi vegetali, ritagliate, ad esempio, sui piccoli capitelli in opera nella Santa Sofia sui pilastrini di verde di Tessaglia della loggia imperiale ${ }^{106}$ e, soprattutto, sulle imposte in opera nei grandi finestrati delle gallerie. ${ }^{107}$ Il singolare motivo delle due cornucopie sovrapposte, dalle quali si espandono tralci vegetali, è invece una puntuale citazione del repertorio del San Polieucto, ed esattamente delle candelabre di cornucopie protagoniste del decoro ritagliato a giorno sul kalathos dei grandi capitelli provenienti appunto da quella chiesa, ma ormai da lungo tempo in esilio a Barcellona e a Venezia. $^{108}$

Innovativi sono pure i sedici capitelli ionici ad imposta in opera nella galleria dei Santi Sergio e Bacco (FIG. 38), nei quali la canonica struttura con balustri rettilinei viene modificata dalla disposizione in diagonale delle volute che sembrano in tal modo generare un effetto quasi rotante. ${ }^{109}$ Le forme vegetali più o meno ibridate che ne rivestono uniformemente l'imposta sono lavorate a giorno, ma solo sul lato rivolto verso il naos (FIG. 39a), dove campeggia il monogramma imperiale incorniciato da una coppia di mezze foglie

\footnotetext{
${ }^{102}$ Per i dodici esametri che compongono l'epigramma celebrativo dell'evergetismo imperiale, cfr. Feissel 2000, 89; Croke 2006, 46-53. Gli esametri sono scanditi da foglie cuoriformi con lungo stelo ondulato; per lo stile delle eleganti lettere maiuscole, di regolare forma tondeggiante, alte circa $20 \mathrm{~cm}$, ritagliate con leggero risalto sul piano di fondo, cfr. Mango 1991, 244-245.

${ }^{103}$ In proposito, cfr. Swainson 1895; Van Millingen 1912, 73-74.

${ }^{104}$ Guiglia Guidobaldi 2007, 119; Brüx 2008; Russo 2006b; Russo 2012, 136-148, figg. 57-58; Barsanti 2017 a, 47 dove si ricorda che i primi a definire 'a melone' i capitelli in questione, in particolare i due esemplari in opera nell'atrio del battistero della Santa Sofia, sono stati Lethaby e Swainson 1894, 252, 257, fig. 54.

${ }^{105}$ Ebersolt, Thiers 1913, 43-44, fig. 21; Zollt 1994, 84-85, cat. 207-208, tav. 34; Barsanti 1998, 330; Russo 2012, 139, fig. 61. Il decoro della coppia della trifora sud è interrotto su due lati opposti da fasce lisce, funzionali evidentemente all'appoggio di un telaio di finestra o d'incorniciatura di porta.

${ }^{106}$ Il primo e il quarto da nord sono di nuova fattura, copie eseguite in stucco, durante i grandi restauri ottocenteschi dei fratelli Gaspare e Giuseppe Fossati, cfr. della Valle 2004, 777-779, figg. 461-464; Russo 2012, 95, fig. 12.

${ }^{107}$ Guiglia Guidobaldi 1990, 280-286; Barsanti 1998, 334; Russo 2012, 95, figg. 6 e 14.

${ }^{108}$ Barsanti, Pilutti Namer 2009.

${ }^{109}$ Ebersolt, Thiers 1913, 44-46, figg. 22-24; Mathews 1976, figg. 29-26, 29-27; Zollt 1994, 20-21, cat. 26 , tav. 9 (con bibliografia); Barsanti 1998, 327-328; Guiglia Guidobaldi 2007, 36; Barsanti 2009, 130; Russo 2012, 139-140, 158, figg. 59-60.
} 
curvilinee concentriche, un altro motivo che trae spunto dal ricco repertorio del San Polieucto. ${ }^{110}$

Ai lati del monogramma si dispongono calici vegetali, arricchiti alla base da mezze foglie aperte ad ali e dai quali si espandono, in alto, rami vegetali. Sugli altri tre lati viene replicata la medesima composizione, ma non è lavorata 'a giorno' (FIG. 39b-c), bensì ritagliata con breve risalto dal piano di fondo, mentre al posto dei monogrammi vi sono piccoli tralci, foglie e corolle floreali, con un effetto d'insieme che dobbiamo comunque immaginare esaltato da una sfoglia d'oro che ne ricopriva quasi certamente la superficie scolpita, un effetto evocato forse dalle parole di Procopio quando descrive l'edificio tutto splendente di marmi e d'oro, alludendo non solo ai mosaici. ${ }^{111}$ All'epoca, la doratura e la policromia degli elementi architettonici, ereditata dalla passata classicità, era una pratica comune, largamente utilizzata, come testimoniano infatti vari episodi accertati nella stessa capitale ${ }^{112}$ e anche in più di una regione dell'Impero. ${ }^{113}$

All'indubbio successo riscosso dai capitelli polilobati, di cui sono stati censiti all'incirca un centinaio di esemplari, disseminati nell'ambito di un vasto contesto geografico e nei quali viene replicata con molteplici varianti morfologiche, ma in versioni per lo più ridotte $\mathrm{e}$ semplificate, la complessità della trama vegetale del prestigioso modello creato per la fondazione imperiale, ${ }^{114}$ fa riscontro una diffusione decisamente limitata e circoscritta all'area costantinopolitana dei capitelli ionici ad imposta nell'innovativa variante con volute diagonali, le cui attestazioni, vorrei soggiungere, sono per lo più collegabili ad edifici di fondazione giustinianea, primi tra tutti, la splendida serie di capitelli in opera nelle gallerie della Santa Sofia. ${ }^{115}$

\footnotetext{
${ }^{110}$ Ad esempio, le forme vegetali ritagliate sul già citato architrave con mensole, immediatamente sotto il fregio con le cornucopie. Nei capitelli variano unicamente gli ornati che si alternano sulle volute: fogli e tralci di vario tipo.

${ }^{111}$ Procopio, De aedificiis, IV, 1-7: Barsanti 2016, 64.

${ }^{112}$ In proposito Barsanti 2016. Resti di policromia sono stati accertati sulle sculture del San Polieucto, così come estese tracce di colore azzurro sono ancora visibili nel sottofondo dei decori lavorati a giorno sui rivestimenti del naos della Santa Sofia, mentre i capitelli della basilica A di Beyazit, al momento della scoperta, mostravano ancora resti di sfoglia d'oro e tracce di azzurro nello sfondo (Mathews 1976, 28).

${ }^{113}$ Tra le testimonianze più significative ricordo i piccoli capitelli corinzi della basilica di Mitropolis a Gortina di Creta che hanno eccezionalmente preservato sulla superficie scolpita alcune sottili sfoglie d'oro ed anche tracce dello strato preparatorio di colore rosso, cfr. Baldini Lippolis 2013, 34, fig. 6a-b; Barsanti 2016, 66.

114 Guiglia Guidobaldi 1995, 605-609; Brüx 2008, 255-274; Russo 2012; Barsanti 2017a, dove, al corpus dei capitelli catalogati dal Brüx, oltre all'esemplare di Qasr Hallabat (Dennert 2010, 201), si aggiungono: un bel capitello nel giardino (Feyaman Duran evi bahçesi) del Rettorato dell'Università di Istanbul, un altro, in condizioni molto frammentarie, nel Museo Archeologico di Çorum, forse dalla vicina Euchaita, un capitello nel Museo di Silifke e, infine, un capitello di notevoli dimensioni che si trova nel cortile della piccola moschea di Kasim Çavuş Camii a Eyyup.

115 Agli esemplari presi in esame da Zollt 1994, 22-25, 324-330, cat. 30-35; Guiglia Guidobaldi 2007, 36-38; Guiglia Guidobaldi 2010, 123-124, si può aggiungere un singolare capitello, di notevoli dimensioni, trovato in prossimità della punta del Serraglio, caratterizzato da una voluminosa imposta modulata da piani concavi ed emergenze convesse, scand iti orizzontalmente da una serie di listelli che si sovrappongono in ritmica successione (Barsanti 1998, 328). Non va peraltro tralasciata l'ancor più rara variante, con volute angolari, vale a dire disposte sotto gli spigoli dell'imposta, più vicina ai modelli classici, i cui antefatti microasiatici sono stati messi in evidenza da Russo, in rapporto agli esemplari del Ginnasio di Sardi e, soprattutto, del San Giovanni di Efeso (Russo 1999, 41-42, figg. 33, 36-37, 40; Guiglia Guidobaldi 2007, 44 e nota 45; Russo 2007b). Resta comunque da approfondire la diffusione di questa variante in ambito costantinopolitano, finora documentata da soli tre esemplari (Zollt 1994, 20, cat. 24-25, tav. 8; Russo 1999, 41, fig. 32). Un'indagine al riguardo potrebbe in effetti rivelarsi densa d'implicazioni, anche in considerazione di una più tarda ripre-
} 
In occasione del recente restauro dei Santi Sergio e Bacco, ultimato nel 2006, è stato rimosso lo strato di calce che ricopriva le fragili trame lavorate a giorno dei capitelli polilobati, dei capitelli imposta e degli ionici ad imposta, nonché la superficie scolpita della trabeazione che si articola in tratti rettilinei e curvilinei, mettendo in evidenza molti interessanti dettagli relativi ai sistemi di lavorazione e di rifinitura, con ogni probabilità realizzata in larga parte dopo la messa in opera, come suggeriscono le porzioni dell'intaglio a giorno non finito, talora solo abbozzato. Si sono potute inoltre ricavare molte informazioni sulle modalità dell'assemblaggio dei 29 segmenti che compongono la trabeazione, ${ }^{116}$ così come sono ritornati in vista i segni di seriori interventi di restauro (FIG. 40) e ampi resti di policromia sul lato nord, ma di epoca verosimilmente ottomana. ${ }^{117}$

Per quanto riguarda specificatamente il sistema di assemblaggio sono riapparse diverse irregolarità nelle connessioni tra i blocchi, opportunamente coincidenti con i capitelli e i pilastri (FIG. 41). Si notano infatti aggiustamenti talora approssimativi nel raccordo della sequenza decorativa, così come varie lettere dell'iscrizione risultano tagliate a metà proprio in corrispondenza delle giunture (cfr. FIG. 37), il che farebbe pensare che la lavorazione del pezzo, compreso il fregio epigrafico, fosse stata effettuata, almeno in parte, prima della messa in opera, per essere completata nei dettagli una volta collocato in situ, come lasciano appunto supporre, anche in questo caso, alcuni dettagli dei fregi 'non finiti'.

Come si è detto, la trabeazione appare legata alla tradizione classica, pur se con alcune sensibili modifiche, rilevabili sia nell'allineamento verticale dei piani decorati, giustapposti senza tener conto delle reciproche relazioni, sia nella sensibile contrazione dei modiglioni, rispetto ai quali predomina l'aggetto del sottostante fregio di girali d'acanto, riassunto in forma di toro a sezione accentuatamente convessa, quasi circolare, e privato di ogni effetto chiaroscurale da una raffinata lavorazione a giorno ${ }^{118}$ (cfr. FIG. 40) Quand'anche sia stato recuperato un lessico tradizionale, come il kyma ionico che incornicia le mensole, tra cui, ben evidenti, sono quelle pentagonali ad andamento diagonale in corrispondenza degli angoli (FIG. 42), non mancano qualche significativa novità e i segni di una matrice di gusto che rinvia anche in questo caso al vocabolario ornamentale del San Polieucto, novità apprezzabili sia nel fregio sulla sima, con elementi fitomorfi aperti ad ali, sia nelle ibridi figure vegetali che hanno preso il posto delle foglie di acanto sotto le mensole. Nei soffitti trova posto una serie di ornati prevalentemente vegetali, tralci, calici fogliari e girandole, ${ }^{119}$ ma anche (FIG. 43), dischi, pesci, assieme ad un singolare oggetto, che compare pure su due soffitti di finestra nella galleria della Santa Sofia e su un pilastrino della recinzione presbiteriale di VI secolo della basilica romana di San Clemente a Roma. ${ }^{120}$ Nell'oggetto, variamente inter-

\footnotetext{
sa negli esemplari di XI secolo in opera nel San Marco di Venezia, il cui modello di riferimento sarebbe in verità seducente poter collegare alla fabbrica giustinianea dell' Apostoleion (Barsanti, Pedone in c.d.s.).

${ }^{116}$ Butler 1990, 98-99, figg. 21, 28; Butler 1992, 66-67, fig. 41.

${ }^{117}$ Le cromie che ricoprivano la superfice della scultura architettonica dell'edificio sono documentate anche dai disegni di Chrles Maria Texier, cfr. Pedone 2013, 1675, fig. 7.

${ }^{118}$ Ebersolt, Thiers 1913, 47-48, figg. 19-20, 25; Mathews 1976, figg. 29.15, 29.28, 29.30. Vedi ora Niewöhner 2017, 294-295, figg. 128-129.

${ }^{119}$ Cfr. l'esigua selezione di ornati pubblicati da Pulgher 1878, tav. III, 2.

${ }^{120}$ Barsanti, Flaminio, Guiglia Guidobaldi 2015, cat. 86, 235-237, tav. L. Lo stesso oggetto compare anche nel soffitto di un frammento di cornice con mensole reimpiegato nella diruta chiesa del monastero di San Giovanni Teologo a Pelekete/Zeytinbağı, in Bitinia, datata tra la fine del V e l'inizio del VI secolo, cfr. Mango, Ševčenko 1973, 248, figg. 44-45, 47-48; Ötüken 1996, 171-173, cat. M 35a, tav. 28,1.
} 
pretato, è stato riconosciuto sia un piccolo recipiente di uso liturgico, sia una sorta di pane sacro, sia piuttosto una suppellettile da tavola. ${ }^{121}$ Il decoro della trabeazione si completa nei ventuno soffitti tra le coppie di colonne e i pilastri, i quali si articolano in doppi o singoli riquadri, rettilinei o curvilinei in corrispondenza delle esedre, con tipologie decorative caratterizzate da schemi geometrici e ornati analoghi a quelli dei plutei contemporanei ${ }^{122}$ (cfr. FIGG. 35-36).

Nel corso dei lavori di restauro, dei quali manca purtroppo una circostanziata relazione finale, sono state condotte anche alcune indagini archeologiche all'esterno e all'interno del monumento che hanno portato alla scoperta, davvero eccezionale, di una piccola cripta cruciforme nell'area presbiteriale (FIG. 44) che appare assai simile alle cripte delle basiliche di San Giovanni di Studio e della Theotokos Chalkoprateia, entrambe di V secolo. ${ }^{123}$ Come quelle, le pareti del piccolo vano cruciforme, accessibile dal lato est, erano rivestite da lastre marmoree. $^{124}$

Non meno importante è stata la rimozione del pavimento che ha riportato in vista le basi delle colonne, di tipo attico su plinto ottagonale, ${ }^{125}$ alcune delle quali recano incisa una sigla di lavorazione. ${ }^{126}$ A una profondità di circa mezzo metro sono stati inoltre individuati ampi tratti dell'originaria pavimentazione a grandi lastre marmoree, molte di reimpiego, come, ad esempio, una lastra di grandi dimensioni, alta circa $2 \mathrm{~m}^{127}$ (FIG. 45), trovata in condizioni frammentarie nella zona centrale del pavimento, decorata con una grande croce latina su globo, databile alla prima metà del VI secolo, ${ }^{128}$ mentre sembrerebbe più tarda un'altra lastra che reca incisa una croce a estremità patenti che si erge sul monte Calvario. Sono stati recuperati molti altri frammenti di rivestimenti parietali e di soffitti di VI secolo, ${ }^{129}$ riutilizzati anche nel pavimento del portico ottomano, da dove è stato rimosso un pluteo quadrangolare caratterizzato da uno schema decorativo non particolarmente diffuso, se non in alcuni plutei di epoca giustinianea. ${ }^{130}$

Non è improbabile che parte di questi marmi siano le spoglie della ormai scomparsa, contigua chiesa dei Santi Pietro e Paolo, ${ }^{131}$ mentre altri facevano forse parte dell'arredo

\footnotetext{
${ }^{121}$ Barsanti, Guiglia Guidobaldi, Flaminio 2015, 236-237.

${ }^{122}$ Cfr. Yalçin 2004, 257-289.

${ }^{123}$ Mathews 1971, 27, 32,33; Mathews 1976, 143, 319, figg. 15-27; Guidobaldi 1994.

${ }^{124}$ Le uniche notizie al riguardo sono state fornite dal giornale on-line Kent Arkeolojisi del 15 Nisan 2015: Little Hagia Sophia Mosque (Sergius and Bacchus) Excavations, figg. 21-22.

${ }^{125}$ La struttura delle basi di tipo attico, su plinto ottagonale, è simile a quella delle basi della chiesa di Sant'Eufemia all'Ippodromo: Naumann, Belting 1966, 56-57, 69-70, figg. 19-20, tavv. 7d. 9a, b,d; Mathews 1976, figg. 12-5.

${ }^{126}$ Per le sigle (A, AKC e Z $\omega$ ), già segnalate da Deichmann 1976, 217, presenti sulle basi e sull'imoscapo delle colonne, sia di proconnesio, sia di pavonazzetto, si veda Marsili 2014a, 28-29, fig. 30.

${ }^{127}$ Questi materiali si trovano nel deposito del Vakif, ospitato nel seicentesco complesso dell'Amcazade Hüseyin Paşa Kulliyesi, a Saraçhane.

${ }^{128}$ Il tema decorativo trova significativo riscontro nelle lastre in opera nel finestrone occidentale della Santa Sofia (Gu iglia Guidobaldi 2004, 305) e altre lastre variamente reimpiegate nel monumento (Flaminio 2004, 265-267).

${ }^{129}$ I frammenti dei soffitti presentano schemi e decori, anche articolati in due riquadri, assai vicini a quelli della trabe azione nella chiesa, nonché a quelli degli architravi in opera nei finestrati al piano delle gallerie della Santa Sofia, per i quali cfr. Yalçin 2004.

${ }^{130}$ Guiglia, Barsanti, Flaminio 2010, 367, fig. 10; Pedone 2015, 385, figg. 18-19.

${ }^{131}$ Non sembra ancora risolta la problematica collocazione della chiesa, edificata intorno al 520 nell'ambito del complesso del palazzo di Hormisdas, accanto alla quale, a breve distanza di tempo, venne costruita la chiesa dei Santi Sergio e Bacco; Mathews 1971, 42-47; Vignoli 1978; Kostenec 2005-2006; Croke 2006, 28-29.
} 
liturgico dei Santi Sergio e Bacco. Se si eccettuano i due segmenti di transenna riutilizzati nel minber ottomano ${ }^{132}$ e due pilastrini reimpiegati nella rampa scalare che conduce alle gallerie, null'altro sembra infatti sopravvivere degli arredi liturgici dell'edificio, evidentemente rimossi allorquando, nei primissimi anni del XVI secolo, la chiesa venne trasformata in moschea, la Küçük Ayasofya Camii, ${ }^{133}$ così come sono scomparsi, in un momento non meglio precisabile, i plutei che probabilmente dovevano fungere da parapetti al piano delle gallerie. La loro presenza verrebbe in qualche modo segnalata dagli incassi praticati nelle basi delle colonne, in cui sono attualmente alloggiate le moderne transenne lignee. Lascia tuttavia perplessi l'assenza di segni, tracce di fori o d'incassi, sui fusti delle colonne, evidentemente funzionali alla messa in opera di parapetti marmorei e dei relativi pilastrini.

La vicenda costantinopolitana della mensola diagonale sembra concludersi proprio nei Santi Sergio e Bacco, poiché, in modo del tutto inatteso, nelle cornici che su due livelli scandiscono (FIG. 46) i piani architettonici del maestoso naos della Santa Sofia giustinianea, venne recuperato il disegno sintattico tradizionale, privilegiando, con una scelta, oserei dire, tradizionalista, di gusto quasi retrospettivo, antiquario, il modello canonico con mensole tutte ortogonali e tutte decorate con una foglia di acanto ${ }^{134}$ (FIG. 47). Va comunque osservato quanto sia stato modificato sia lo spessore, sia l'aggetto plastico delle mensole, e non è un effetto ottico. Le mensole s'impennano infatti verso l'alto, il che ne attenua il significato strutturale e l'aspetto funzionale portante, peraltro mimetizzato dal raffinato decoro à jour che le riveste (FIGG. 48-49) e che sembra quasi riassorbirle nelle rabescanti trame che si distendono sulle pareti, un effetto che veniva certo accentuato dalla doratura sulle superfici scolpite. ${ }^{135}$ Questo recupero di sapore classicistico che parrebbe passato del tutto inosservato, anche al Butler il quale ha condotto un ampio e sistematico studio sulle cornici della Santa Sofia, analizzandone caratteristiche funzionali, strutturali, esecutive e decorative, meriterebbe forse maggiore attenzione, soprattutto ai fini di una migliore comprensione ed un approfondimento delle linee guida del progetto decorativo ideato per la grande chiesa costantinopolitana. ${ }^{136}$

Il nostro percorso termina comunque là dove era cominciato, vale a dire, nell'area di Beyazit, a breve distanza dall'arco del Foro di Teodosio.

Nei primi anni quaranta del secolo scorso, l'ampliamento verso Vezneciler Caddesi della Facoltà di Lettere dell'Università di Istanbul portò alla scoperta di un anonimo complesso di tre chiese, tra le quali, una basilica convenzionalmente denominata A. ${ }^{137}$ Nello scavo venne recuperato un gran numero di eterogenei elementi marmorei di arredo architettonico e

\footnotetext{
${ }^{132}$ Barsanti 2004, 513-514, figg. 287-288.

${ }^{133}$ La chiesa venne trasformata in moschea da Hüseyin Efendi, capo degli eunuchi dell'harem di Beyazit II, cfr. Kurımtayıf 2001, 20-24.

${ }^{134}$ Butler 1985; Butler 1990 e Butler 1992. Sulle cornici vedi pure Niewöhner 2017, 294-297, figg. 130-133.

${ }^{135}$ Butler (1990, 13-16) ricorda anche le testimonianze di Salzenberg (1854, 23), Swift (1940, 67), Mango e Hawkins (1965, 145, nota 59), i quali hanno avuto modo di osservare tracce di doratura su cornici e capitelli.

${ }^{136}$ Sull'argomento, cfr. le riflessioni di Russo 2011 e di Marsili 2016. Non ancora chiarita è, ad esempio, la genesi della singolare struttura dei cosiddetti Kessellkapitell che ad avviso di Russo $(2012,155)$ deriverebbe direttamente da quella degli esemplari del San Polieucto, oggi a Barcellona e Venezia. Al riguardo andrebbero forse presi in considerazione anche alcuni capitelli ionici di ambito africano, come un esemplare delle Terme del Foro di Khamissa in Algeria (Pens abene 1986, 425, fig. 56f), caratterizzato da un'articolazione strutturale in qualche modo simile a quella dei capitelli giustinianei di Santa Sofia.

${ }^{137}$ Mathews 1976, 28-35; Muller-Wiener 1977.
} 
liturgico, tra i quali anche i frammenti di alcuni capitelli che replicano fedelmente, ma con dati dimensionali leggermente inferiori, l'elaborata struttura dei Kesselkapitelle, creati per la Santa Sofia giustinianea, ${ }^{138}$ e anche vari frammenti di una cornice con mensole, analoga, ma in versione semplificata, alle cornici marcapiano di quel prestigioso edificio, ${ }^{139}$ le quali possono essere, forse, non a torto, considerate le ultime testimonianze di questa classica membratura architettonica in ambito costantinopolitano.

Se pure in qualche modo assimilabili dal punto di vista decorativo alle cornici della grande chiesa giustinianea, quelle della basilica A appaiono, come si è detto, notevolmente semplificate con modifiche intervenute soprattutto nella loro conformazione strutturale e prospettica (FIG. 50a-b). Lo spessore delle mensole, vale a dire il loro usuale risalto tridimensionale è ridotto ai minimi termini, quasi fosse riassorbito nel blocco, sebbene la loro presenza sia suggerita, direi meglio simulata dalle foglie di acanto modellate su un piano appena rilevato; le mensole si alternano a scomparti rettangolari che identificano i soffitti nei quali sono accolti motivi di carattere vegetale che in larga parte replicano il variegato repertorio degli ornati delle cornici della Santa Sofia, ma non la loro raffinata lavorazione a giorno. ${ }^{140}$

In conclusione mi sembra utile completare il quadro del decoro architettonico sin qui tratteggiato con una breve riflessione su una tendenza di gusto in contrasto con le esperienze decorative fin qui ricordate, una tendenza quasi reazionaria, che si manifesta in un momento non meglio precisabile, ma quasi certamente intorno al quarto decennio del VI secolo, con scelte che privilegiano forme sobrie ed essenziali, quasi austere, ben esemplificabili con l'elegante capitello dall'Hebdomon, il cui decoro si articola in semplici pannelli trapezoidali incorniciati da lisce modanature, che accolgono i monogrammi imperiali di Giustiniano e Teodora $^{141}$ (FIG. 51). Un tipo di capitello, vorrei soggiungere, che a Bisanzio, forse più di altri, ebbe un ampio e ininterrotto successo fino ad epoche relativamente tarde. ${ }^{142}$

C.B.

\footnotetext{
${ }^{138}$ Kramer 2006, 25-29, figg. 21-23; Guiglia, Barsanti, Paribeni, Yalçin 2007, 315-317, figg. 1-3, 5; Russo 2007b, 35-37, figg. 16-23; Russo 2012, 156-157, figg. 89-94. L'elaborata a struttura di questi capitelli è stata raramente replicata, e sempre in versione ridotta e semplificata, come nel caso della coppia reimpiegata nella basilica di San Marco, degli esemplari (quattro pressoché completi, più altri frammenti) dalla basilica di Arnithas a Rodi, reimpiegati nel Palazzo del Gran Maestro, dell'isolato esemplare in opera nel portico del giardino di casa Wiegand a Belino e degli esemplari realizzati nel materiale locale per la basilica B di Filippi in Macedonia, cfr. Kramer 2006, 30-43, figg. 3-9, 24-42.

${ }^{139}$ Firatli 1951, tav. Vc-d; Guiglia, Barsanti, Paribeni, Yalçin 2007, 317, figg. 7-8; Russo 2007b, 37, fig. 24. Un richiamo alle cornici della Santa Sofia è stato ovviamente suggerito anche per quelle a modiglioni della basilica B di Filippi.

${ }^{140}$ Cfr. Butler 1990, 127-135.

${ }^{141}$ Cfr. Barsanti 1989, 182.

${ }^{142}$ In proposito si veda Di Bello in c.d.s., con un'ampia rassegna di esempi.
} 
Con la menzione degli aspetti tecnici, presente nel titolo dell'intervento, intendiamo riferirci a tutta quella articolata catena di processi organizzativi, logistici oltre che meramente esecutivi, che hanno come esito finale la realizzazione del manufatto marmoreo. Per quanto mi compete percorrerò quindi, succintamente e in modo selettivo, le tappe di quella filiera che dall'estrazione in cava porta ai primi stadi di lavorazione del marmo, quindi al trasporto di ingenti carichi di manufatti e in conclusione alla loro rifinitura e messa in opera nel cantiere dell'edificio cui essi erano destinati. L'approccio selettivo mira a dar conto, in prima istanza, di alcune più recenti novità e acquisizioni su determinati aspetti dell'industria del marmo di età paleobizantina, lasciando sullo sfondo questioni più generali, già affrontate in passato in diversi contributi. ${ }^{143}$

In questo senso maggior spazio andrà assicurato non tanto alle fasi di estrazione e prima lavorazione dei marmi, per la conoscenza delle quali siamo ancora largamente debitori dei surveys condotti circa quarant'anni fa da Nuşın Asgari nelle cave antiche dell'isola di Prokonnesos, ora Marmara Adas1 (FIG. 52; cfr. FIG. 3), ${ }^{144}$ quanto piuttosto alle complesse operazioni connesse all'invio dei marmi verso le mete di destinazione o i luoghi deputati di raccolta, tramite la rete di trasporto navale. In questi ultimi anni lo studio del fenomeno del commercio e della distribuzione di marmi e materiali litici da costruzione in età imperiale ha conosciuto un nuovo straordinario impulso: ne fanno fede importanti monografie come quella di Ben Russell incentrata sulle strategie economiche del roman stone trade $^{145}$ e la notevole quantità di indagini archeologiche subacquee che hanno per oggetto i relitti di naves lapidariae. ${ }^{146}$ Va detto, a questo proposito, che la congruenza di questa definizione - che ci deriva dall'antichità essenzialmente per un motto di spirito nel Satyricon di Petronio - viene messa ora in discussione da alcuni studiosi i quali, per l'età romano imperiale, non ravvisano l'esistenza di imbarcazioni progettate e costruite in funzione del trasporto di materiali litici. ${ }^{147}$ Analoghi dubbi potrebbero avanzarsi per l'età bizantina, se teniamo per valida l'interpretazione di un passo del De Aedifciis di Procopio relativo all'uso promiscuo delle imbarcazioni che scaricavano nei depositi dell'isola di Tenedo il grano dell'annona proveniente dall'Egitto, ${ }^{148}$ pur se un'altra fonte bizantina, i Miracula sancti Georgii, sembra voglia alludere invece, con un oscuro termine greco $(\sigma \chi \varepsilon \delta 10 v)$, ad imbarcazioni e-spressamente realizza-

\footnotetext{
${ }^{143}$ Cfr. Barsanti 1989; Sodini 1989; Sodini 2000; Niewohner 2007; Greenhalgh 2009; Sodini, Barsanti, Guiglia 1998; Barsanti, Guiglia, Paribeni 2010; Marano 2014; Barsanti, Paribeni 2016; Marano 2016. Fondamentali restano gli studi di Bryan Ward Perkins raccolti con bibliografia aggiornata in Marble in Antiquity 1992.

${ }^{144}$ Asgari 1988; Asgari 1989; Asgari 1995; Asgari, Drew Bear 2002. Per lo sfruttamento delle cave di marmo docimio in Frigia vedi Niewöhner 2007; Niewöhner 2014.

${ }^{145}$ Russell 2013a. Con un gioco di parole l'opera è stata considerata una vera e propria milestone da John Clayton Fant nella sua recensione: cfr. Clayton Fant 2016.

${ }^{146}$ Russell 2013a; Russell 2013b, 332-341 per l'inventario aggiornato dei ritrovamenti di relitti con carichi lapidei databili dal II al VII secolo d.C., cui aggiungi almeno Beltrame, Lazzarini, Medaglia 2012; Medaglia, Beltrame, Lazzarini 2014.

${ }^{147}$ Petronio Satyr., CXVII. Cfr. Beltrame, Vittorio 2012, 141; Gianfrotta 2016, 350-354.

${ }^{148}$ De Aed. V 1, 10-11, cfr. Castagnino Berlinghieri, Paribeni 2011, 65. L'isola dovette avere un ruolo strategico per gli scambi commerciali sin dall'età classica, proprio come punto di snodo e di stazionamento dei collegamenti navali tra Mar di Marmara e Mare Egeo, pesantemente condizionati dalla circolazione dei venti lungo il Bosforo (cfr. Rutishauer 2001, 200-201).
} 
te per trasportare colonne destinate al santuario eretto a Lidda Diospolis in onore di Giorgio. ${ }^{149}$

Tornando alle più recenti attività di ricerca archeologica nei fondali marini, riveste ovviamente un ruolo di particolare importanza per il nostro discorso la ripresa dello scavo del relitto di Marzamemi II. Il celeberrimo relitto, ${ }^{150}$ col suo altrettanto celebre carico di marmi bizantini di VI secolo destinati ad un ignoto edificio di culto, era stato indagato e i suoi materiali erano stati in gran parte recuperati grazie ai pionieristici scavi (FIG. 53) condotti da Gehrard Kapitän negli anni '60 del secolo scorso. ${ }^{151}$ Dopo una stasi di diversi decenni, nel corso dei quali la ricca messe di capitelli, basi, frammenti di colonne, lastre di recinzione, elementi di ambone tratti dai fondali a largo di Capo Passero era rimasta melanconicamente depositata in altri fondali, ovvero quelli della Latomia del Paradiso a Siracusa (FIG. 54), qualcosa negli ultimi anni si è cominciato a muovere: da un lato la ripresa degli studi e l'avvio di saggi mirati di documentazione e restauro dei materiali marmorei provenienti dallo scavo Kapitän, sostenuti dalla Soprintendenza per i Beni Culturali di Siracusa; dall'altro l'inizio di una nuova campagna di surveys e ricerche archeologiche nell'area occupata dal relitto, dirette dalla Stanford University in collaborazione con la Soprintendenza del Mare Regione Siciliana. ${ }^{152}$ Proprio queste ultime indagini, condotte con ben altri mezzi tecnici e diagnostici rispetto a quelli di cui potevano disporre i pionieri dell'archeologia subacquea della metà del secolo scorso, ${ }^{153}$ promettono di ampliare sensibilmente le nostre conoscenze del relitto: già dalle primissime stagioni di scavo emerge ad esempio l'immagine di una imbarcazione che, in aggiunta al carico di marmi, trasportava un numero non indifferente di anfore di diverse tipologie e aree di fabbricazione (LR1, LR2, in misura minore LR4), tale da far ritenere che esse costituissero un carico supplementare, formato da contenitori residuali utilizzati in un contesto di commerci di media e piccola distanza. ${ }^{154}$ Altri materiali marmorei, in aggiunta a quelli recuperati o comunque individuati da Kapitän, sono stati trovati in un'area più ampia di quella circoscritta al relitto e tale particolare dislocazione viene spiegata dagli archeologi come effetto di eventi traumatici quali l'urto al momento del naufragio, l'azione violenta delle mareggiate nel corso dei secoli, il saltuario ricorso agli esplosivi nella pesca di frodo. ${ }^{155}$ Un altro fattore che potrebbe aver contribuito alla perturbazione dei materiali è però l'eventuale frequentazione di questi fondali al fine di recuperare i marmi ben prima della data ufficiale di scoperta del relitto di Marzamemi: ricercando in maniera autonoma entro distinti fondi d'archivio a Siracusa e a Roma, Elena Flavia Castagnino Berlinghieri ed io abbiamo trovato difatti prove esplicite che, per lo meno già a partire dal 1913, quello che sarebbe divenuto in seguito il byzantine church wreck era stato fortuitamente individuato; in quell'anno difatti Paolo Orsi, soprinten-

\footnotetext{
${ }^{149}$ Cfr. Barsanti 1989, 106; Castagnino Berlinghieri, Paribeni 2011, 65. Forse il termine può derivare dal toponimo di Schedia, il grande porto fluviale sul ramo canopico del delta del Nilo, da cui chiatte di grande capacità caricavano merci provenienti dall'entroterra egiziano e le trasportavano verso Alessandria; su questa stazione di dogana e di smistamento delle merci vedi ora Bergmann, Heinzelmann 2014.

${ }^{150}$ Per una sintesi aggiornata a prima dell'avvio della attuale campagna di scavi vedi Castagnino Berlinghieri 2017.

${ }^{151}$ Kapitän 1980 con bibliografia precedente.

152 Leidwanger, Bruno 2013; Leidwanger, Tusa 2015; Leidwanger 2016; Leidwanger, Tusa 2016; Leidwanger, Greene 2017.

${ }^{153}$ Castagnino Berlinghieri 2012.

${ }^{154}$ Leidwanger, Tusa 2016, 136 e 139; Leidwanger 2018, 346-347. Sulle caratteristiche dei commerci marittimi e sulla loro relazione con lo sviluppo economico dei centri urbani vedi Arnaud 2015.

${ }^{155}$ Leidwanger, Tusa 2016, 130.
} 
dente e direttore del R. Museo Archeologico di Siracusa, informava la Direzione Generale Antichità e Belle Arti che "in prossimità della borgata di Marzamemi, di fronte alla punta Bove Marino, alla distanza di un chilometro circa dalla punta medesima ed alla profondità di sei metri circa, trovansi vari fusti di colonne in gran parte monolitiche e, pare, anche alcuni capitelli". ${ }^{156}$ Venne progettata un'operazione di recupero di questi tesori mediante salpamento, poi non concretizzatasi per mancanza di un'adeguata copertura finanziaria; nuovi progetti di recupero delle "colonne di un imprecisabile metallo" vennero avanzati nel 1927 da ingenui pescatori che vagheggiavano antiche città sommerse e nel 1934 dal baldanzoso cavalier Antonio Di Lorenzo Granieri dei marchesi Castelluccio, il quale confidava che, una volta portati a terra, i marmi avrebbero potuto "facilmente per il Tevere essere portati a Roma e servire ad un maggior decoro nella costruzione dei sacrari della Casa del Fascio e della Mostra della Rivoluzione Fascista". ${ }^{157}$ In tutti i casi sopra menzionati questi tentativi fortunatamente non furono messi in atto, ma resta evidente che, quanto meno nella prima metà del Novecento (se non addirittura prima), l'ubicazione del relitto era ben nota a livello locale e quindi non si può escludere a priori che, prima dell'intervento di Kapitän, si siano verificate manomissioni e inquinamenti dell'area archeologica. Si spiegherebbe meglio in questo contesto anche la comparsa di materiali che si possono senz'altro riconoscere come pertinenti al carico della nave, ma che non figurano tra quelli recuperati nello scavo degli anni ' $60 .{ }^{158}$

Intorno a questi ultimi pure si sono accese di recente le luci dei riflettori, grazie a due mostre, tenutesi nel corso del 2016 prima ad Amsterdam ${ }^{159}$ e poi a Oxford, ${ }^{160}$ dedicate alle ricchezze del patrimonio archeologico subacqueo in Sicilia. Una sezione importante delle due mostre - soprattutto di quella oxoniense - ha esposto per la prima volta al pubblico una cospicua selezione di marmi del relitto di Marzamemi, opportunamente puliti e restaurati. Tenuto conto di quanto la prolungata immersione possa aver contribuito alla corrosione delle superfici - osservando ad esempio il fusto della colonna si può riconoscere quale fosse il lato coricato sul fondo da quello esposto all'aggressione ambientale - la visione ravvicinata dei pezzi consente di valutare con più agio fino a quale stadio i marmi erano stati lavorati prima del loro imbarco e quali dovessero essere le operazioni di rifinitura che li attendevano al loro arrivo a destinazione. Le basi delle colonne ad esempio (FIG. 55) appaiono in uno stato non completo di finitura, ${ }^{161}$ che però non ne avrebbe pregiudicato la messa in opera, dal momento che basi in marmo proconnesio analogamente trattate si trovano comunemente in varie località dell'impero ed anche nella stessa Costantinopoli. ${ }^{162}$ Nonostante i danni causati dalle con-

\footnotetext{
${ }^{156}$ Per i documenti conservati all'Archivio Centrale dello Stato vedi ACS, Min. P.I., AA.BB.AA., Div. I 1908-1924, Busta 185, fasc. 611, Siracusa 1913-1914 - Pachino. Scoperte di colonne antiche a Marzamemi nel mare. L'intero complesso di documenti è attualmente in corso di pubblicazione (Castagnino Berlinghieri, Paribeni in c.d.s.).

${ }^{157}$ ACS, Min. P.I., AA.BB.AA., Div. I 1908-1924, Busta 185, fasc. 611, Siracusa 1913-1914 - Pachino. Scoperte di colonne antiche a Marzamemi nel mare. Cfr. Castagnino Berlinghieri, Paribeni in c.d.s.

${ }^{158}$ Castagnino Berlinghieri, Guzzardi 2014.

159 Sicily and the Sea 2016, 127; La mostra si è tenuta presso l'Allard Pierson Museum di Amsterdam dall'ottobre del 2014 all'aprile del 2016.

${ }^{160}$ Storms, war \& shipwrecks. Treasures from the Sicilian Sea, Oxford, Ashmolean Museum 21 June - 25 September 2016.

${ }^{161}$ Il particolare viene sottolineato in Russo 2006, 245; Russo 2010, 38.

${ }^{162}$ Per una panoramica di esempi di questo tipo di basi non finite rinvio a Barsanti 2013b, 490-491, con bibliografia precedente. Sulle motivazioni di questa parziale lavorazione delle basi rinvio alle argomentazioni di segno economicoproduttivo avanzate da Jean-Pierre Sodini (cfr. Sodini, Kolokotsas 1984, 34) e alle osservazioni in merito in Russo 2010 ,
} 
crezioni calcaree i capitelli (FIG. 56), del tipo VII della classificazione del Kautzsch, mostrano uno stato di finitura pressoché completo ad eccezione della parte superiore del kalathos. ${ }^{163}$ Di contro le lastre per la recinzione presbiteriale (FIG. 57) appaiono in uno stato di esecuzione pressoché completa, così come gli elementi che componevano l'ambone a doppia rampa in verde antico di Tessaglia (FIG. 58).

I dati desumibili dalla visione ravvicinata collimano essenzialmente con quanto verificato da altri studiosi, come Eugenio Russo, che in passato hanno avuto modo di esaminare partitamente i marmi del relitto ${ }^{164} \mathrm{i}$ quali, rispetto alla dibattuta questione del loro grado di lavorazione più o meno compiuto, potrebbero definirsi come "passibili" di completamento; vale a dire come materiali già sufficientemente elaborati, negli ergasteria posti in prossimità delle cave o comunque ad esse collegati, per poter essere messi in opera, ma che necessitavano di interventi minimi per le operazioni di assemblaggio e conservavano inoltre margini di miglioramento nella definizione e nella finitura di alcuni particolari, che potevano essere eventualmente apportati a discrezione e per volontà del committente.

Il prosieguo delle indagini archeologiche subacquee porterà di certo, nel prossimo futuro, nuovi dati che potranno confermare o modificare le nostre opinioni sul relitto di Marzamemi; per questa ragione più che lasciarsi andare a valutazioni definitive, credo sia più costruttivo stilare un'agenda dei dati da validare e delle questioni nodali da sciogliere.

Una prima verifica è quella dell'effettiva provenienza di tutti i marmi bianchi dalle cave del Proconneso; per alcuni campioni prelevati dallo scavo in corso è stata già eseguita l'analisi degli isotopi stabili con risultati parziali compatibili con il proconnesio ma anche con altri litotipi, ${ }^{165}$ ragione per cui il range dei campioni andrebbe ampliato e sottoposto ad analisi incrociate di altro genere, come EPR (risonanza paramagnetica elettronica) e petrografia; ${ }^{166}$ ricordo che per complessi di marmi come quello delle due chiese di Latrun in Cirenaica (FIG. 59) - spesso accostati a vario titolo al set del relitto di Marzamemi - le recenti analisi condotte da Donato Attanasio hanno definitivamente chiarito che accanto a manufatti in proconnesio ve ne sono diversi in marmo tasio, in particolare tra le lastre di recinzione presbiteriale, secondo una ripartizione ancora da verificare ma che potrebbe dipendere da criteri di distribuzione dei carichi di lavoro tra diverse maestranze e/o differenti luoghi di produzione. ${ }^{167}$ Anche a proposito della omogeneità degli elementi dell'ambone gli archeologi della Stanford University sollevano qualche dubbio, ${ }^{168}$ ma credo che, in questo caso, le differenze di aspetto

38-39. Aggiungo solo, a titolo di ipotesi, che la lavorazione parziale delle basi poteva rispondere all'esigenza dei committenti di defalcare una voce dai preventivi di spesa e quindi contenere i costi per l'approvvigionamento dei marmi, demandando eventualmente la finitura del lavoro ad un intervento da eseguirsi una volta che i pezzi dai centri di produzione fossero giunti a destinazione.

${ }^{163}$ Pensabene 1986, 348, Russo 2006, 245 (vedi anche Russo 2010, 37) appaiono più propensi a ritenere incompleta la lavorazione dei capitelli di Marzamemi; su questo aspetto rinvio alle riflessioni di Claudia Barsanti supra nota 32.

${ }^{164}$ Russo 2006, 245; Russo 2010, 37-39.

${ }^{165}$ Leidwanger, Tusa 2016, 138. A favore di una chiara provenienza dei marmi bianchi dalle cave del Proconneso si pronuncia ora Leidwanger 2018, 344.

${ }^{166}$ Per l'applicazione di analisi incrociate per la caratterizzazione dei marmi bianchi vedi Attanasio, Brilli, Bruno 2008.

${ }^{167}$ Attanasio, Brilli, Rocchi 2008. Sul complesso delle due chiese di Latrun e sul suo ricco apparato decorativo in marmo vedi Widrig 1978; Michel 2011.

${ }^{168}$ Leidwanger, Tusa 2016, 138-139. 
dei pezzi vadano imputate non all'impiego di marmi diversi ma ad una maggiore o minore resistenza dei singoli elementi all'aggressione dell'ambiente marino. ${ }^{169}$

I nuovi dati scaturiti dallo scavo, tuttora in corso, hanno modificato la stima complessiva del carico avanzata a suo tempo da Kapitän, secondo cui la nave avrebbe trasportato l'arredo marmoreo e liturgico per una basilica di dimensioni davvero ragguardevoli, con due file di 14 colonne: al momento i capitelli rinvenuti sono 33-35, mentre il numero delle colonne, anche se incerto, appare essere inferiore e non congruente: si potrebbe pensare eventualmente a una destinazione dei capitelli eccedenti il numero delle colonne per un nartece, o un portico, dotato di sostegni già presenti a destinazione o da reperire in altro modo.

Altro elemento qualificante dello standard elevato dell'edificio è la presenza dell'ambone in verde antico, esclusivo per quel che ne sappiamo, solo di grandi fondazioni della capitale o di Tessalonica. ${ }^{170}$ Credo che per cercare di delineare l'identikit della committenza e per avanzare qualche ipotesi circa l'ubicazione dell' edificio cui i marmi erano destinati, non sia disutile anche riflettere sulle "assenze" nel carico: in esso non troviamo ad esempio colonne, capitelli e basi di dimensioni adatte ad una galleria sovrapposta alle navatelle, segno che la chiesa in questione non ne prevedeva la realizzazione. Non troviamo nemmeno pulvini, utilizzati invece nella chiesa occidentale di Latrun, edificio con il quale i marmi di Marzamemi vengono frequentemente accostati; i pulvini sono anche un marchio di fabbrica delle basiliche di Ravenna, città verso la quale gli archeologi della Stanford University sarebbero orientati a "destinare" il carico di Marzamemi, ${ }^{171}$ rispetto a questa ipotesi, ancora molto sommessamente avanzata, mi sento di dire che militino a sfavore il dato negativo dei pulvini, la scarsissima popolarità del capitello tipo VII che non trova alcuna attestazione nel ricco parterre della scultura in funzione architettonica della città, e la stessa difficoltà a trovare uno spazio idoneo ad un edificio di queste dimensioni e caratteristiche nel panorama architettonico e topografico di Ravenna, così come lo conosciamo tanto dalle emergenze monumentali quanto dalle testimonianze delle fonti scritte. ${ }^{172}$

Per concludere l'elenco delle "assenze", va sottolineato inoltre che nel carico, allo stato attuale, sembrerebbero non trovar posto né lastre per rivestimenti parietali e pavimentali, né cornici e rivestimenti per le arcate; ${ }^{173}$ potremmo spiegare questo fatto con una spedizione differenziata tra materiali strutturali inviati in prima battuta per necessità costruttive e elementi decorativi destinati ad un altro cargo, oppure con il fatto che la chiesa in questione prevedeva un altro tipo di decorazione, come pavimentazioni a mosaico e rivestimenti in

\footnotetext{
${ }^{169}$ Sulle caratteristiche del verde antico di Tessaglia, le località di estrazione e la sua diffusione in età romana e bizantina vedi Karagiorgou 2004; Lazzarini 2007, 223-244; Melfos 2008.

${ }^{170}$ In verde antico era ad esempio l'ambone del San Giovanni Battista all'Hebdomon, i cui due unici frammenti si trovano ora distribuiti tra il Museo Archeologico e l'Ayasofya Müzesi di Istanbul (cfr. Flaminio 2010, 73-75). A Tessalonica erano in analogo marmo ma di diversa fattura l'ambone di Santa Sofia (ora conservato al Museo Archeologico di Istanbul, cfr. Karagiorgou 2004, 197, 204, fig. 12) e quello di San Mena, tuttora in situ.

${ }^{171}$ Leidwanger, Pike, Donnelly c.s.

${ }^{172}$ Per la sua vastità è impossibile condensare in una nota la bibliografia sugli arredi marmorei degli edifici di V e VI secolo di Ravenna, mi limito pertanto a rinviare genericamente alle fondamentali opere che Friederich Wilhelm Deichmann ha dedicato ai monumenti paleocristiani della città. Per una valutazione dei flussi di manufatti marmorei inviati tra V e VI secolo verso la città vedi Harper 1997.

${ }^{173}$ Vedi però le più recenti informazioni sull'avanzamento delle indagini archeologiche in Leidwanger, Greene 2017; Leidwanger 2018, 347 e 350, fig. 14.
} 
stucco o ad intonaco dipinto. Mancano infine dati epigrafici, come monogrammi apposti su capitelli o lastre concernenti la committenza.

Nonostante tutte queste speculazioni, la ricerca della meta cui era diretta la nave naufragata lungo le coste della Sicilia orientale è ben lontana dal trovare una soluzione, né è possibile al momento delineare con un certo grado di precisione il profilo del committente che aveva richiesto questo lussuoso set di marmi. ${ }^{174}$ Resta solo da sottolineare l'importanza del relitto di Marzamemi nel più ampio quadro del commercio dei marmi in epoca paleobizantina - riflesso di un sofisticato sistema economico, politico e ideologico in cui lo splendore e la nobiltà di quel materiale giocava un ruolo niente affatto secondario - in attesa che nuove acquisizioni dagli scavi in corso e l'approfondimento delle conoscenze di altri relitti con carichi marmorei solo superficialmente noti, possano contribuire a definire meglio dinamiche e organizzazione dei processi produttivi. ${ }^{175}$

Vorrei concludere questo intervento con alcune riflessioni e nuove acquisizioni su un tema che mi è particolarmente caro e che ha a che vedere con le fasi conclusive della messa in opera dei manufatti marmorei e litici nell'ambito del cantiere. Alludo alla pratica della siglatura delle membrature marmoree, che ho avuto modo di studiare nell'ambito della ricerca diretta da Alessandra Guiglia e Claudia Barsanti e di cui hanno fatto parte anche Roberta Flaminio, Mauro della Valle e Asnu Bilban Yalçin e che ha dato esito, nel 2004, ad una monografia ${ }^{176}$ dedicata, in primo luogo, al catalogo dei plutei degli intercolumni e dei finestrati delle gallerie, ${ }^{177}$ con approfondimenti inoltre sui marmi reimpiegati, ${ }^{178}$ sui restauri ottocenteschi $^{179}$ e sulle sigle dei marmorari. ${ }^{180}$ In quell'occasione mi sono dedicato a quel vastissimo complesso di sigle ostentatamente apposte pressoché su tutte le membrature marmoree dell'edificio - che già nel passato avevano catturato la curiosità degli studiosi ${ }^{181}$ - ricono-

\footnotetext{
${ }^{174}$ Tra le possibili rotte della nave naufragata a Marzamemi quella che conduceva alle coste africane è vista in genere dagli studiosi come la più verosimile (cfr. ad esempio Kapitän 1980, 129: Harrison 1985; Sodini 1989, 167; contra, ma con argomentazioni non esplicite, Leone 2013, 197), senza trascurare però la possibilità di una destinazione siciliana (Deichmann 1976, 227; Castagnino Berlinghieri, Paribeni 2011, 71-72; Castagnino Berlinghieri, Paribeni 2015b, 398). Vorrei comunque sottolineare come la ricca documentazione dei flussi di arredi marmorei di VI secolo dagli opifici costantinopolitani verso Roma (Barsanti 2002; Guiglia Guidobaldi 2002) e Ravenna pres cinda dall'effettivo controllo politico militare esercitato dai bizantini in quelle realtà (Castagnino Berlinghieri, Paribeni 2015b, 397); anzi a Roma le testimonianze paiono essere più cospicue prima che dopo il 540, come se il conferimento di tali arredi squisitamente bizantini rientrasse in un piano di 'pacifica penetrazione' dei territori italiani da conquistare eventualmente in seguito manu militari; alla luce di queste considerazioni andrebbero dunque calibrate talune assertive definizioni (Ward Perkins 1951, 100, 103; Leone 2013, 195-202, anche a proposito del relitto di Marzamemi) secondo le quali i marmi prodotti negli opifici della capitale avrebbero potuto raggiungere località dell'Africa o della Sicilia soltanto in conseguenza della reconquista bizantina degli anni '40 del VI secolo.

${ }^{175}$ Negli ultimi anni altri relitti navali con carichi di marmi ascrivibili alla prima età bizantina sono stati individuati in diverse località del Mar di Marmara, dell'Egeo e del Mediterraneo orientale, ma le informazioni disponibili al momento sono troppo frammentarie e superficiali per poter azzardare una lettura di insieme di questi avvistamenti. Cfr. per il momento Pulak, Rogers 1994, 17; Günsenin 1998, 309; Dennert. Westphalen 2004; Castagnino Berlinghieri, Paribeni 2011, 66; Russell 2013b, 332, 334; Marano 2014, 423; Toma 2018, 166-167.

${ }^{176}$ Guiglia Guidobaldi, Barsanti 2004.

${ }^{177}$ Barsanti 2004; Guiglia Guidobaldi 2004; vedi anche per i soffitti di architrave al di sopra dei finestrati Yalçin 2004.

${ }^{178}$ Flaminio 2004.

${ }^{179}$ Della Valle 2004.

${ }^{180}$ Paribeni 2004.

${ }^{181}$ Rinvio al primo inventario generale delle sigle fatto da Antoniades 1907-1909, I, 97-103. Ricognizioni più specifiche furono condotte da Lawrence Butler per gli elementi che compongono le cornici inferiore e superiore prospettanti il naos
} 
scendovi in esse il senso di una orgogliosa affermazione dell'identità dei lapicidi e suggerendo come una loro sistematica mappatura potesse fornire uno strumento prezioso per individuare e comprendere dinamiche di cantiere e strategie operative messe in campo dai mechanikoi Antemio e Isidoro i quali, in soli cinque anni, dal 532 al 537, riuscirono nell'impresa di erigere dalle macerie della precedente, la Grande Chiesa voluta da Giustiniano. In questa sede vorrei invece soffermarmi su sigle che possono ricondursi ad altre finalità e che, in maniera meno vistosa delle precedenti, sono comunque attestate all'interno della Santa Sofia. ${ }^{182}$

Un primo caso, ancora tutto da comprendere ed inquadrare convincentemente, è suggerito dalla sigla monoletterale $\pi$, attestata quasi unicamente sui battiscopa del naos e del nartece, ${ }^{183}$ oltre che su poche cimase e lastre del finestrato ovest e, come ho avuto modo di verificare nei sopralluoghi compiuti nel 2013, sui montanti del secondo e terzo portale in pavonazzetto che dal nartece immettono nel naos, ove $\pi$ è in associazione con ZKO. Ad eccezione di due cimase, essa non compare mai da sola, ma sempre in sostegno di altre sigle di carattere certamente identitario (FIG. 60), che non si possono ricondurre ad un gruppo specifico. Si può verosimilmente interpretare la sigla in questione come un segno non identitario, ma da porre in relazione a procedure di messa in opera o di verifica e collaudo dei lavori eseguiti dalle maestranze. ${ }^{184}$

La riprova che nella Santa Sofia giustinianea le indicazioni per la messa in opera dei marmi erano fornite alle maestranze da una rete capillare di sigle alfabetiche ci è stata offerta nel 2013 da una fortunata occasione, verificatasi in coincidenza con la missione presso 1'Ayasofya Müzesi composta da Alessandra Guiglia, Claudia Barsanti, Silvia Pedone e da chi scrive: l'installazione di ponteggi a ridosso del colonnato nord del naos che, in quel momento, avevano raggiunto il livello dei capitelli e delle arcate, ci ha permesso di godere del raro privilegio di un contatto ravvicinato con porzioni del monumento fino ad allora esaminate soltanto da terra. In primo luogo abbiamo potuto riscontrare la presenza di un notevole numero di sigle apposte sull'abaco dei monumentali Kesselkapitelle (FIG. 61), il che ci ha consentito di convalidare il convincimento che anche i capitelli, pur nell'esiguo spazio lasciato dalla lavorazione a giorno, non fossero stati esenti dalla sistematica siglatura che interessa pressoché tutte le membrature marmoree della Santa Sofia; ${ }^{185}$ nel dettaglio il primo capitello a partire da ovest presenta le sigle BO e AY in legatura; il capitello a seguire la sigla ПYP, mentre nel quarto si contano, sui lati ispezionabili dell'abaco, ben sei sigle: IO, AY in legatura (riprodotta due volte), $Z \Omega, \Pi$ e, di dubbia lettura, ON; va rimarcato come l'ispezione dei monumentali capitelli sia assolutamente parziale, in quanto il lato opposto a quello verso il naos e lontano dai ponteggi è fatalmente inattingibile e quindi l'eventuale presenza di sigle su quelle porzioni di abaco non è verificabile.

\footnotetext{
(Butler 1990). Ulteriori fondamentali riflessioni in Deichmann 1976, 206-230. Sulle sigle dei marmorari di età paleobizantina vedi ora Marsili 2015.

${ }^{182}$ Una prima segnalazione di queste nuove acquisizioni nel novero delle sigle di marmorari esistenti a S Sofia in Paribeni 2017, 747-748; Paribeni in c.d.s.

${ }^{183} \mathrm{Su}$ questi elementi vedi in generale Russo 2011, 25-31.

${ }^{184}$ Per alcune considerazioni sulla possibile funzione di questa sigla vedi Paribeni 2013, 1473.

${ }^{185}$ Paribeni 2004, 655 e 679 nota 107 per la bibliografia precedente. Sui capitelli della Santa Sofia vedi ora Russo 2012 e - troppo tardi per poterne rendere conto diffusamente - Russo 2017 (2018), 58-113 per la sistematica ricognizione delle sigle apposte sui capitelli.
} 
Ancor più ricca di spunti è stata l'osservazione dettagliata delle trine marmoree poste a rivestimento delle arcate e dei pennacchi che, per effetto della lavorazione a giorno, si staccano elegantemente dal fondo, il quale ancora conserva cospicue tracce di una vivace colorazione in azzurro; il profilo delle arcate è segnato da due fregi delimitati da listelli - in quello inferiore un tralcio d'acanto concluso in chiave d'arco da un clipeo con croce monogrammatica; in quello superiore sequenza di foglie a cinque punte tangenti tra loro. Il rivestimento delle arcate è realizzato per segmenti - per la precisione un elemento in chiave d'arco, due coppie simmetriche lungo l'estradosso e altri due elementi sagomati ad U che fungono da imposta il cui coerente accostamento è determinato da minutissime sigle di assemblaggio apposte sul listello mediano in corrispondenza delle giunture; le letterine si corrispondono a coppie, secondo una cadenza che rispetta l'ordine alfabetico; non tutte le lettere si riescono a leggere, ma è possibile individuare il punto di partenza della numerazione nell' elemento di imposta in corrispondenza della prima colonna a partire da ovest, dove, incisa sul listello, si distingue chiaramente una A. Proseguendo verso est lettere uguali si individuano nel punto di contatto tra dodicesimo e tredicesimo segmento (coppia di $\mathrm{H}$ ), tra tredicesimo e quattordicesimo segmento (coppia di $\Theta$, FIG. 62), tra quattordicesimo e quindicesimo segmento (coppia di I). Ancora più avanti, tra ventitreesimo e ventiseiesimo segmento si ricostruisce agevolmente una successione di coppie di sigle: O-O; П-П FIG. 63); P-P.

Come ho già detto non tutte le sigle sono leggibili, anzi per alcuni elementi forse le sigle non erano nemmeno previste, dato che, ad esempio, mancano sistematicamente nei segmenti in chiave d'arco, probabilmente perché ritenute superflue in quanto la posizione in questo caso era obbligata; comunque sia, pur nell'esiguità del tratto che abbiamo potuto ispezionare, appare evidente come, ancora una volta, anche attraverso questi semplici accorgimenti, risalti la razionale impostazione del lavoro all'interno del cantiere giustinianeo, ai cui due architetti, Antemio e Isidoro, Procopio di Cesarea, nel suo de Aedificiis, ascriveva tra i sommi meriti, quello di aver saputo attentamente regolare i compiti delle diverse maestranze. ${ }^{186}$

A.P.

Claudia Barsanti

University of Rome Tor Vergata

Andrea Paribeni

University of Urbino "Carlo Bo"

andrea.paribeni@uniurb.it

Referenze fotografiche: ove non altrimenti indicato le figure da 1 a 52 sono di Claudia Barsanti; quelle da 52 a 63 di Andrea Paribeni.

\footnotetext{
${ }^{186}$ Paribeni 2004, 715-716.
} 


\section{BIBLIOGRAPHY}

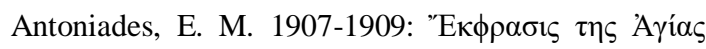

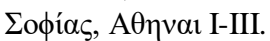

Arnaud, P. 2015: "Cities and Maritime Trade in the Roman Empire", in Chr. Schäfer (ed.), Connecting the Ancient World - Mediterranean Shipping, Maritime Networks and their Impact, Rahden (Pharos Studien zur griechisch-römischen Antike), 1-31.

Asgari, N. 1988: "The Stages of Workmanship of the Corinthian Capital in Proconnesus and Its Export Form", in N. Hertz, M. Waelkens (eds.), Classical Marble. Geochemistry, Technology, Trade, Dordrecht, 115-125.

Asgari, N. 1989: "Zwei Werkstücke für Konstantinopel aus den prokonnesischen Steinbrücken“, IstMitt 39, 49-63.

Asgari, N. 1990: “Objets de marbre finis, semi-finis et inachevés de Proconnèse", in M. Waelkens (ed.), Pierre éternelle du Nil au Rhin. Carrières et préfabrication, Bruxelles, 106-126.

Asgari, N. 1995: "The Proconnesian production of architectural elements in Late Antiquity, based on evidence from the marble quarries", in C. Mango, G. Dagron (eds.), Constantinople and its Hinterland, Aldershot, 263-288.

Asgari, N., Drew-Bear, Th. 2002: "The quarry inscriptions of Prokonnesos", in J.J. Herrmann Jr., N. Herz, R. Newman (eds.), ASMOSIA 5 Interdisciplinary Studies on Ancient Stone, London, 1-19.

Attanasio, D., Brilli, M., Bruno, M. 2008: "The properties and identification of marble from Proconnesos (Marmara Island, Turkey): a new database including isotopic, EPR and petrographic data", Archaeometry 50, 747-774.

Attanasio, D., Brilli, M., Rocchi, P. 2008: "The marbles of two early Christian churches at Latrun (Cyrenaica, Lybia)", in JASc 35, 1040-1048.

Baldini Lippolis, I. 2013: "Early byzantine churches in Creta and Cyprus between local identity and homologation", CCEC 43, 31-49.

Bardill, J. 2004: Brickstamps of Constantinople, Oxford. Bardill, J. 2008: "The Mason marks", in J. Crow, J. Bardill, R. Bayliss, The Water Supply of Byzantine Constantinople, London, 181-210.

Barsanti, C: 1982: "Scultura e architettura, ovvero alcuni aspetti del decoro scolpito negli edifici costantinopolitani del V-VI secolo", in Akten XVI. Internationales ByzantinistenKongress, Wien 1981 (JÖB, 34,2), Wien, 419-428.
Barsanti, C.: 1989 (1990), “L'esportazione di marmi dal Proconneso nelle regioni pontiche durante il IVVI secolo", RIASA III s., XII, 91-220.

Barsanti, C. 1995: "Il Foro di Teodosio I a Costantinopoli", in A. Iacobini, E.Zanini (eds.), Arte profana e arte sacra a Bisanzio, Roma, 9-50.

Barsanti, C. 1998: "I capitelli", in Sodini, Barsanti, Guiglia Guidobaldi 1998, 316-330.

Barsanti, C. 2002: "Capitelli di manifattura costantinopolitana a Roma", in Guidobaldi, Guiglia Guidobaldi (eds.) 2002, 1443-1478.

Barsanti, C. 2004: "I plutei degli intercolumni delle gallerie. Le cimase. Le transenne", in A. Guiglia Guidobaldi, C. Barsanti 2004, 315-529.

Barsanti, C. 2006: “I 'catini d'oro' di Padova; Spoglie costantinopolitane di VI secolo", in G. Trovabene (ed.), Florilegium artium. Scritti in memoria di Renato Polacco, Padova, 37-48.

Barsanti, C. 2013a: "Le cisterne bizantine di Istanbul: nuovi dati sulla scultura dal V al VII secolo. La cisterna n. 9 (la c.d. Cisterna della Scuola)", in Brandt et al., 2013, 1481-1496.

Barsanti, C. 2013b: "Una ricerca sulle sculture in opera nelle cisterne bizantine di Istanbul: la Ipek bodrum sarnıc1 (la cisterna n. 10)", in A. Rigo, A. Babuin, M. Trizio (eds.), Vie per Bisanzio, VIII Congresso Nazionale dell'Associazione Italiana di Studi Bizantini, Venezia, 25-28 novembre 2009, Bari, 477-508.

Barsanti, C. 2014: "Marmi costantinopolitani a Cipro", in M. Gianandrea, F. Gangemi, C. Costantini (eds.), Il potere dell'arte nel Medioevo. Studi in onore di Mario D’Onofrio, Roma, 195-206.

Barsanti, C. 2016: "Scultura dipinta a Bisanzio", in $\mathrm{Il}$ colore nel Medioevo. Arte, Simbolo, Tecnica tra materiali costitutivi e colori aggiunti mosaici, intarsi e plastica lapidea, Atti delle giornate di Studi (Lucca, 24-25-26 ottobre 2013), Lucca, 61-85.

Barsanti, C. 2017a: "Un nuovo capitello polilobato costantinopolitano", in C.L. Schiavi, S. Caldano, F. Gemelli (eds.), La lezione gentile. Scritti di storia dell'arte per Anna Maria Segagni Malacart, Milano, 47-58.

Barsanti, C. 2017b: "Capitelli corinzi del tipo cosiddetto 'a medaglione' con inserti decorativi”, in $\mathrm{P}$. Pensabene, M. Milella, F. Caprioli (eds.), Decor. Decorazione e architettura nel mondo romano, Atti del Convegno Internazionale, Roma, 21-24 maggio 2014, Roma, 391-400. 
Barsanti, C. in c.d.s: "A margine delle colonne-clava dell'arco del Foro di Teodosio I a Costantinopoli”, in Dialoghi con Bisanzio. Spazi di discussione, percorsi di ricerca, Atti dell'VIII Congresso Nazionale dell'Associazione Italiana di Studi Bizantini, Ravenna, 22-25 settembre 2015.

Barsanti, C., Flaminio, R., Guiglia Guidobaldi, A. 2015: Corpus della Scultura Medievale, VII, La diocesi di Roma. La III regione ecclesiastica, Spoleto.

Barsanti, C., Guiglia, A. 2010: The Sculptures of the Ayasofya Müzesi in Istanbul. A short guide, Istanbul.

Barsanti, C., Guiglia, A. 2018: "Spolia in Constantinople's Hagia Sophia from the Age of Justinian to the Ottoman Period: The Phenomenon of Multilayered Reuse", in I. Jevtić, S. Yalman (eds.), Spolia Reincarnated. Afterlives of Objects, Materials, and Spaces in Anatolia from Antiquity to the Ottoman Era, Istanbul, 97-123.

Barsanti, C., Guiglia Giudobaldi, A. 1996: "Premessa ad un catalogo della scultura della santa Sofia di Costantinopoli", in Bisanzio e l'Occidente. Arte, archeologia, storia. Studi in onore di Fernanda de' Maffei, Roma, 79-104.

Barsanti, C., Guiglia, A., Paribeni, A. 2010: "Le officine dell'imperatore: marmora byzantina", in $\mathrm{Me}$ dioevo: le officine, Atti del Convegno internazionale di studi, Parma, 22-27 settembre 2009, a cura di A.C. Quintavalle (I convegni di Parma, 12), Milano, 118151.

Barsanti, C., Paribeni, A. 2016: "La diffusione del marmo proconnesio nelle Marche in età classica e paleocristiana: il ruolo del porto di Ancona", Hortus Artium Medievalium 22, 200-214.

Barsanti, C., Pedone, S. in c.d.s.: "Scultura adriatica: alcune riflessioni sulla diffusione della tecnica en champlevé tra Grecia e Italia", in Bisanzio sulle due sponde del canale di Otranto, Atti dell'XI giornate dell'associazione italiana di studi bizantini (AISB), Lecce, 25-27 ottobre 2012.

Barsanti, C., Pilutti Namer, M. 2009: "Da Costantinopoli a Venezia: nuove spoglie della chiesa di S.

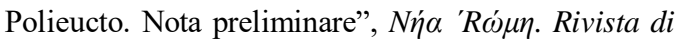
ricerche Bizantinistiche VI, 133-156.

Bassett, S. 2004: The Urban Image of Late Antique Constantinople, Cambridge.

Bauer, F.A. 1996: Stadt, Platz und Denkmal in der Spätantike: Untersuchungen zur Ausstattung des öffentlichen Raumes in den spätantiken Städten Rom, Konstantinopel und Ephesos, Mainz.

Bayezid II 2015: Bayezid II Turkish Bathhouse Culture Museum, Exhibition Catalogue, Istanbul 2015.

Beltrame, C., Lazzarini, L., Medaglia, S. 2012: "Underwater investigation on a marble cargo wreck at
Capo Bianco (Isola di Capo Rizzuto, Crotone, Italy)", IJNA 41, 3-16.

Beltrame, C., Vittorio, V. 2012: "Roman Ships carrying marble: were these vessels in some way special?", in Between Continents. Proceedings of the $12^{\text {th }}$ Symposium on Boat and Ship Archaeology, Istanbul, 142-148.

Berger, A. 1996: "Tauros e Sigma: Due piazze di Costantinopoli", in Bisanzio e l'Occidente. Arte, archeologia, storia. Studi in onore di Fernanda de' Maffei, Roma, 17-31.

Berger, A. 1997: "Regionen und Strassen im frühen Konstantinopel”, IstMitt 47, 349-414.

Berger, A. 2000: "Streets and public spaces in Constantinople", DOP 54, 161-174.

Bergmann, M., Heinzelmann, M. 2014: "Schedia Zollstation und Flusshafen Alexandrias am Kanopischen Nil", in S. Ladstätter, F. Pirson, Th. Schmidts (eds.), Häfen und Hafenstädte im östlichen Mittelmeerraum von der Antike bis in byzantinische Zeit. Neue Entdeckungen und aktuelle Forschungsansätze. Harbors and Harbor Cities in the Eastern Mediterranean from Antiquity to the Byzantine Period: Recent Discoveries and Current Approaches (Byzas 19), Istanbul, I, 101-112.

Betsch, W.E. 1979: The History, Production and Distribution of the Late Antique Capital in Constantinople (Ph. Diss. University of Pennsylvania, 1977), Ann Arbor.

Brandenburg, H. 2009: "Die Architektur und Bauskulptur von San Paolo fuori le mura. Baudekoration und Nutzung von Magazinmaterial im späteren 4.Jh", RM 115, 43-201.

Brandt, O. et al. 2013: Brandt, O., Cresci, S., López Quiroga, J., Pappalardo, C. (eds.), Episcopus, Civitas Territorium, Acta XV Congressus Internationalis Archaeologiae Christianae, Toleti, 8-12.9.2008 (Studi di Antichità Cristiana pubblicati a cura del Pontificio Istituto di Archeologia Cristiana, LXV), Città del Vaticano, I-III.

Brüx, R. 2008: Faltkapitelle. Untersuchungen zur Bauskulptur Konstantinopels, Langeneissbach.

Butler, L.E. 1985: "The nave cornices of Hagia Sophia: preliminary result of a study undertaken in 1983", AyasofyaMüzYl 10, 27-33.

Butler, L.E. 1990: The nave cornices of Hagia Sophia in Istanbul (Ph. Diss., Univ. of Pennsylvania 1989), Ann Arbor.

Butler, L.E. 1991: "Architectural Sculpture at H. Sergios and Bakchos and Hagia Sophia in Istanbul", in XVIII International Congress of Byzantine Studies. Summaries of Communications, Moscow, I, 194.

Butler, L.E. 1992: “Hagia Sophia's Nave Cornices as Elements of its Design and Structure", in R. Mark, 
A.S. Çakmak (eds.), The Haghia Sophia: from the Age of Justinian to the Present, Cambridge, 57-77.

Casson, S., Rice, D.T. et al. 1929: Second Preliminary Report upon the Excavations carried out in and near the Hippodrome of Constantinople in 1928, London.

Castagnino Berlinghieri, E.F. 2012: "The Pioneers of Underwater Archaeology", The Newsletter of Nautical Archaeology Society, Spring, 1-5.

Castagnino Berlinghieri, E.F. 2017: "Marzamemi II: Church Wreck", in P. Corby Finney (ed.), The Eerdmans Encyclopedia of Early Christian Art and Archaeology, W.B. Eerdmans Publishing Co., 117-120.

Castagnino Berlinghieri, E.F., Guzzardi, L. 2014: "“Nuovi" elementi architettonici della "basilica" a bordo della navis lapidaria di Marzamemi", in D. Leone, M. Turchiano, G. Volpe (eds.), Atti del III Convegno di Archeologia subacquea, Manfredonia 4-6 ottobre 2007, Bari, 49-56.

Castagnino Berlinghieri, E.F., Paribeni, A. 2011: "Byzantine merchant ships and marble trade: new data from the central Mediterranean", Skyllis 11, 64-75.

Castagnino Berlinghieri, E.F., Paribeni, A. 2015a: Marble production and marble trade along the Mediterranean coast in Early Byzantine Age (5th - 6th centuries): data from quarries, shipwrecks and monuments, in P.M. Militello, H. Oniz (eds.), SOMA 2011. Proceedings of the 15th Symposium on Mediterranean Archaeology, held at the University of Catania 3-5 March 2011, II (BAR Intern. Series 2695), Oxford, 1033-1041.

Castagnino Berlinghieri, E.F., Paribeni, A. 2015b: "Produzione e commercio del marmo lungo le rotte del Mediterraneo: evidenze dai depositi e dai relitti navali delle coste italiane", in Martorelli, Piras, Spanu 2015, 395-402.

Castagnino Berlinghieri, E.F., Paribeni, A. in c.d.s.: "Alcune colonne di un imprecisabile metallo. Avvistamenti e progetti di recupero del relitto di Marzamemi II nella prima metà del secolo scorso".

Clayton Fant, J.C. 2016: "A Milestone in the History of the Roman Trade in Stones", JRA 29, 701-710.

Croke, B. 2006: "Justinian, Theodora, and the Church of Saints Sergius and Bacchus”, DOP 60, 25-63.

Dark, K., Harris, A.L. 2008: "The Last Roman Forum: the Forum of Leo in Fifth-century Constantinople", GRBS 48, 57-69.

Deichmann, F.W. 1956: Studien zur ArchitekturKonstantinopels im 5. und 6. Jahrhundert nach Christus, Baden-Baden.

Deichmann, F.W. 1976: Ravenna. Hauptstadt des spätantiken Abendlandes (Kommentar, II,2), Wiesbaden.

della Valle, M. 2004: "I restauri ottocenteschi dei marmi e una prima affermazione del gusto neo- bizantino", in Guiglia Guidobaldi, Barsanti 2004, pp. 737-792.

Dennert, M. 2010: recensione a Brüx R. 2008, ByzZ 103, 199-202.

Dennert, M., Westphalen, S. 2004: "Säulen aus Konstantinopel - ein Schiffsfund im antiken Hafen von Amrit", DaM 14, 183-195.

Déroche, V. 1987: "L'acanthe de l'arc d'Hadrien et ses dérivés en Grèce propre”, $B C H$ 111, 425-453.

Detweiler, A. 1938: "The Triumphal Arch", in C.H. Kraeling (ed.), Gerasa, city of the Decapolis, New Haven, 73-83.

Di Bello, C. in c.d.s.: "I capitelli imposta del tipo cosiddetto a pannelli nell'ambito della produzione scultorea postgiustinianea", in D. Moreau (ed.), Archaeology of a World of Changes. Selected Papers on Late Roman and Early Byzantine Archaeology from the Non-Plenary Sessions of the 23rd International Congress of Byzantine Studies, Belgrade, 22-27 August 2016, Oxford.

Dodge, H., Ward-Perkins, B. (eds.) 1992: Marble in Antiquity, Collected Papers of J.B. Ward-Perkins (Archaeological Monographs of the British School at Rome, 6), Rome-London.

Ducrot, E. 1998: "Approccio metodologico per il riconoscimento della pietra antica: l'esempio della «Puddinga di Hereke»", in P. Pensabene (ed.), Marmi antichi II. Cave e tecnica di lavorazione provenienze e distribuzione (Studi Miscellanei 31), Roma, 79-83.

Duyuran, R. 1958: "Bayazit'da yapılan arkeolojik araştırma ve bazı müahedeler/Archaeological researches in Bayazit and some observations", IstanbAMüzYll 8, 71-73.

Ebersolt, J., Thiers A. 1913: Les églises de Constantinople, Paris.

Faedo, L. 1982: "Il complesso monumentale del Foro di Teodosio a Costantinopoli", CARB XXIX, 159-168.

Faedo, L. 1997: "Considerazioni sull'arco di Teodosio a Costantinopoli", CARB XLIII, 323-345.

Faedo, L. 1998: "Teodosio, Temistio e l'ideologia erculea nella Nea Rome. A proposito dell'arco del Forum Tauri”, RM 105, 315-328.

Feissel, D. 2000: "Les édifices de Justinien au témoignage de Procope et de l'épigraphie", AnTar 8, 81-104.

Firatl1, N. 1951: "Découverte de trois églises byzantines à Istanbul", CArch 5, 163-178.

Firatli, N. 1990: La sculpture byzantine figurée au Musée Archéologique d'Istanbul, catalogue revu et présenté par C. Metzger, A. Pralong et J.-P. Sodini, Paris.

Fischer, M. 1998: Marble Studies. Roma Palestine and the marble trade (Xenia, 40), Konstanz. 
Flaminio, R. 2004: "Gli elementi marmorei di reimpiego", in A. Guiglia Guidobaldi, C. Barsanti 2004, 533-648.

Flaminio, R. 2010: "Liturgical Furnishings", in C. Barsanti, A. Guiglia, The Sculptures of the Ayasofya Müzesi in Istanbul. A Short Guide, Istanbul, 69-77.

Gianfrotta, P.A. 2016: "Relitti con marmi (Naves lapidariae?): trasporti di stato, maestranze itineranti e coincidenze ostiensi”, $\mathrm{ACl} 77,341-359$.

Ginouvès, R. 1969: “Architecture”, in J. Des Gagniers, P. Devambez, L. Kahil, R. Ginouvès, Laodicée du Lycos: le nymphée campagnes 1961-1963, QuébecParis, 13-185.

Gough, M. 1985: Alahan. An Early Christian Monastery in Southern Turkey, Toronto.

Grabar, A. 1963: Sculptures byzantines de Constantinople (IVe-Xe siècle), Paris.

Greenhalgh, M. 2009: Marble Past, Monumental Present. Building with Antiquities in the Mediaeval Mediterranean (The Medieval Mediterranean. Peoples, Economies and Cultures, 400-1500, vol. 80), Leiden Boston.

Guidobaldi, F., Guiglia Guidobaldi, A. (eds.) 2002: Ecclesiae Urbis, Atti del Congresso Internazionale di Studi sulle Chiese di Roma (IV-X secolo), Roma 4-10 settembre 2000 (Studi di Antichità Cristiana pubblicati a cura del Pontificio Istituto di Archeologia Cristiana, LIX), Città del Vaticano, I-III.

Guidobaldi, F. 1994: "Cripta", in Enciclopedia dell'Arte Medievale V, Roma, 472-480.

Guiglia Guidobaldi, A. 1990: "I capitelli della basilica giustinianea della Theotokos, oggi di S. Caterina, sul Monte Sinai", in F. de Maffei, C. Barsanti, A. Guiglia Guidobaldi (eds.), Costantinopoli e l'arte delle province orientali, Roma, 265-342.

Guiglia Guidobaldi, A. 1995: "Reimpiego di marmi bizantini a Torcello", in A. Iacobini, E. Zanini (eds.), Arte profana e arte sacra a Bisanzio, Roma, 603-632.

Guiglia Guidobaldi, A. 1998: "Altri materiali scultorei in funzione architettonica e liturgica", in Sodini, Barsanti, Guiglia Guidobaldi 1998, 337-357.

Guiglia Guidobaldi, A. 2002: "La scultura di arredo liturgico nelle chiese di Roma: il momento bizantino", in F. Guidobaldi, A. Guiglia Guidobaldi (eds.) 2002, III, 1479-1524.

Guiglia Guidobaldi, A. 2004: "I finestrati delle gallerie. I plutei delle finestre. I soffitti non decorati degli architravi delle finestre della galleria occidentale. Il finestrone occidentale", in A. Guiglia Guidobaldi, C. Barsanti 2004, 89-311.

Guiglia Guidobaldi, A. 2007: “Appunti sulla scultura bizantina in Liguria”, in A. Calzona (ed.), Immagine e ideologia: studi in onore di Arturo Carlo Quintavalle, Milano, 34-46.
Guiglia Guidobaldi, A. 2010: "Da Costantinopoli a Genova e a Varese: insolito itinerario di una scultura bizantina del VI secolo", in A. Armati, M. Cerasoli, C. Luciani (eds.), Alle gentili arti ammaestra: studi in onore di Alkistis Proiou (Testi e Studi BizantinoNeoellenici XVIII), Roma, 97-113.

Guiglia Guidobaldi, A. 2011: "Le sculture bizantine di Trebisonda: appunti di viaggio", RIASA 3 s. 34=66, 131-150.

Guiglia Guidobaldi, A., Barsanti, C. 2004: Santa Sofia di Costantinopoli: l'arredo marmoreo della grande chiesa giustinianea (Studi di Antichità Cristiana pubblicati a cura del Pontificio Istituto di Archeologia Cristiana LX), Città del Vaticano.

Guiglia, A., Barsanti, C. 2008: "St. Sophia Museum Project 2006: The Marble Sculptures of the MiddleByzantine Period", 25. AST 2. Cilt (Kocaeli, $28 \mathrm{Ma}-$ y1s-1 Haziran 2007), Ankara, 117-132.

Guiglia, A, Barsanti, C. Flaminio, R. 2010: "Saint Sophia Museum Project 2009: The Collection of Byzantine Marble Slabs in The Ayasophia Müzesi”, 28. AST, 2. Cilt (Istanbul, 24-28 Mayıs 2010), Ankara, 361-376.

Guiglia, A., Barsanti, C., Paribeni, A., Yalçin, A.B. 2007: "Aya Sofya Projesi, 2005: Beyazılt Bölgesi Mermer Buluntuları”, 24, AST, 2. Cilt (Çannakale, 29 Mayıs-2 Haziran 2006), Ankara, 311-324.

Gül, M. 2009: The Emergence of Modern Istanbul. Transformation and Modernisation of a City, London-New York.

Günsenin, N. 1998: "Récentes découvertes sur l'île de Marmara (Proconnèse) à l'époque byzantine: épaves et lieux de chargement", Archaeonautica 14, 309-316.

Harper, J.G. 1997: "The provisioning of marble for the sixth-century churches of Ravenna: a reconstructive analysis", in R.L. Colella et al. (eds.), Pratum Romanum. Richard Krauthmeier zum 100. Geburtstag, Wiesbaden, 131-148.

Harrison, R.M. 1985: "The Building Materials of Churches in Cyrenaica”, in G. Barker, J. Lloyd, J. Reynolds (eds.), Cyrenaica in Antiquity (BAR Internat. Series 236), Oxford, 231-235.

Harrison, R.M. 1986: Excavations at Saraçane in Istanbul, I. The Excavations, Structures, Architectural Decoration, Small Finds, Coins, Bones, and Molluscs, Princeton.

Harrison, R.M. 1989: A Temple for Byzantium. The Discovery and Excavation of Anicia Juliana's Palace Church in Istanbul, Austin, Texas.

Harrison, R.M., Lawson, G.H. 1966: "The Mosaics in Front of the Villâyet Building in Istanbul", IstanbAMüzYll 13-14, 216-218. 
Herzfeld, E., Guyer, S. 1930: Meriamlik und Korikos. Zwei christliche Ruinenstätten des Rauhen Kilikien (Monumenta Asiae Minoris Antiqua, 2), Manchester.

Kahwagi-Janho, H. 2014: "Chapiteaux corinthiens d'époque romaine à Tyr”, Syria 91, 319-350.

Kapitän, G. 1980: "Elementi architettonici per una basilica dal relitto navale del VI secolo di Marzamemi (Siracusa)", CARB 27, 71-136.

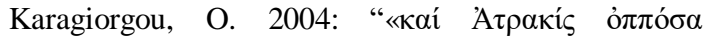

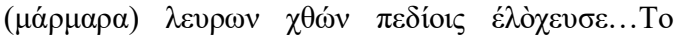

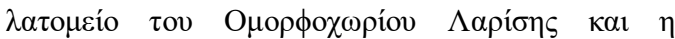

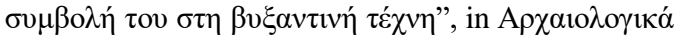

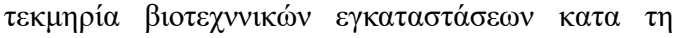

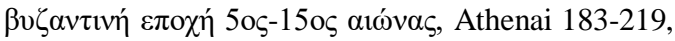
385-386.

Karagiorgou, O. 2015:“An early Byzantine stonemason and his workshop: New evidence from Amorium", in P. Petridis, B. Foskolou (eds), $\Delta \alpha \sigma \kappa \alpha \dot{\lambda} \alpha$.

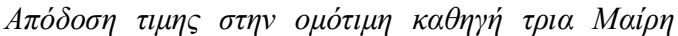

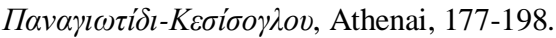

Kautzsch, R. 1936: Kapitellstudien. Beiträge zu einer Geschichte des spätantiken Kapitells im Osten von vierten bis ins siebente Jahrhundert, Berlin-Leipzig.

Kiilerich, B. 1993: Late fourth century classicism in the plastic arts: studies in the so-called Theodosian renaissance, Københaven.

Kiilerich, B. 2015: "The Barletta Colossus Revisited: The Methodological Challenges of an Enigmatic Statue", ActaArtHist XXVIII (N.S. 14), 55-72.

Kirımtayıf, S. 2001: Converted Byzantine Churches in Istanbul. Their Transformation into Mosques and Masjids, Istanbul.

Kleiss, W. 1974: "Bemerkungen zur Kirche Johannes des Täufers in Istanbul-Bakırköy (Hebdomon)", in Mansel'e Armağan. Mélanges Mansel, Türk Tarihi Kurumu Yayınları, S. 7, 60, Ankara, 207-219.

Kollwitz, J. 1941: Oströmische Plastik der theodosianischen Zeit, Berlin.

Kostenec, J. 2005-2006: "South façade of Sts. Sergius and Bacchus (Küçük Ayasofya Camii) in Istanbul", Boreas 28-29, 105-114.

Kramer, J. 1986: "Stilmerkmale korinthischer Kapitelle des ausgehenden 3. und des 4. Jahrhunderts n.Chr. in Kleinasien", in O. Feld, U. Peschlow (eds.), Studien zur spätantiken und byzantinischen Kunst, Friedrich Wilhelm Deichmann gewidmet, Bonn 109-126.

Kramer, J. 1997: Spätantike korintische Säulenkapitelle in Rom, Wiesbaden.

Kramer, J. 2006: Justinianische Kämpferkapitelle mit einem Dekor aus Paaren von Zweigen und die Nachfolgekapitelle im Veneto, Wiesbaden.

Lafontaine, J. 1959-1960: "Fouilles et découvertes byzantines à Istanbul de 1952 à 1960", Byzantion 2930, 339-396.
Lampinen, A. 2016: "A Helping Hand from the Divine on the Triumphalist Iconography of the Early Theodosians", Acta Byzantina Fennica 4, 9-38.

Lazzarini, L. 2007: Poikiloi lithoi, versiculores maculae: i marmi colorati della Grecia antica: storia, uso, diffusione, cave, geologia, caratterizzazione scientifica, archeometria, deterioramento (Marmora 1), PisaRoma.

Leidwanger, J. 2016: "I relitti di Marzamemi", in F. Agneto et al. (eds.), Mirabilia Maris. Tesori dai mari di Sicilia, Palermo, 185-187.

Leidwanger, J. 2018: "New investigations of the 6th c. A.D. "church wreck" at Marzamemi, Sicily", JRA 31, 339-356.

Leidwanger, J., Bruno, N. 2013: "Marzamemi II 'Church Wreck' excavation: 2013 field season”, Archaeologia Maritima Mediterranea 10, 191-198.

Leidwanger, J., Greene, E.S. 2017: “The Marzamemi Shipwreck Excavation: A Late Antique Church under the Sea", INA Quarterly 43, 3/4, 8-13.

Leidwanger, J., Pike, S.H., Donnelly, A. 2018: "Revisiting the Origin and Destination of the Late Antique Marzamemi 'Church Wreck' Cargo', in D. Matetić Poljak, K. Marasović (eds.), ASMOSIA XI. Interdisciplinary Studies on Ancient Stone, Proceedings of the Eleventh International Conference of ASMOSIA, Split, 18-22 May 2015, Split, 291-300.

Leidwanger, J., Tusa, S. 2015: "Marzamemi II 'Church Wreck’ excavation: 2014 field season”, Archaeologia Maritima Mediterranea 12, 103-115.

Leidwanger, J., Tusa, S. 2016: "Marzamemi II 'Church Wreck' excavation: 2015 field season”, Archaeologia Maritima Mediterranea 13, 129-143.

Leidwanger, J., Tusa, S. in c.d.s.: "Marzamemi II 'church wreck' excavation: 2017 field season," Archaeologia Maritima Mediterranea 15.

Leone, A. 2013: The End of the Pagan City. Religion, Economy, and Urbanism in Late Antique North Africa, Oxford.

Lethaby, W.R., Swainson, H. 1894: The Church of Sancta Sophia Constantinople. A Study of Byzantine Building, London, New York.

Mainstone, R.J. 1988: Hagia Sophia. Architecture, Structure and Liturgy of Justinian's Great Church, London.

Mango, C. 1972: The Art oft he Byzantine Empire 312 1453. Sources and Documents, Englewood Cliff.

Mango, C. 1977: "Storia dell'arte. Seminario 3"”, in La civiltà bizantina dal IV al IX secolo. Aspetti e problemi, Università degli Studi di Bari, Centro di Studi Bizantini, Corso di Studi I 1976, Bari, 316-321.

Mango, C. 1991: "Byzantine Epigraphy (IVth to Xth centuries)", in D. Harlfinger, G. Prato (ed.), Paleografia e codicologia greca. Atti del II colloquio in- 
ternazionale, Berlino-Wolfenbüttel 17-21 ottobre 1983, Alessandria, 115-146.

Mango, C., Hawkins, E.J.W. 1965: "The Apse Mosaic of St. Sophia at Istanbul. Report on Work carried out in 1964", DOP 19, 113-151.

Mango, C., Şevčenko, I. 1973: "Some churches and monasteries on the southern shore of the Sea of Marmara", DOP 27, 235-277.

Marano, J.A. 2014: "Le cave di marmo nella tarda antichità: aspetti organizzativi e produttivi", in J. Bonetto, S. Camporeale, A. Pizzo (eds.), Arqueologia de la construcción, IV Las canteras en el mundo antiguo: sistemas de explotación y procesos productivos, Actas del congreso de Padova, 22-24 de noviembre de 2012, Mérida, 413-427.

Marano, J.A. 2016: "The circulation of marble in the Adriatic Sea at the time of Justinian", in J. Herrin, J. Nelson (eds.), Ravenna: its role in early medieval change and exchange, London, 111-132.

Marsili, G. 2014a: “Per un'archeologia del lavoro in età tardoantica. Committenza, prassi organizzazione attraverso lo studio dei marchi dei marmorari sugli elementi di arredo architettonico e liturgico di marmo proconnesio, proposta di suddivisione tipologica", in Fonti per lo studio della cultura antica e medievale (DISCI), Bologna, 1-53.

Marsili, G. 2014b: "La committenza architettonica attraverso i marchi dei marmorari: il caso del Palazzo di Antioco a Costantinopoli" in P. Pensabene, C. Sfameni (eds.), La villa restaurata e $i$ nuovi studi sull'edilizia residenziale tardo antica, Atti del convegno internazionale del Centro Universitario si studi sull'Edilizia abitativa tardoantica nel Mediterraneo (CISEM), Piazza Armerina, 7-10 novembre 2012, 181-189.

Marsili, G. 2015: "Il cantiere e i marchi dei marmorari”, in I. Baldini, M. Livadiotti (eds.), Archeologia protobizantina a Kos. La città e il complesso episcopale, Bologna, 246-273.

Marsili, G. 2016: "La formazione dell'architetto in età protobizantina: il caso degli Isidori", in V. Neri, B. Girotti (eds.), La famiglia tardoantica: Società, diritto, religione, Milano, 239-255.

Martiny, G. 1947, in G. Brett, W.J. Macaulay, R. Stevenson, The Great Palace of the Byzantine Emperors. First Report, Oxford, 10-11.

Martorelli, A., Piras, A., Spanu, P.G. (eds.) 2015: Isole e terraferma nel primo cristianesimo. Identità locale ed interscambi culturali, religiosi e produttivi, Atti XI Congresso Nazionale di Archeologia Cristiana, Cagliari - Sant'Antioco, 23-27 settembre 2014 (Studi e Ricerche di Cultura Religiosa N.S. VIII), Cagliari.
Mathews, Th.F. 1971: The Early Churches of Constantinople. Architecture and Liturgy, London.

Mathews, Th.F. 1976: The Byzantine Churches of Istanbul. A Photographic Survey, London.

McKenzie, J. 2010: The Architecture of Alexandria and Egypt 300 BC - AD 700, New Haven-London.

Medaglia, S., Beltrame, C., Lazzarini, L. 2014: "La navis marmorum di età romana "Punta Scifo D" (Crotone). Risultati preliminari della prima campagna di indagini subacquee", $R d A 37,137-165$.

Melfos, V. 2008: "Green Thessalian stone: the byzantine quarries and the use of a unique architectural material from the Larisa area, Greece. Petrographic and geochemical characterization", OJA 27, 387-405.

Michel, V. 2011: "Nouvelles recherches en Cyrenaïque (Lybie): le site d'Érythron-Latrun", Les nouvelles de l'Archéologie 123, mars, 28-34.

Milella, M. 1996: "I propilei della santa Sofia teodosiana: continuità e trasformazione nella decorazione architettonica costantinopolitana del V secolo", in Bisanzio e l'Occidente: arte, archeologia, storia. Studi in onore di Fernanda de' Maffei, Roma, 61-78.

Müller Wiener, W. 1977: Bildlexikon zur Topographie Istanbuls, Tübingen.

Naumann, R. 1976: "Neue Beobachtungen am Theodosiusbogen und Forum Tauri in Istanbul", IstMitt 26, 117-141.

Naumann, R., Belting, H. 1966: Die Euphemia-Kirche am Hippodrom zu Istanbul und ihre Fresken, Berlin.

Niewöhner, Ph. 2007: Aizanoi, Dokimion und Anatolien. Stadt und Land, Siedlungs- und Steinmetzwesen von späteren 4. bis ins 6. Jahrhundert n. Chr., (Archäologische Forschungen 23), Wiesbaden.

Niewöhner, Ph. 2009: "Byzantinischen Gebälke im Archäologischen Museum Istanbul”, Araştırma Sonuçlari Toplantısı 3. Cilt (26-30 Mayıs 2008 Ankara), Ankara, 143-152.

Niewöhner, Ph. 2014: Production and Distribution of Docimian Marble in the Theodosian Age, in I. Jakobs (ed.), Production and Prosperity in the Theodosian Period, Leuven, 251-271.

Niewöhner, Ph. 2017 "The Decline and Afterlife of the Roman Entablature. The Collection of the Archaeological Museum Istanbul and other Epistyles and Cornices from Constantinople", IstMitt 67, 237-328.

Önlü, S. 2010: Yerebatan Sarnici'nin Taşıycı elemanlarını Analizi, Istanbul.

Ötüken, Y. 1996: Forschungen im nordwestlichen Kleinasien. Antike und byzantinische Denkmäler in der Provinz Bursa (IstMitt Beiheft 41), Tübingen.

Paribeni, A. 2004: "Le sigle dei marmorari e l'organizzazione del cantiere", in Guiglia Guidobaldi, Barsanti 2004, 649-734. 
Paribeni, A. 2013: "Modalità e prassi operative delle maestranze del Proconneso nei cantieri bizantini tra V e VI secolo", in Brandt et al. 2013, III, 1471-1480.

Paribeni, A. 2017: "Modalità di assemblaggio e messa in opera delle membrature architettoniche nei cantieri romani e bizantini (I-VI sec. d.C.): stato della questione e nuovi contributi", in Decor. Decorazione e architettura nel mondo romano, Atti del convegno, Roma 21-24 maggio 2014 (Thiasos monografie 9), Roma, 743-752.

Paribeni, A. in c.d.s.: "Riflessi dell'organizzazione del cantiere nel decoro scolpito della Santa Sofia di Costantinopoli”, in Dialoghi con Bisanzio. Spazi di discussione, percorsi di ricerca, Atti dell'VIII Congresso Nazionale dell'Associazione Italiana di Studi Bizantini, Ravenna, 22-25 settembre 2015.

Pasquini Vecchi, L. 1999; "La scultura di S. Polieucto: episodio saliente nel quadro della cultura artistica di Costantinopoli”, Bizantinistica. Rivista di Studi Bizantini e Slavi I, 109-144.

Pedone, S. 2013: "I monumenti di Costantinopoli della prima età bizantina nei disegni di Charles Texier (1802-1871)", in Brandt et al. 2013, III, 1591-1602.

Pedone, S. 2015: "Nuove considerazioni sulla scultura protobizantina delle isole tra importazioni e produzione locale: il caso della Sicilia”, in Martorelli, Piras, Spanu 2015, 377-394.

Pensabene, P. 1986: "La decorazione architettonica, l'impiego del marmo e l'importazione di manufatti orientali a Roma, in Italia e in Africa (II-VI sec. D.C.)", in A. Giardina (ed.), Società romana e impero tardoantico, III, Le merci, gli insegiamenti, Roma-Bari, 285-429.

Pensabene, P. 1993: Elementi architettonici di Alessandria e di altri siti egiziani (Repertorio dell'Egitto greco-romano $\mathrm{V}, 3$ ), Roma.

Pensabene, P. 1995: Le vie del marmo. I blocchi di cava di Roma e di Ostia. Il fenomeno del marmo nel mondo della Roma Antica, Roma.

Peschlow, U. 1986: "Eine wiedergewonnene byzantinische Ehrensäule in Istanbul", in O. Feld, U. Peschlow (eds.), Studien zur spätantiken und byzantinischen Kunst, 1. Friedrich Wilhelm Deichmann gewidmet, Bonn, 21-33.

Pralong, A. 1993: "Remarques sur les chapiteaux corinthiens tardifs en marbre de proconnèse", in L'acanthe dans la sculpture monumentale de l'Antiquité à la Renaissance, Actes du colloque tenu du $1^{\text {er }}$ au 5 octobre 1990 à la Sorbonne, Paris, 133-146.

Pralong, A. 2000: "La typologie de chapiteaux corinthiens tardifs en marbre de Proconnése et la production d'Alexandrie", $R A$ 1, 81-101.

Pulgher, D. 1878: Les anciennes églises byzantines de Constantinople, Vienne.
Russell, B. 2013a: The Economics of the Roman Stone Trade, Oxford.

Russell, B. 2013b: "Roman and Late-Antique Shipwrecks with Stone Cargoes: a New Inventory", JRA 26, 331-361.

Russo, E. 1999: "La cultura a Efeso in età paleocristiana e bizantina. Primi lineamenti", in Efeso Paleocristiana e Bizantina - Frühchristliches und byzantinisches Ephesos. Referate des vom 22. bis 24. Februar 1996 im Historischen Institut beim Österreichischen Kulturinstitut in Rom durchgeführten internationalen Kongresses aus Anlass des 100-jährigen Jubiläums der österreichischen Ausgrabungen in Ephesos, hrsg. von R. Pillinger, O. Kresten, F. Krinzinger, E. Russo (Österreichische Akademie der Wissenschaften Phil.hist. Kl. Denkschriften, 282. Band), Wien, 26-53.

Russo, E. 2004: "La scultura di S. Polieucto e la presenza della Persia nella cultura artistica di Costantinopoli nel VI secolo", in La Persia e Bisanzio, Atti del Convegno internazionale, Roma 14-18 ottobre 2002, Roma, 737-826.

Russo, E. 2006a, "La presenza degli artefici grecocostantinopolitani a Roma nel VI secolo", JÖAI 75, 243-297.

Russo, E. 2006b (2007): "La vera origine del capitello a cesto polilobato", Bizantinistica. Rivista di Studi Bizantini e Slavi II s. VIII, 61-84.

Russo, E. 2007a (2008): "La decorazione scultorea della S. Sofia di Costantinopoli", Bizantinistica. Rivista di Studi Bizantini e Slavi II s., IX, 1-14.

Russo, E. 2007b (2008): "Ancora il pulvino sopra il capitello a cesto", Bizantinistica. Rivista di Studi Bizantini e Slavi, II s., IX, 15-40.

Russo, E. 2008: "Costantinopoli. Architettura e scultura nei primi secoli", in Bisanzio, Costantinopoli, Istanbul, Milano, 45-62.

Russo, E. 2009a: "The sculptural decoration of the Theodosian Church of St. Sophia", 26. AST, 1. Cilt (Ankara, 26-30 Mayis 2008), Ankara, 155-166.

Russo, E. 2009b (2010): "Per la datazione della porta Aurea di Costantinopoli e dei suoi capitelli", Bizantinistica. Rivista di Studi Bizantini e Slavi II s., XI, 22-30.

Russo, E. 2010a: "Evidence from the Theodosian Saint Sophia", in C. Barsanti, A. Guiglia, The Sculptures of the Ayasophia Müzesi in Istanbul. A short guide, Istanbul, 19-34.

Russo, E. 2010b (2011): "La circolazione degli artefici e del marmo nel VI secolo", Bizantinistica. Rivista di Studi Bizantini e Slavi II s., XII, 31-55.

Russo, E. 2010: "La circolazione degli artefici e del marmo nel VI secolo", Bizantinistica Rivista di Studi Bizantini e Slavi II s., XII, 31-55. 
Russo, E. 2011: Le decorazioni di Isidoro il Giovane per S. Sofia di Costantinopoli, Roma.

Russo, E. 2012: "Introduzione ai capitelli di S. Sofia di Costantinopoli”, RIASA 67, III s., XXXV (2015), 95172.

Russo, E. 2015: "Il lapidario di Ayasofya a Istanbul. Le sculture architettoniche della chiesa di S. Sofia Teodosiana", in R. Martorelli (ed.), Itinerando. Senza confini dalla preistoria ad oggi. Studi in ricordo di Roberto Coroneo, I,1 Perugia, 301-324.

Russo, E. 2017 (2018): "Sulla lavorazione dei capitelli di S. Sofia di Costantinopoli", Bizantinistica Rivista di Studi Bizantini e Slavi II s., XVIII, 45-113.

Russo, E., Büyükkolanci, M. 2010: Sculture della prima basilica di San Giovanni a Efeso, (Quaderni della rivista di Bizantinistica 15), Spoleto.

Salzenberg, W. 1854: Alt-christliche Baudenkmale von Constantinopel vom V. bis XII, Jahrhundert, Berlin.

Sande, S. 1981: "Some new fragments from the Column of Theodosius", ActaArchHist. Series altera in $8^{\circ} 1,1-78$.

Schneider, A.M. 1941: Die Grabung im Westhof der Sophienkirche zu Istanbul, Berlin.

Sicily and the Sea 2015: Sicily and the Sea, cat. mostra Amsterdam, Allard Pierson Museum, 09.10.2014 17.04.2016, eds. D. Burgersdijk, R. Cali, J. Kerder, A. Sofroniew, S. Tusa, R. van Beek, Amsterdam.

Şimșek, C. 2007: Laodikeia (Laodikeia ad Lycum), Istanbul.

Sodini, J.-P. 1977: "Remarques sur la sculpture architecturale d'Attique, Béothie et du Péloponnèse à l'époque paléochrétienne", $B C H$ 101, 423-450.

Sodini, J.-P. 1989: "Le commerce des marbres à l'époque protobyzantine", in Hommes et richesses dans l'Empire byzantin Tome IV $V^{e}$-VII siècle (Réalités byzantines), Paris, 163-186.

Sodini, J.-P. 2000: "Le commerce des marbres dans la Méditerranée (IV $\left.{ }^{\mathrm{e}}-\mathrm{VII}{ }^{\mathrm{e}} \mathrm{s}.\right)$ ", in V Reunió d'arqueologia cristiana hispànica, Cartagena, 16-19 d'abril de 1998, Barcelona (Monografies de la Secció Històrico-Arqueològica, VII), 423-448.

Sodini, J.-P., Barsanti, C., Guiglia Guidobaldi, A. 1998: "La sculpture architecturale en marbre au $\mathrm{VI}^{\mathrm{e}}$ siècle à Constantinople et dans les regions sous influence constantinopolitaine", in Acta XIII Congressus Internationalis Archaeologiae Christianae, SplitPoreč 25.9-1.10.1994 (Studi di Antichità Cristiana pubblicati a cura del Pontificio Istituto di Archeologia Cristiana, LIV), Città del Vaticano, 301-376.

Sodini, J.-P., Kolokotsas, K. 1984: Aliki II: la basilique double (Etudes Thasiennes, $\mathrm{X}$ ), Paris.

Strube, Ch. 1984: Polyeuktoskirche und Hagia Sophia. Umbildung und Auflösung antiker Formen, Entstehen des Kämferkapitells (Bayerische Akademie der
Wissenschaften, Phil.-hist. Klasse, Abh. N.F. 92), München.

Swainson, H. 1895: "Monograms on the capitals of S. Sergius at Constantinople", Byzantinische Zeitschrift, 4, 106-108.

Swift, E.H. 1940: Hagia Sophia, New York.

Taddei, A. 2017: Hagia Sophia before Hagia Sophia. A study of the Great Church of Constantinople from its origins to the Nika Revolt of 532, Roma.

Toma, N. 2018: "Standardization and mass customization of architectural components: new perspectives on the Imperial marble construction industry", JRA 31, 161-191.

Türkmen, M.: Pamphylia ve Kilikia'da Severuslar Dönomim Mimar mezemesi, Doktora Tezi Istanbul Üniversitesi -Sosyal Bilimler Enstitüsü Arkeoloji Ana Bilim Dal, Klasik Arkeoloji Bilim Dalı.

Van Millingen. A. 1912: Byzantine churches in Constantinople: their history and architecture, London Verzone, P. 1956: "Il Tetrapilo aureo. Contributo alla topografia dell'antica Costantinopoli", MonAnt Serie miscellanea 43, 126-203.

Vignoli, A. 1978: "Note sull'architettura delle chiese dei Santi Sergio e Bacco a Costantinopoli. Ipotesi per la ricostruzione della struttura dell'avancorpo scomparso", StClOr XXVIII, 69-86.

Ward Perkins, J.B. 1951: "Tripolitania and the Marble Trade", JRS 41, 89-104.

Ward Perkins, J.B. 1980: "Nicomedia and the marble trade", PBSR XLVIII, 23-69.

Westall, R. 2011: "Constantius II and the Great Church

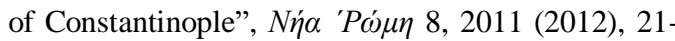
50 .

Wilkinson, J. 1987: Column capitals in al-Haram alSharif (from 138 A.D. to 1118 A.D.), Jerusalem.

Yalçin, A.B. 2004: "I soffitti decorati degli architravi delle finestre", in Guiglia Guidobaldi, Barsanti 2004, 235-289.

Yaylalı Yıldız, B. 2008: Stratified Ground of the City: Transformation of Istanbul Divanyolu in the Modern Period, Thesis of Middle East Technical University.

Yonkaci-Arslan, P. 2015: Christianizing the Skyline: Appropriation of the Pagan Honorary Column in Early Constantinople, UCLA, Architecture and Urban Design.

Yoncaci-Arslan, P. 2016: “Toward a New Honorific Column: The Column of Constantine in Early Byzantine Urban Landscape", Metu Jfa 4, 121-145.

Zollt, Th. 1994: Kapitellplastik Konstantinopels vom 4. bis 6. Jahrhundert n. Ch., Bonn. 


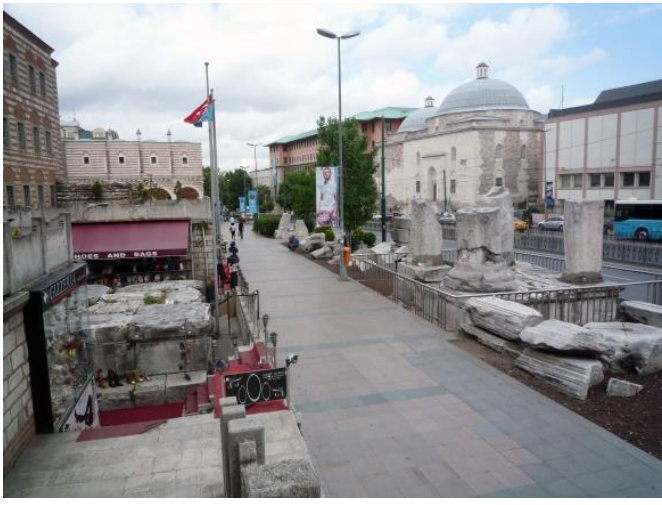

FIG. 1 - Istanbul, Ordu Caddesi: i basamenti dell'arco del Foro di Teodosio I.

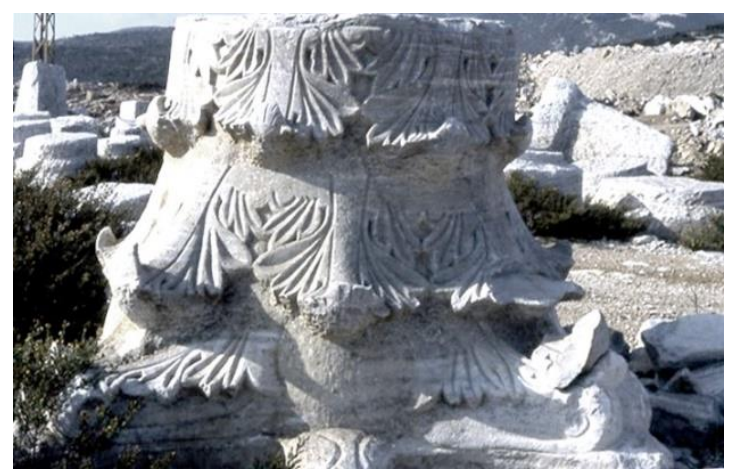

FIg. 3 - Marmara Adası (isola di Proconneso), area della cava di Silinte: capitello corinzio.

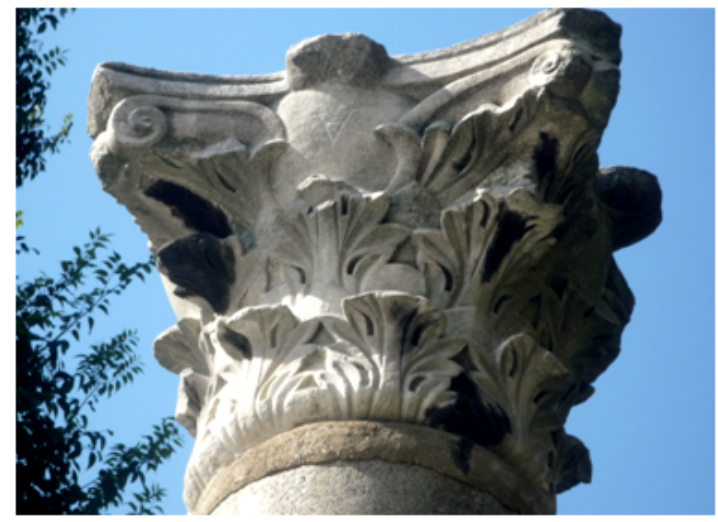

FIG. 5 - Istanbul, Ayasofya Müzesi: capitello corinzio attribuito al portico del prospetto teodosiano (inv. 134).

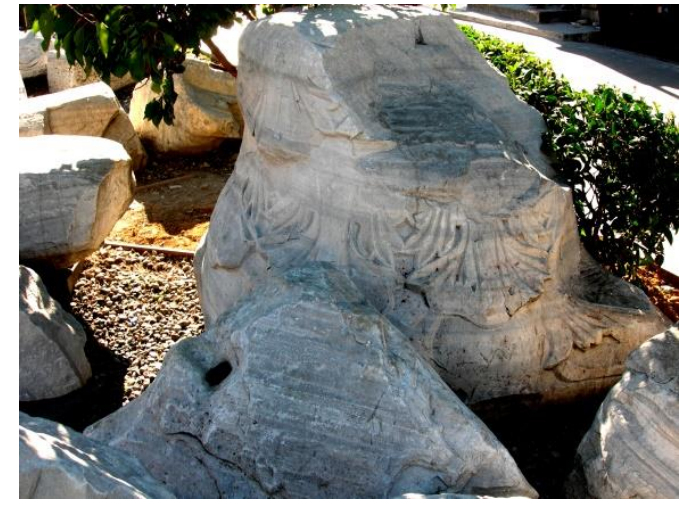

FIG. 2 - Istanbul, Ordu Caddesi: capitello corinzio A dell'arco del Foro di Teodosio I.

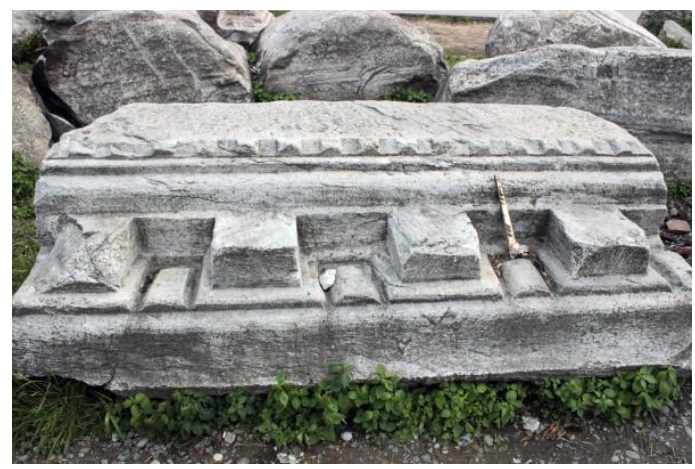

FIG. 4 - Istanbul, Ordu Caddesi: frammento di cornice a modiglioni 'non finita'.

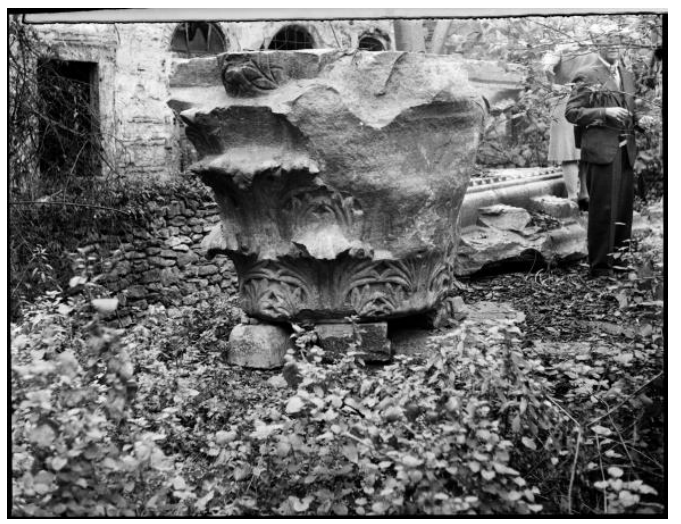

FIG. 6 - Istanbul, Simkeşhan: capitello corinzio B dell'arco del Foro di Teodosio I (foto N. Artamonoff 1945, DO CFA.NA 0341). 

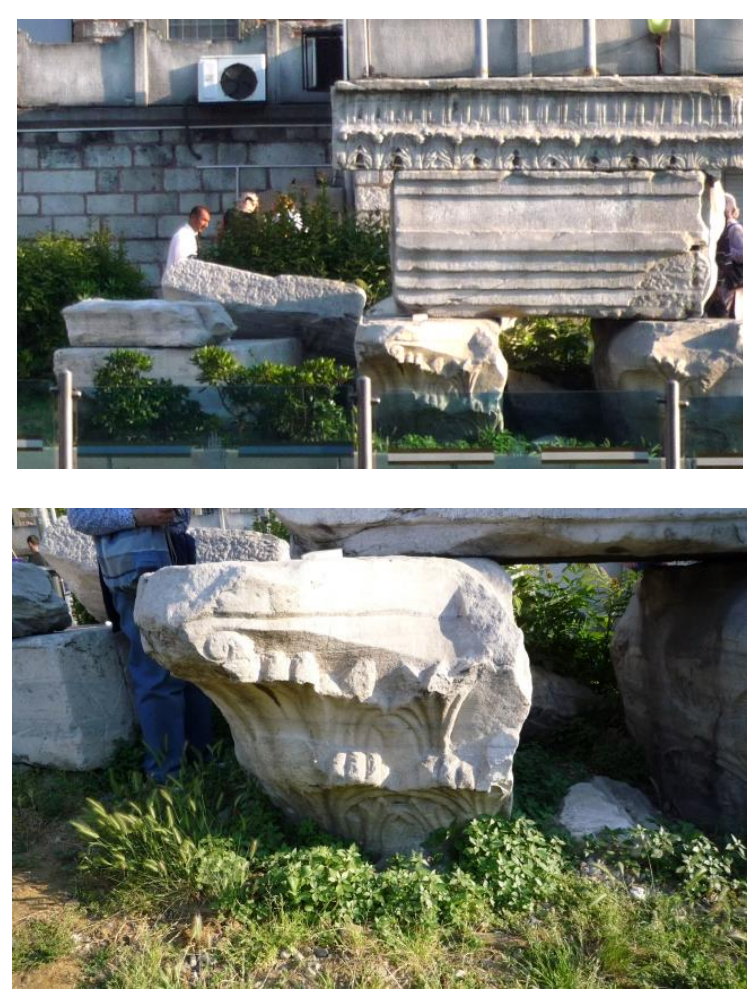

FIG. 8a - Istanbul, Ordu Caddesi: capitello corinzio B dell'arco del Foro di Teodosio I.

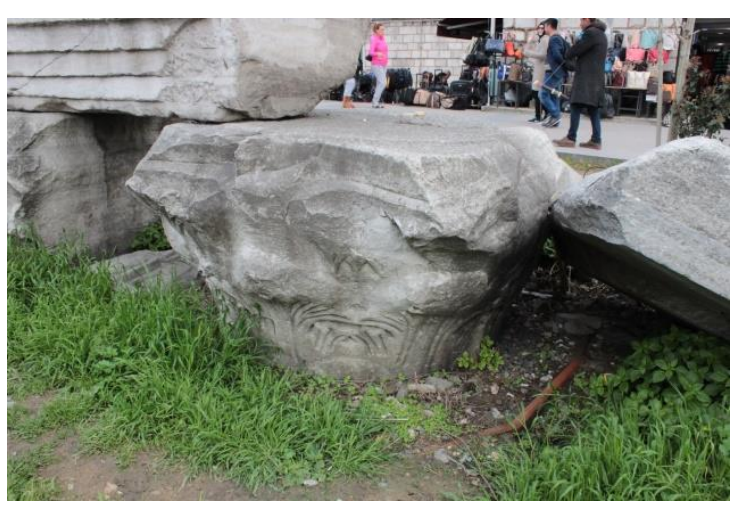

Fig. 9a - Istanbul, Ordu Caddesi: capitello corinzio C dell'arco del Foro di Teodosio I (foto Betsch 1970).
FIG. 7 - Istanbul, Ordu Caddesi: cornici e i capitelli B e C dell'arco del Foro di Teodosio I.

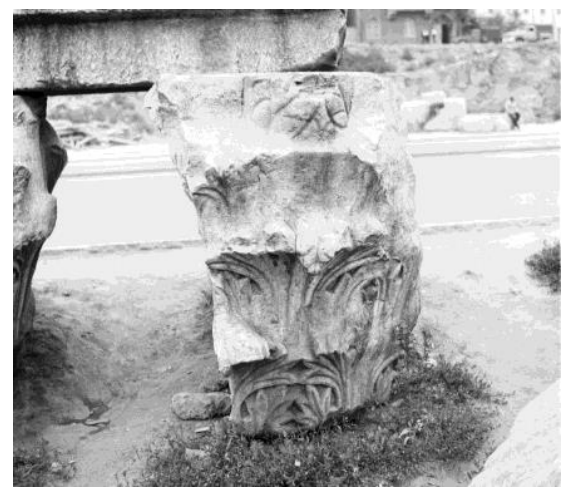

FIG. 8b - Istanbul, Ordu Caddesi: capitello corinzio B dell'arco del Foro di Teodosio I.

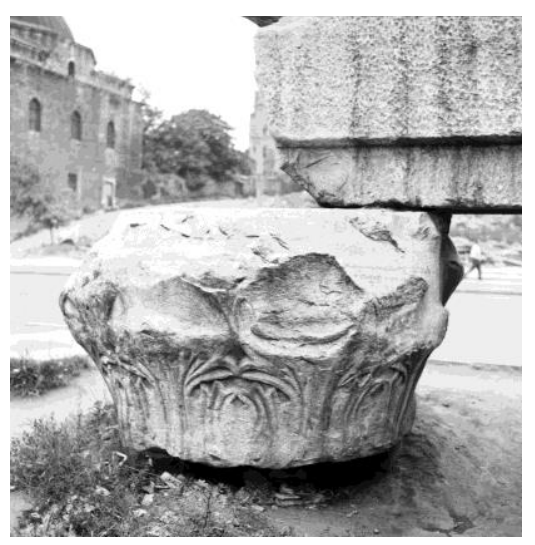

FIG. 9b - Istanbul, Ordu Caddesi: capitello corinzio C dell'arco del Foro di Teodosio I. 


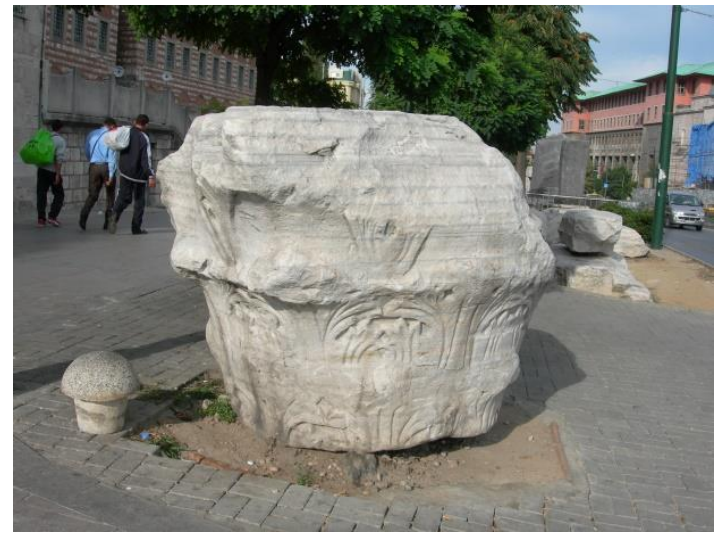

FIG. 10 - Istanbul, Ordu Caddesi: capitello corinzio D dell'arco del Foro di Teodosio I.

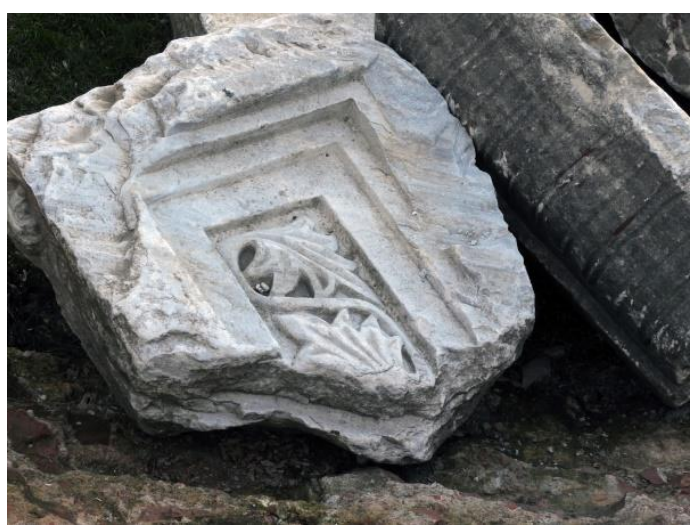

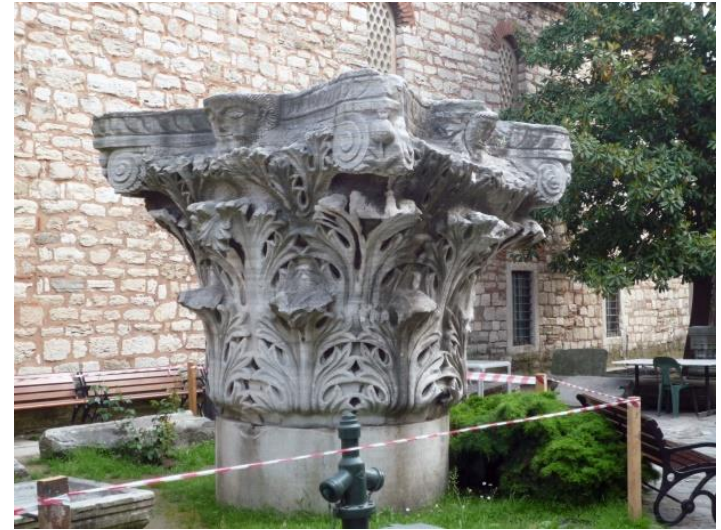

FIG. 11 - Istanbul, Topkapı Sarayı, cortile della Biblioteca: capitello corinzio della colonna onoraria di Leone I.

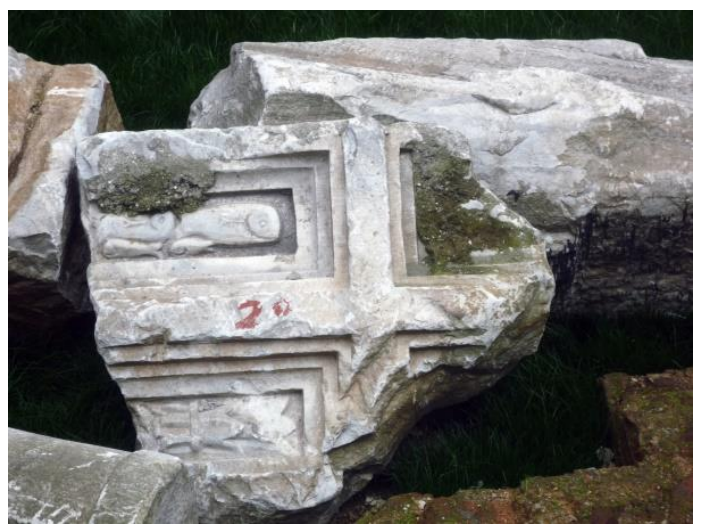

FIG. 12a b - Istanbul, Ordu Caddesi: frammenti di soffitto dell'arco del Foro di Teodosio I.

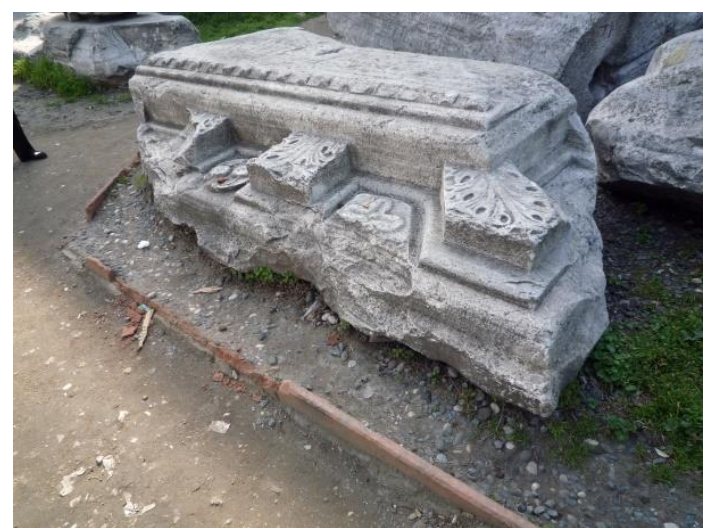

FIG. 13 - Istanbul, Ordu Caddesi: frammento della cornice di coronamento dell'arco del Foro di Teodosio I.

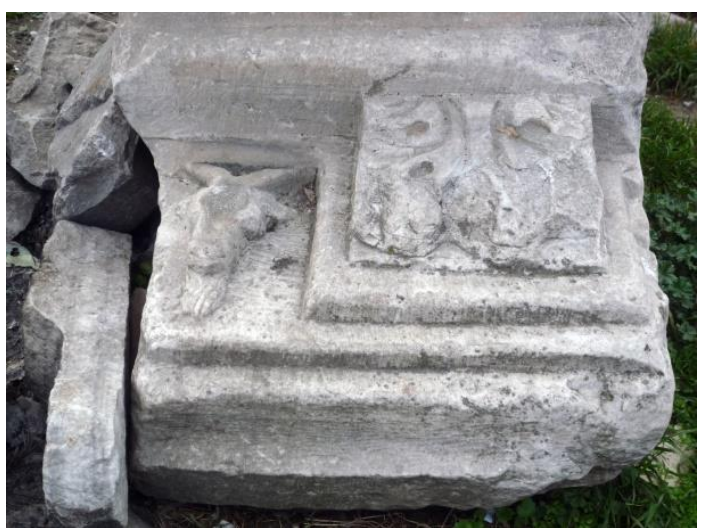

FIG. 14 - Istanbul, Ordu Caddesi: frammento della cornice di coronamento dell' arco del Foro di Teodosio I. 

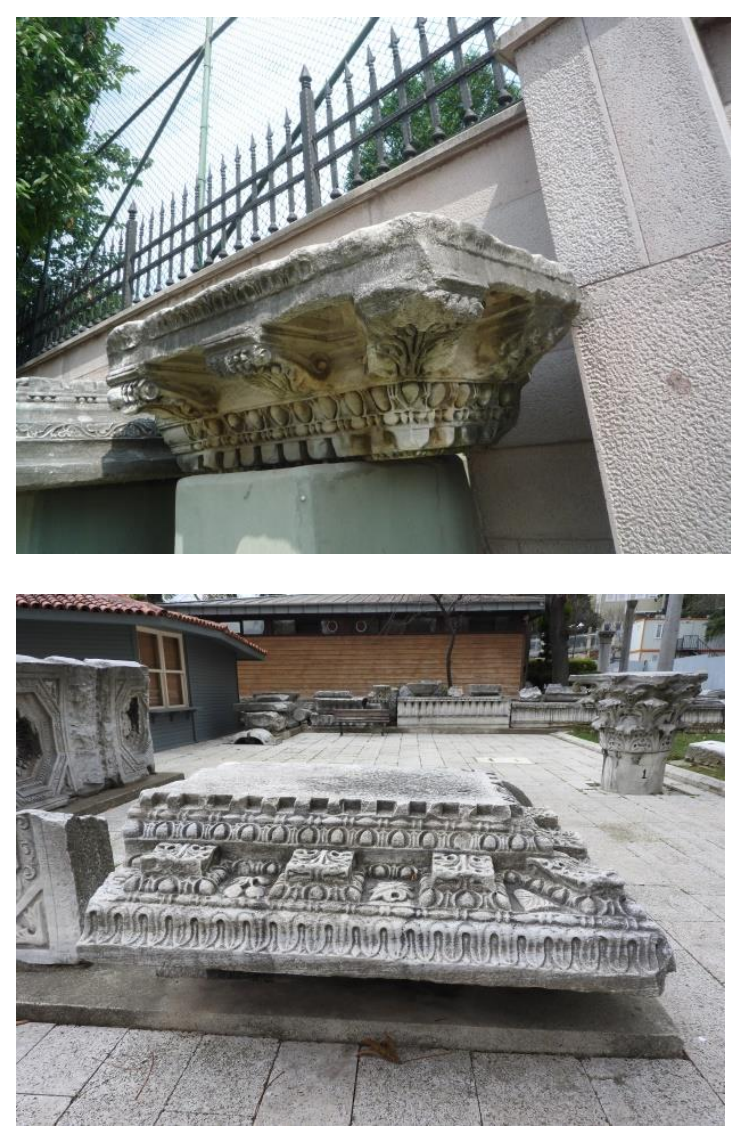

FIG. 16a - Istanbul, Ayasofya Müzesi: frammento angolare della cornice di coronamento del prospetto teodosiano.

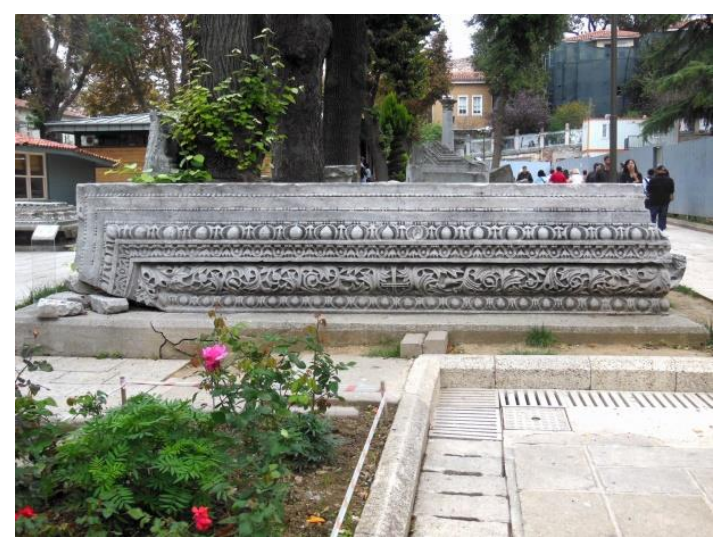

FIG. 17 - Istanbul, Ayasofya Müzesi: cornice del fastigio del prospetto teodosiano.
FIG. 15 - Izmit-Kocaeli, Museo Archeologico: frammento di cornice di coronamento.

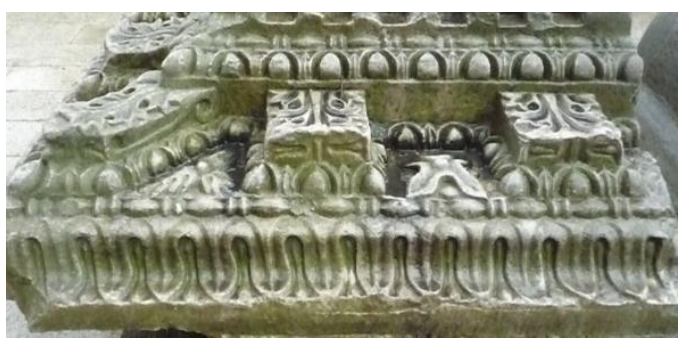

FIG. 16b - Istanbul, Ayasofya Müzesi: frammento angolare della cornice di coronamento del prospetto teodosiano (particolare).

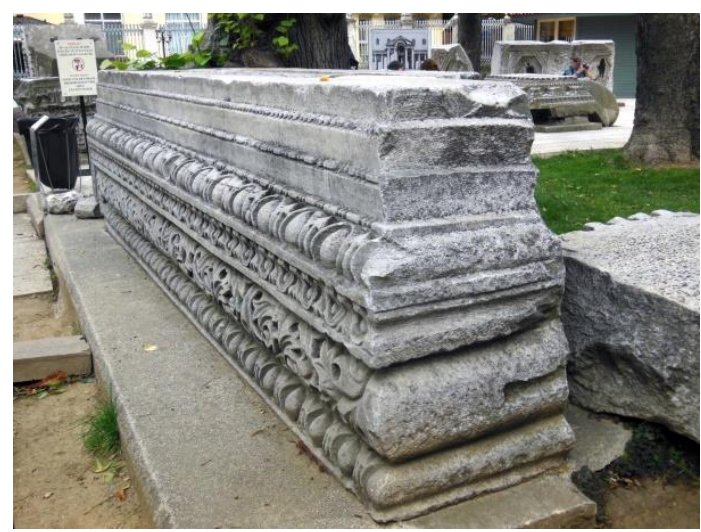

FIG. 18 - Istanbul, Ayasofya Müzesi: cornice del fastigio del prospetto teodosiano, particolare. 


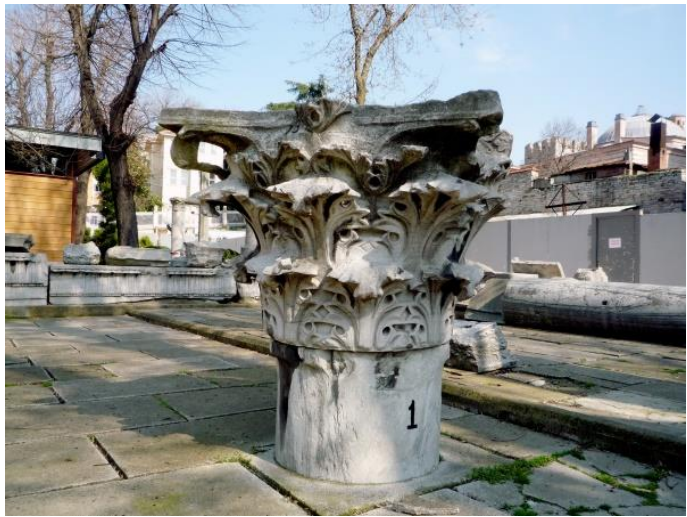

FIG. 19 - Istanbul, Ayasofya Müzesi: capitello corinzio (inv. 1) del fastigio del prospetto teodosiano.

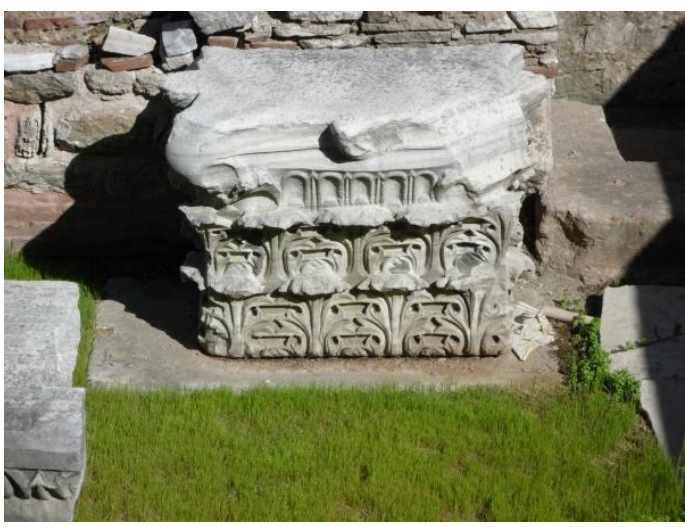

FIG. 21 - Istanbul, Ayasofya Müzesi: capitello corinzio di pilastro (inv. 83) del fastigio del prospetto teodosiano.

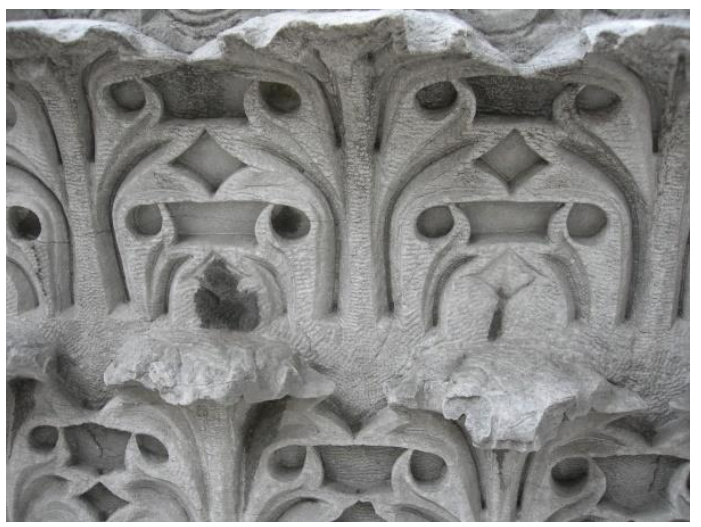

FIG. 23 - Istanbul, Ayasofya Müzesi: capitello corinzio di pilastro (inv. 19) del fastigio del prospetto teodosiano, particolare.

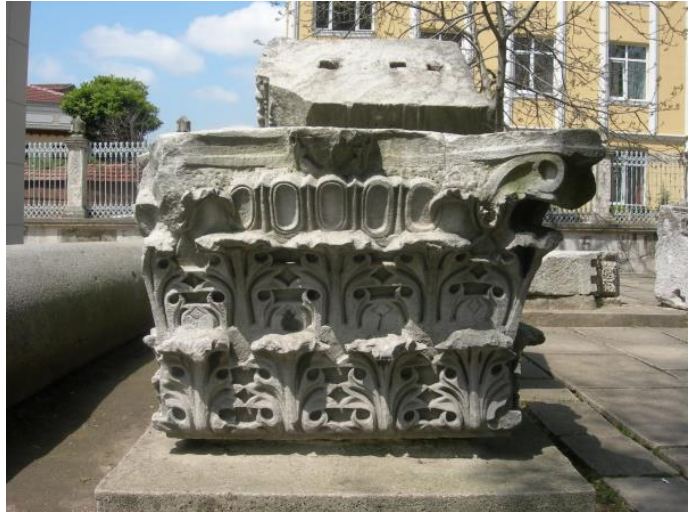

FIG. 20 - Istanbul, Ayasofya Müzesi: capitello corinzio di pilastro (inv. 19) del fastigio del prospetto teodosiano.

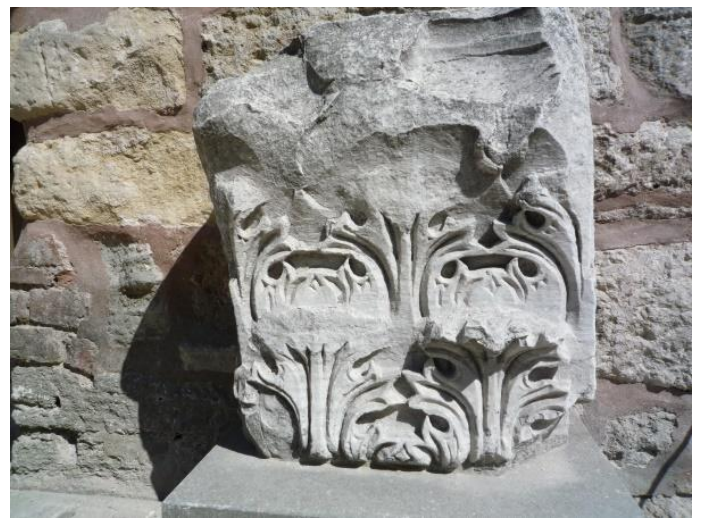

FIG. 22 - Istanbul, Ayasofya Müzesi: capitello corinzio di pilastro frammentario del fastigio del prospetto teodosiano.

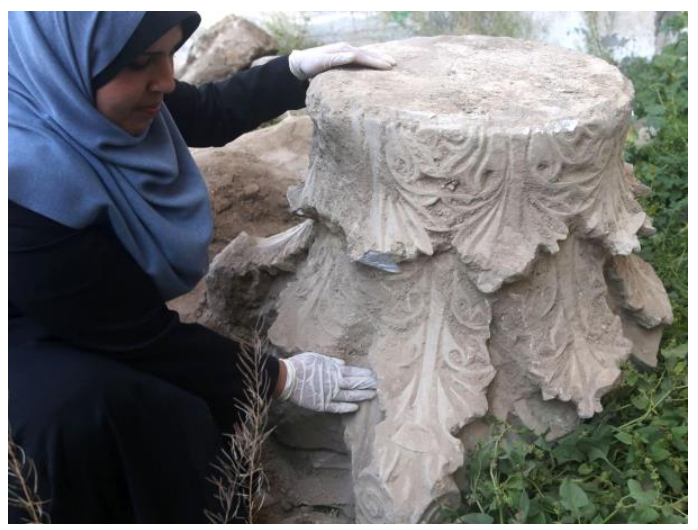

FIG. 24 - Gaza, elementi scultorei nel cantiere di un Supermercato (aprile 2016). 


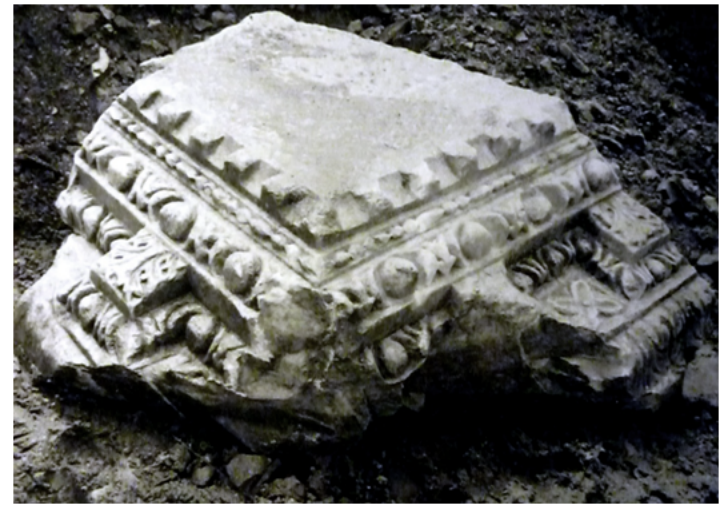

FIG. 25 - Istanbul, Museo Archeologico: frammento di cornice dalla chiesa di San Giovanni Battista all'Hebdomon (Neg. DAI Istanbul R317).

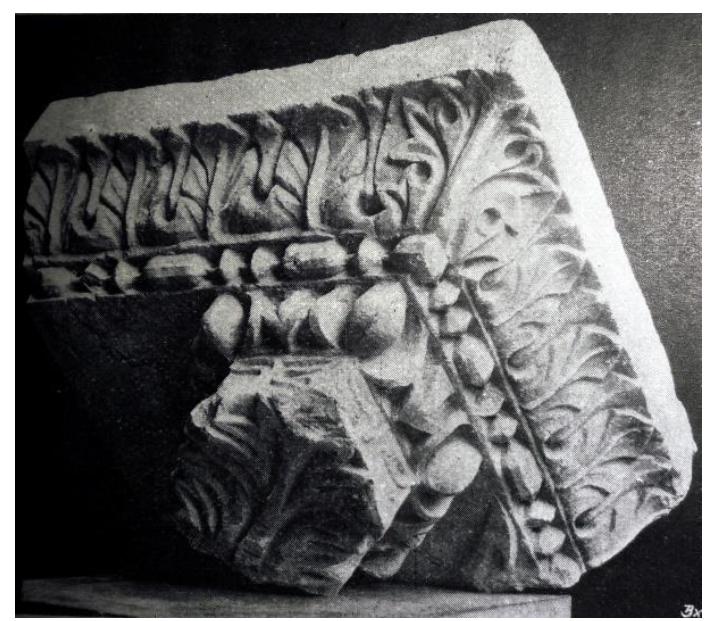

FIG. 27 - Berlino, Staatlichemuseen: frammento di cornice (da Wulff, Volbach 1923).

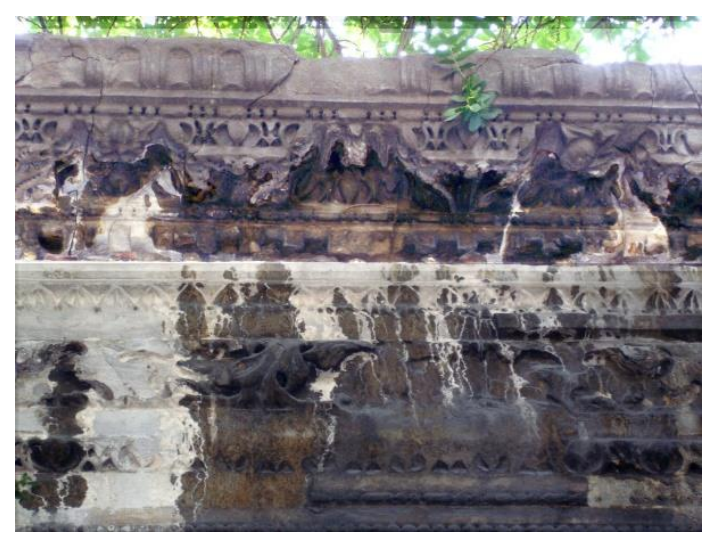

FIG. 29 - Istanbul, Basilica di San Giovanni di Studio (Imrahor Camii): trabeazione del nartece.

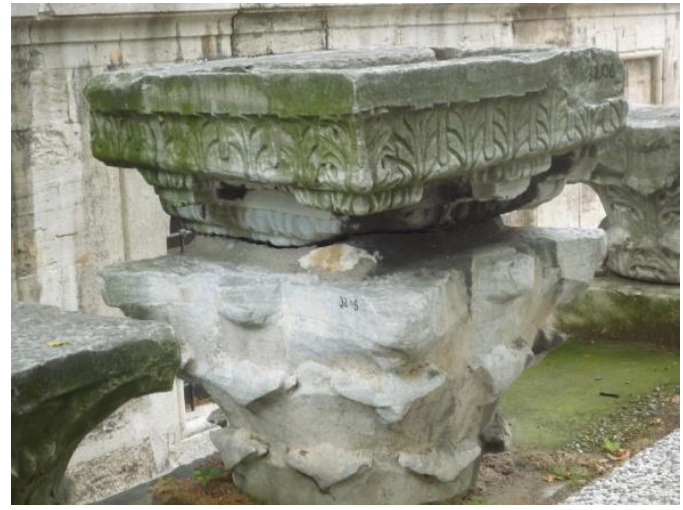

FIG. 26 - Istanbul, Museo Archeologico: frammento di cornice dalla chiesa di San Giovanni Battista all'Hebdomon (inv. 3206).

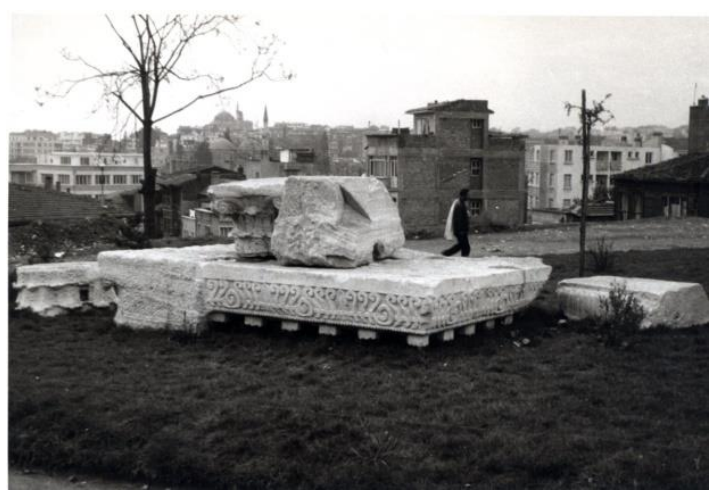

FIG. 28 - Istanbul, Atatürk Bulvarı: frammento di cornice ( Neg. DAI Istanbul).

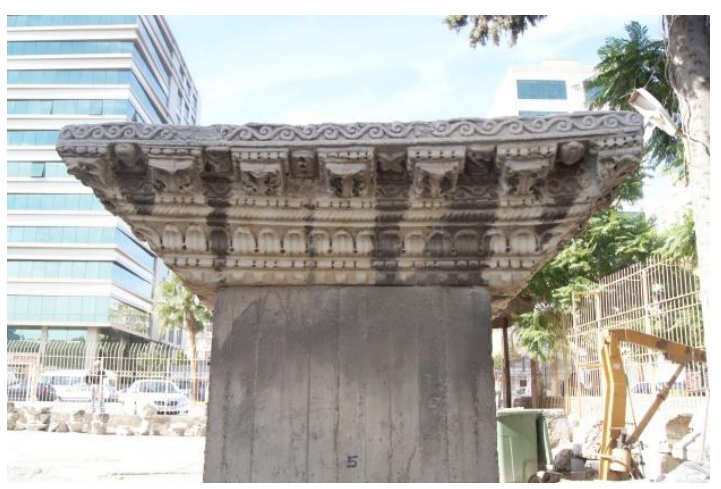

FIG. 30 - Adana, Museo Archeologico: architrave di porta dalla basilica cupolata di Meriamlik. 


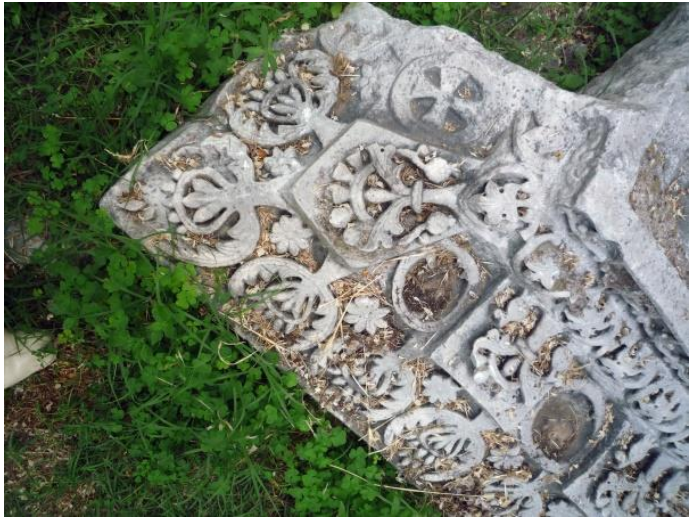

FIG. 31 - Istanbul, Museo Archeologico, deposito: frammento di architrave di porta da San Polieucto.

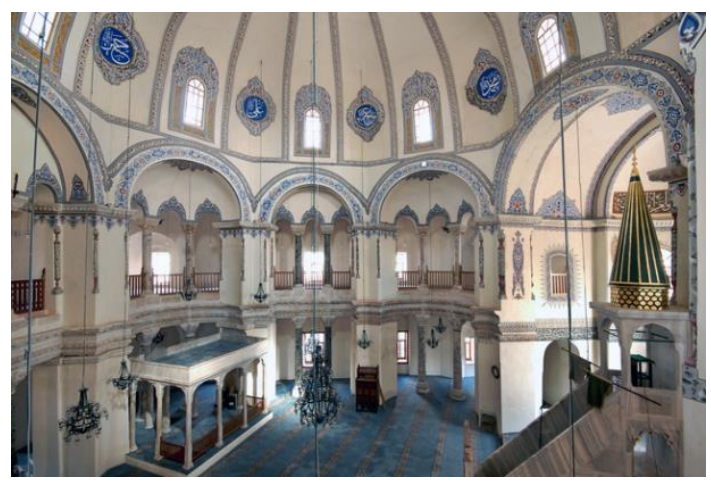

FIG. 33 - Istanbul, Santi Sergio e Bacco: interno.

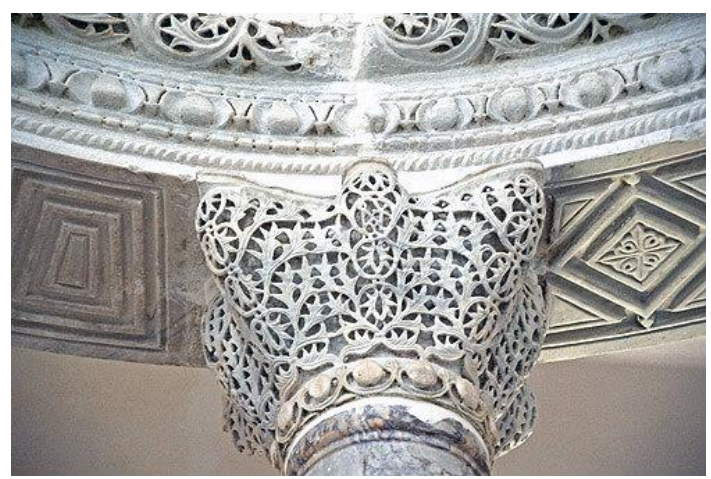

FIG. 35 - Istanbul, Santi Sergio e Bacco: capitello polilobato.

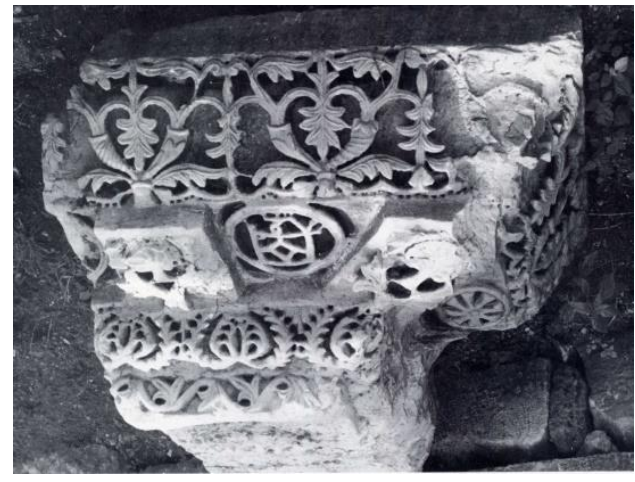

FIG. 32 - Istanbul, Museo Archeologico, deposito: frammento di architrave di porta da San Polieucto (da Harrison 1966).

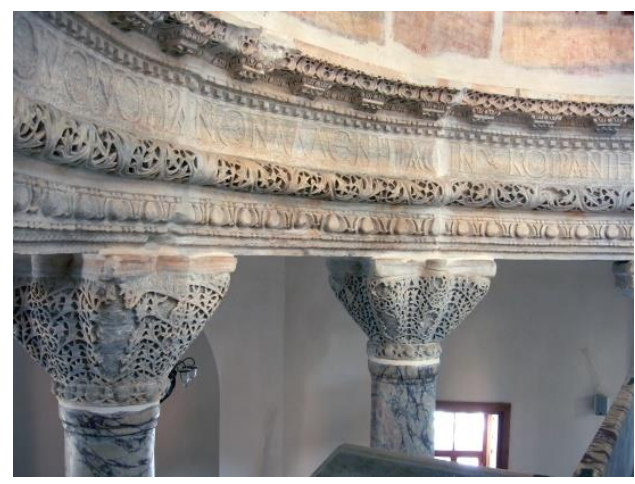

FIG. 34 - Istanbul, Santi Sergio e Bacco: trabeazione e capitelli polilobati.

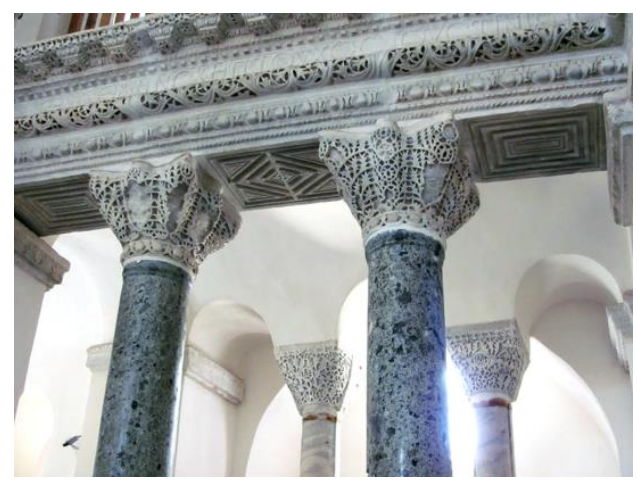

FIG. 36 - Istanbul, Santi Sergio e Bacco: capitelli polilobati e capitelli imposta in opera nella trifora meridionale. 


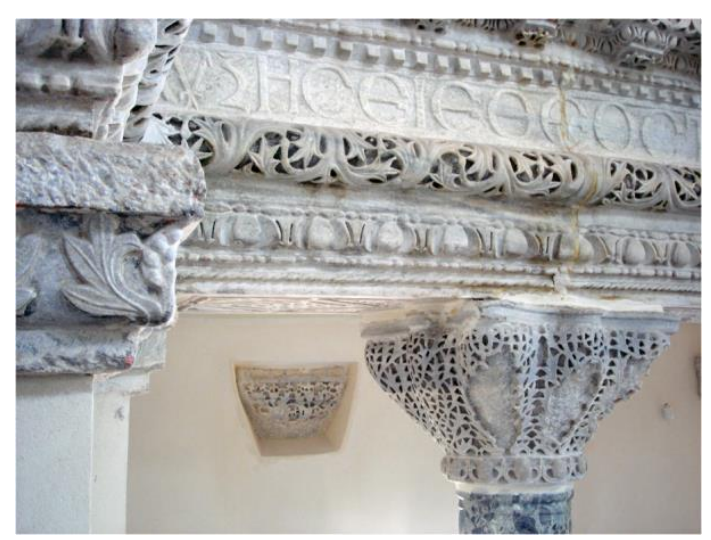

FIG. 37 - Istanbul, Santi Sergio e Bacco: capitello imposta della trifora settentrionale.
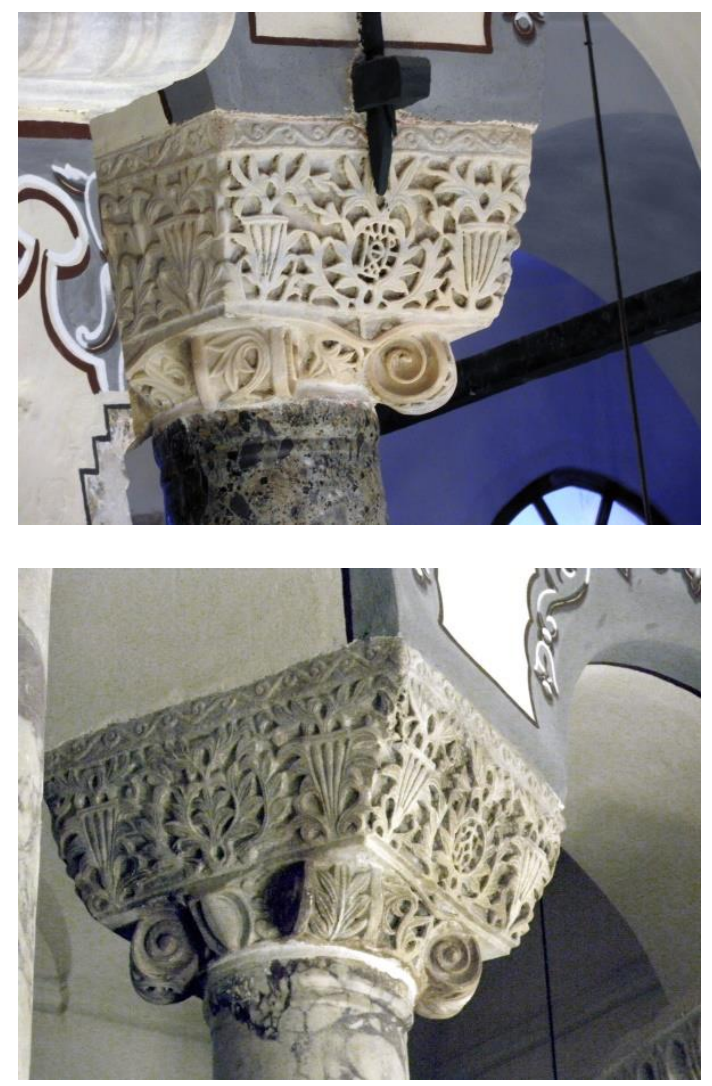

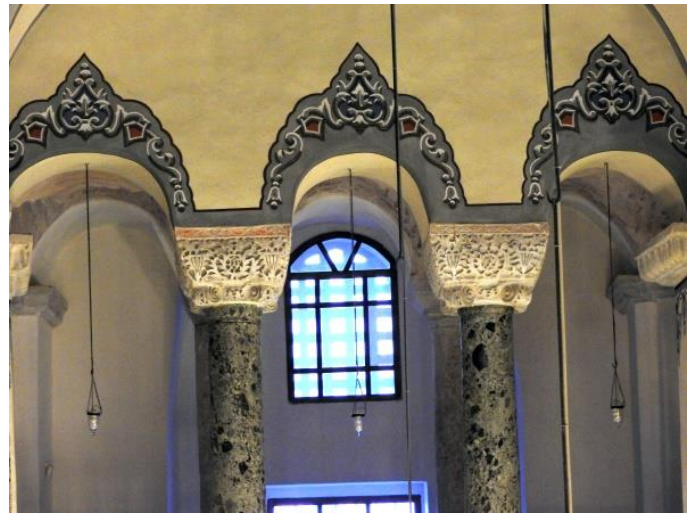

FIG. 38 - Istanbul, Santi Sergio e Bacco: capitelli ionici ad imposta nella galleria.

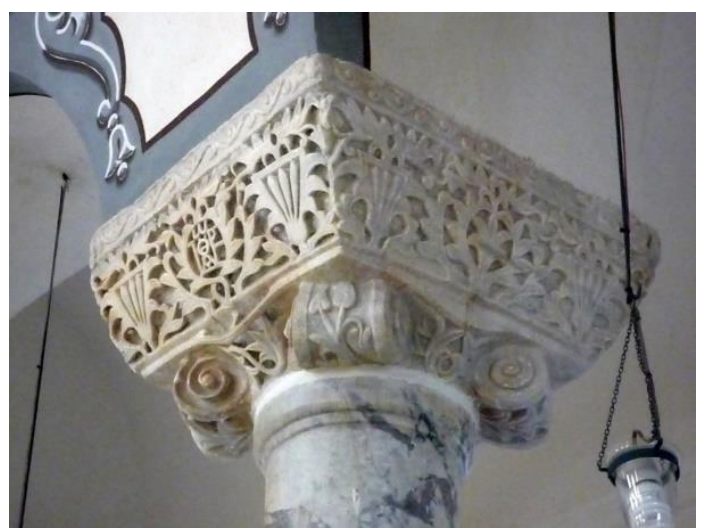

FIG. 39a-b-c - Istanbul, Santi Sergio e Bacco: capitelli ionici ad imposta nella galleria. 


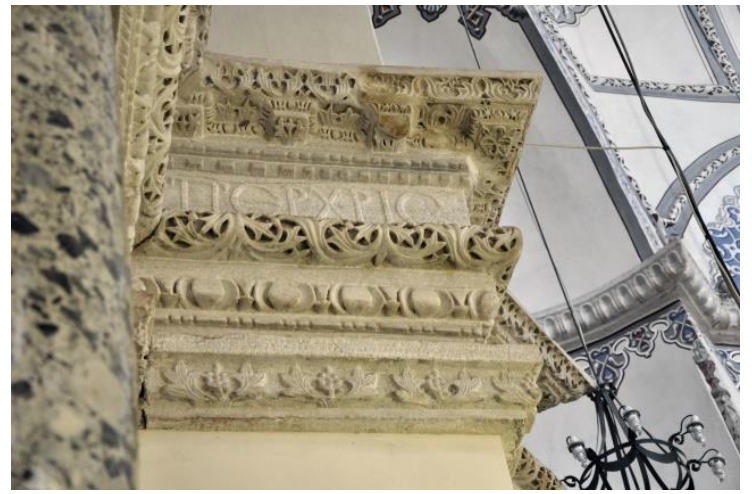

FIG. 40 - Istanbul, Santi Sergio e Bacco: trabeazione, restauro.

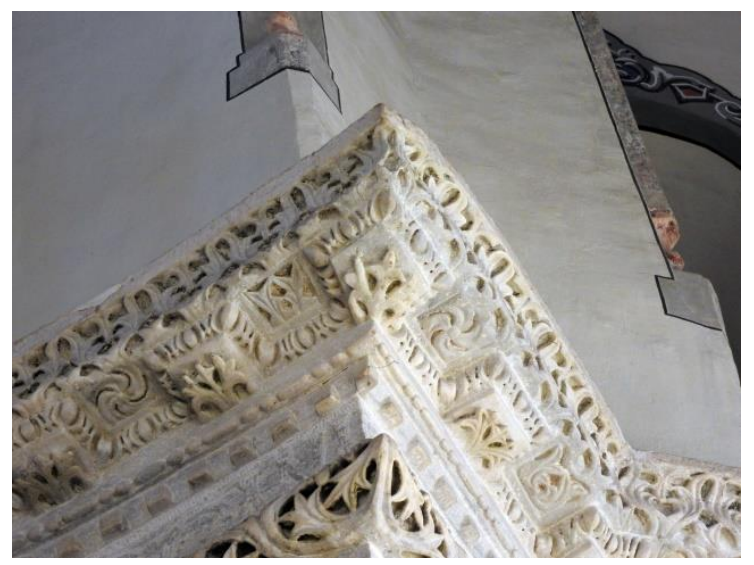

FIG. 42 - Istanbul, Santi Sergio e Bacco: trabeazione, mensola diagonale.

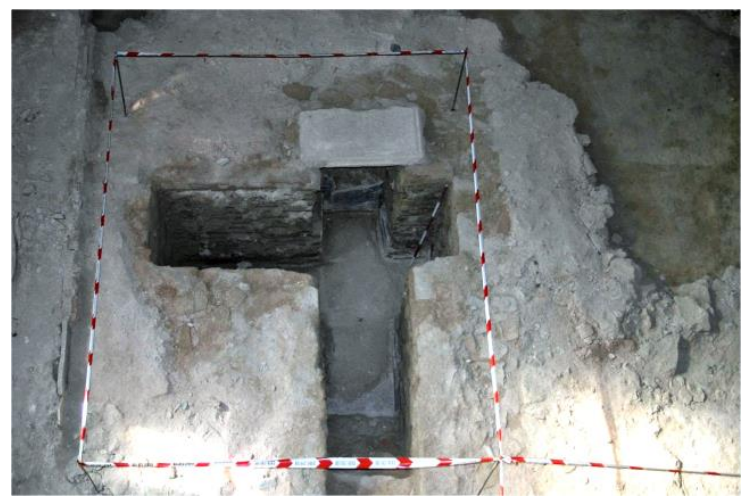

FIG. 44 - Istanbul, Santi Sergio e Bacco: trabeazione, cripta.

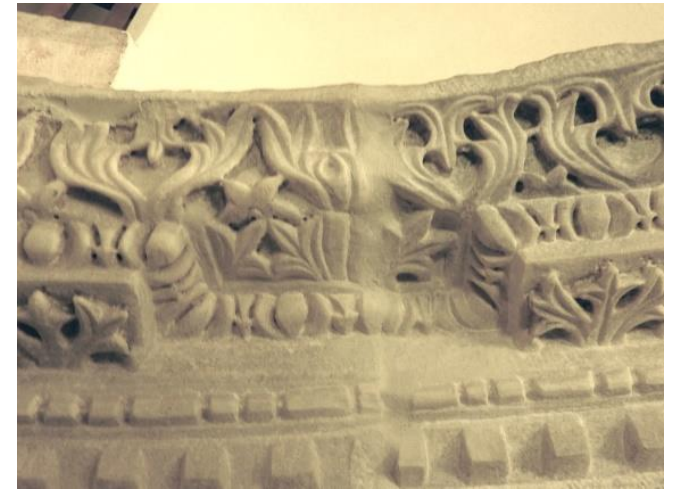

FIG. 41 - Istanbul, Santi Sergio e Bacco: trabeazione, giuntura.

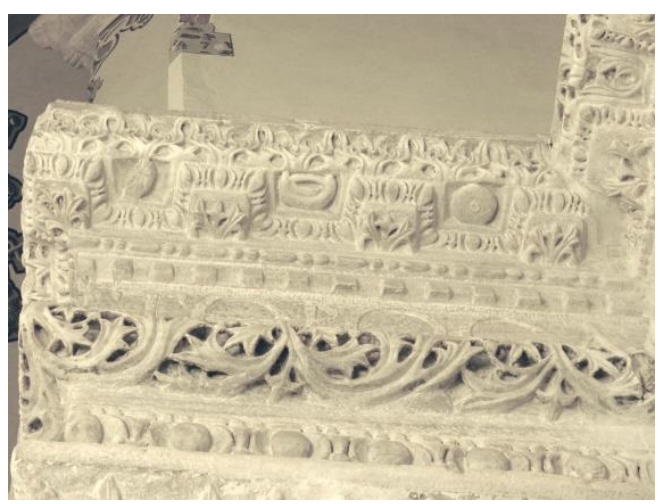

FIG. 43 - Istanbul, Santi Sergio e Bacco: trabeazione, inserti.

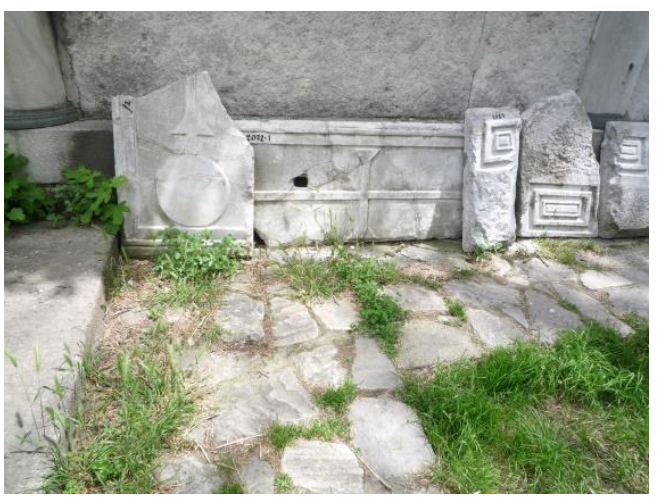

FIG. 45 - Istanbul, deposito del Vakiflar: lastra frammentaria dal pavimento. 


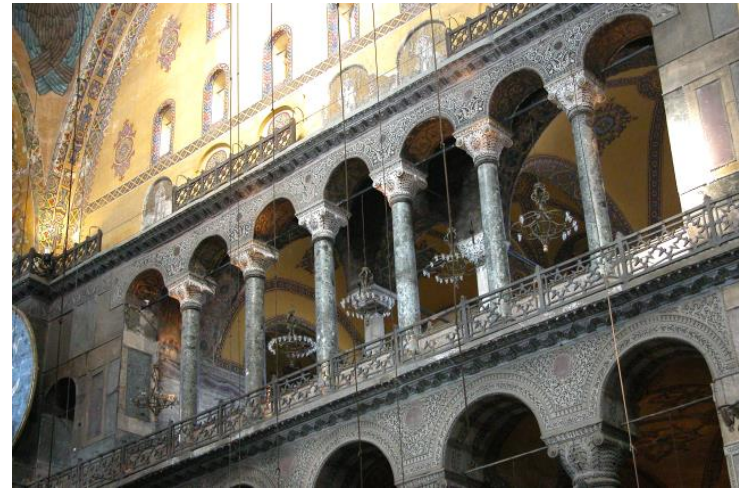

FIG. 46 - Istanbul, Santa Sofia /: tiimpano nord.

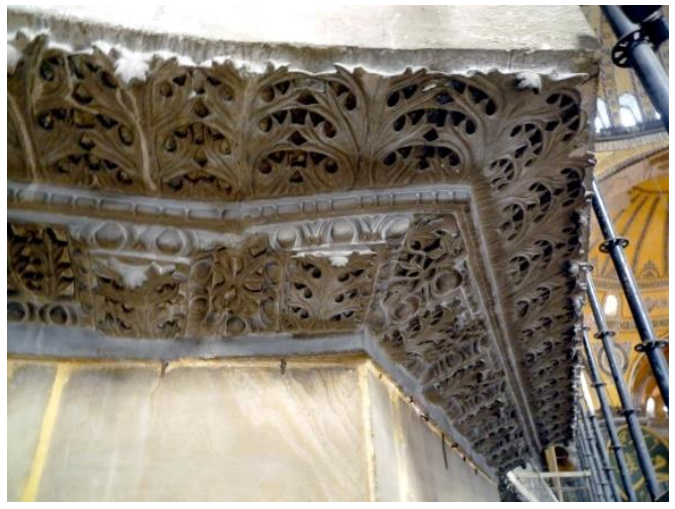

FIG. 48 - Istanbul, Santa Sofia: cornice a modiglioni, $1^{\circ}$ livello, timpano nord.

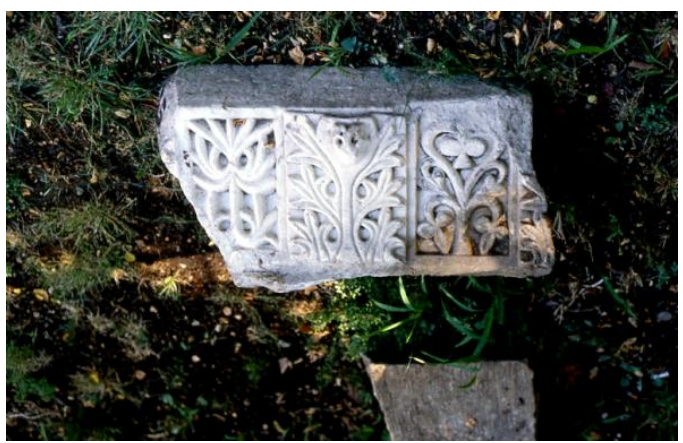

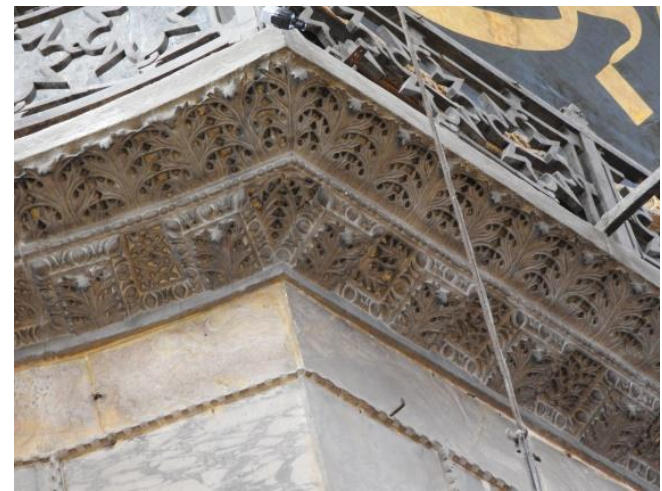

FIG. 47 - Istanbul, Santa Sofia: cornice del pilastro sud-est.

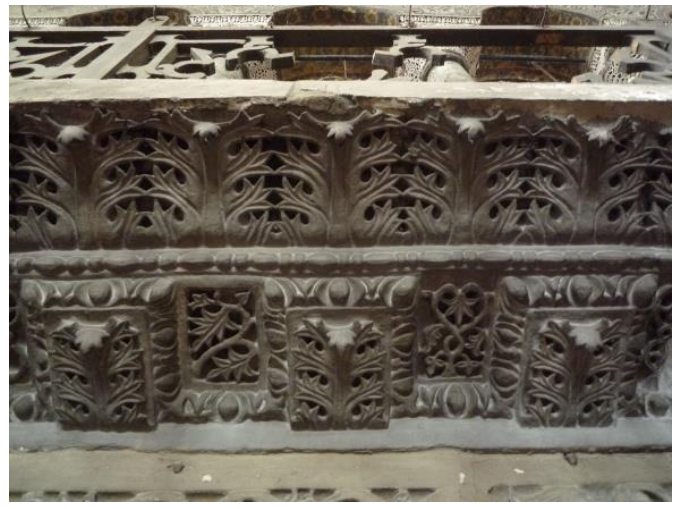

FIG. 49 - Istanbul, Santa Sofia: cornice a modiglioni, $1^{\circ}$ livello, timpano nord.

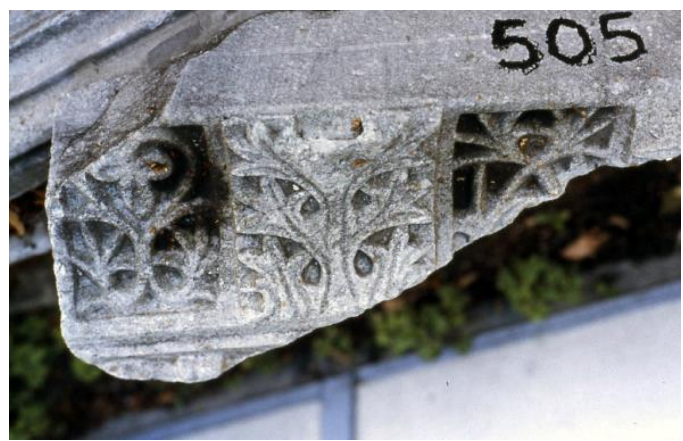

FIG. 50a-b - Istanbul, Ayasofya Muzesi: frammenti di cornice dalla basilica A di Beyazit. 


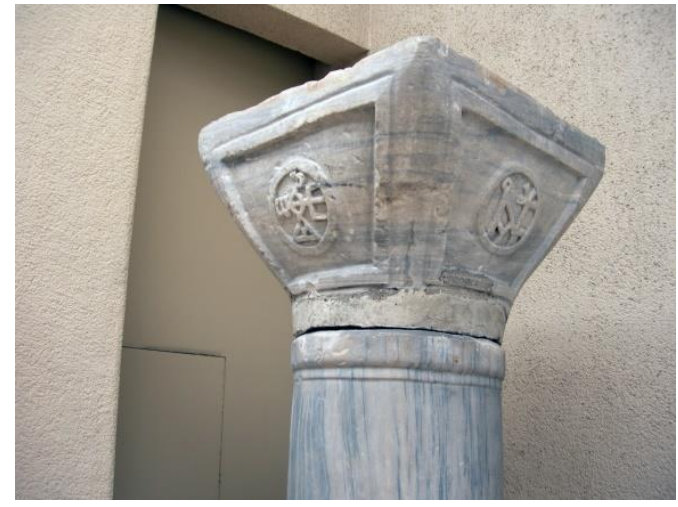

FIG. 51 - Istanbul, museo Archeologico: capitello imposta con monogrammi di Giustiniano e Teodora.

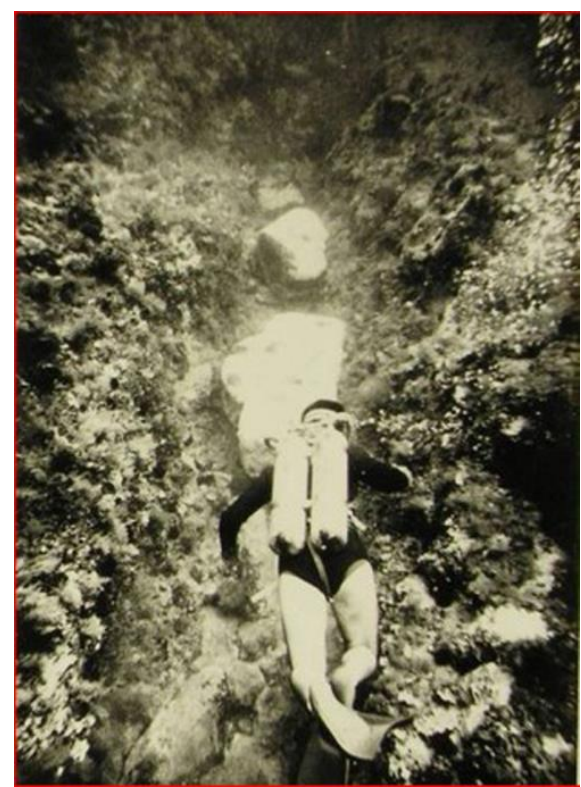

FIG. 53 - Foto di scavo del relitto di Marzamemi (da Kapitän).

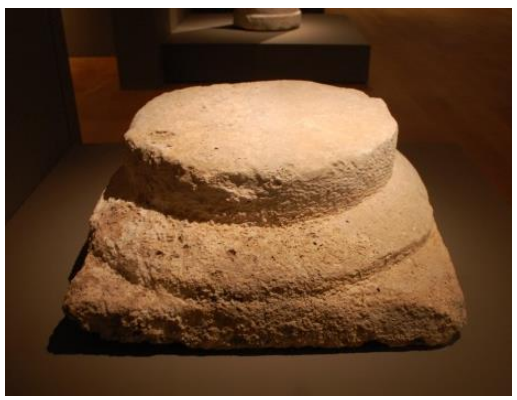

FIG. 55 - Base di colonna dal relitto di Marzamemi.

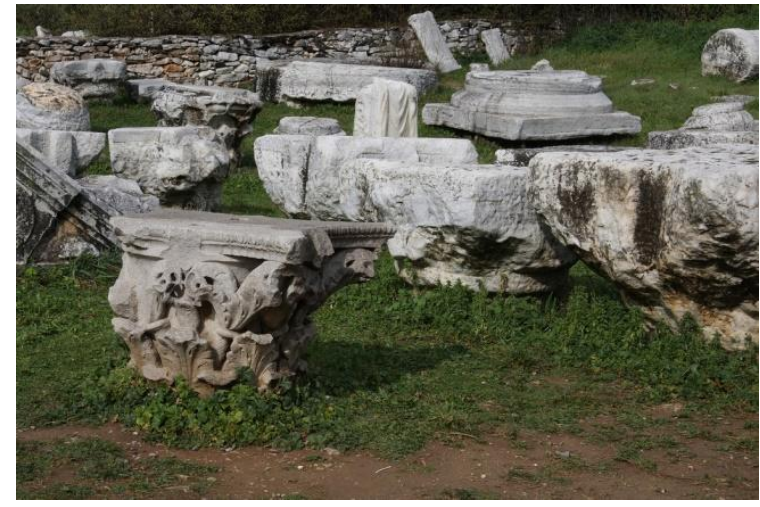

FIG. 52 - Marmara Adas1 (isola di Proconneso), deposito di Saraylar.

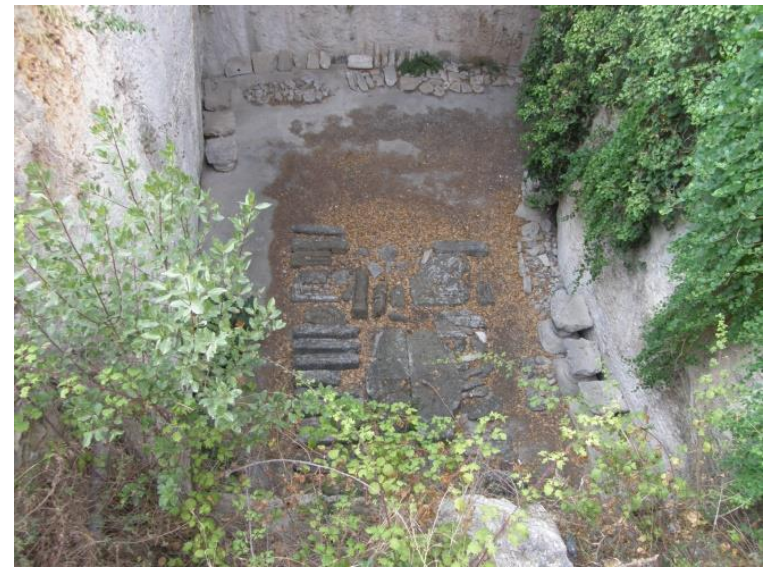

FIG. 54 - Siracusa, Latomie del Paradiso, veduta generale dei marmi del relitto di Marzamemi.

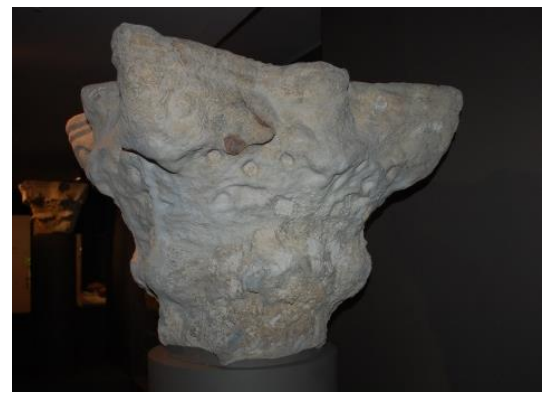

FIG. 56 - Capitello corinzio tipo VII dal relitto di Marzamemi. 


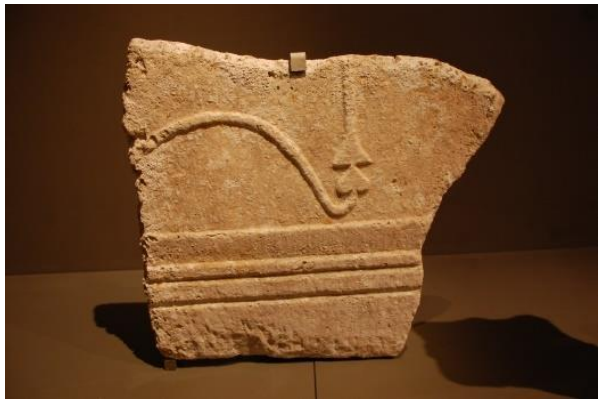

FIG. 57 - Lastra di recinzione frammentaria dal relitto di Marzamemi.

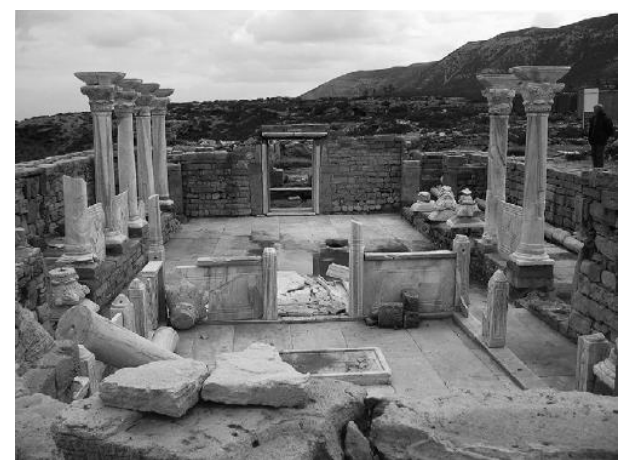

FIG. 59 - Latrun, chiesa occidentale veduta generale dall'abside.

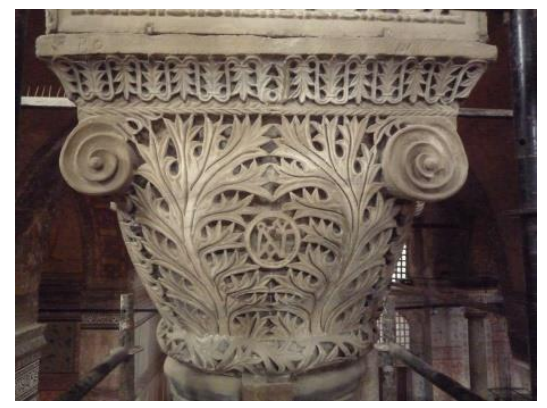

FIG. 61 - Istanbul, Santa Sofia: capitello del lato nord del naos.

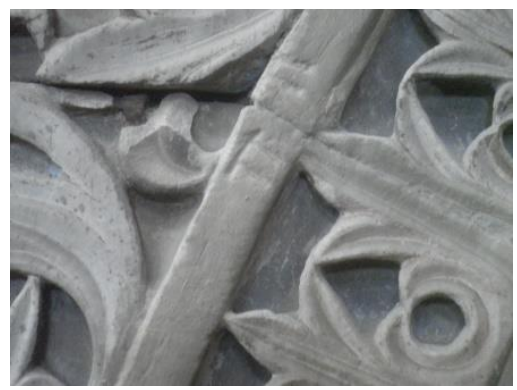

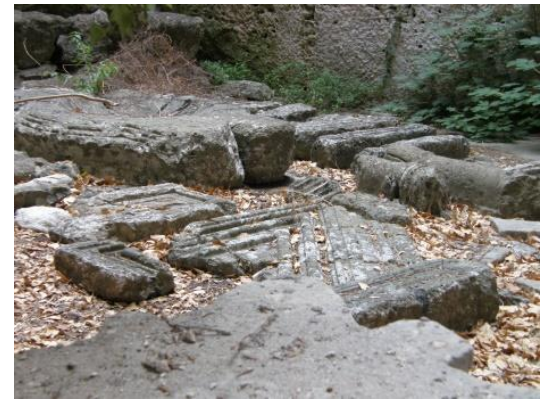

FIG. 58 - Siracusa, Latomie del Paradiso, elementi dell'ambone del relitto di Marzamemi.

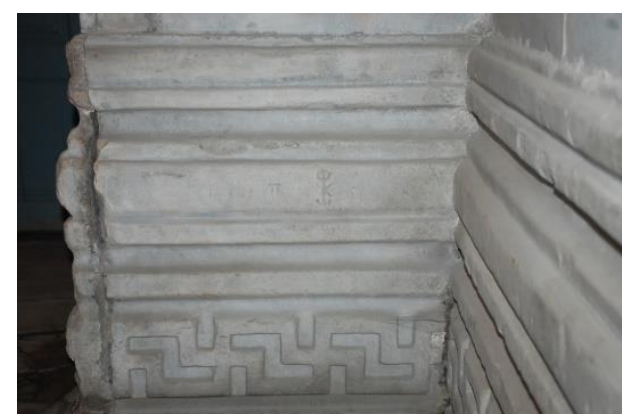

FIG. 60 - Istanbul, Santa Sofia: zoccolo in marmo dell'angolo sudest del naos, part.

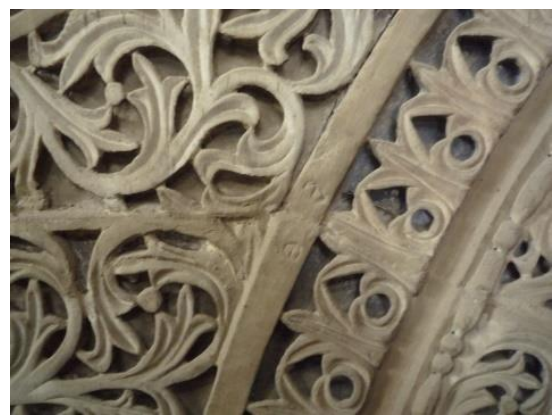

FIG. 62 - Istanbul, Santa Sofia: arcate della parete nord del naos, part, con sigle di assemblaggio $\Theta$.

FIG. 63 - Istanbul, Santa Sofia: arcate della parete nord del naos, part, con sigle di assemblaggio П. 